\title{
Evaluation of Shared Space to Reduce Traffic Congestion: A case study on West Virginia University's Downtown Campus
}

Colin Frosch

Follow this and additional works at: https://researchrepository.wvu.edu/etd

\section{Recommended Citation}

Frosch, Colin, "Evaluation of Shared Space to Reduce Traffic Congestion: A case study on West Virginia University's Downtown Campus" (2017). Graduate Theses, Dissertations, and Problem Reports. 5627. https://researchrepository.wvu.edu/etd/5627

This Thesis is protected by copyright and/or related rights. It has been brought to you by the The Research Repository @ WVU with permission from the rights-holder(s). You are free to use this Thesis in any way that is permitted by the copyright and related rights legislation that applies to your use. For other uses you must obtain permission from the rights-holder(s) directly, unless additional rights are indicated by a Creative Commons license in the record and/ or on the work itself. This Thesis has been accepted for inclusion in WVU Graduate Theses, Dissertations, and Problem Reports collection by an authorized administrator of The Research Repository @ WVU. For more information, please contact researchrepository@mail.wvu.edu. 
Evaluation of Shared Space to Reduce Traffic Congestion: A case study on West Virginia University's Downtown Campus

\title{
Colin Frosch
}

\author{
Thesis submitted to the \\ Benjamin M. Statler College of Engineering and Mineral Resources \\ at West Virginia University \\ in partial fulfillment of the requirements for the degree of \\ Master of Science in \\ Civil Engineering
}

\author{
David Martinelli, Ph.D., Chair \\ Avinash Unnikrishnan, Ph.D. \\ Ronald Eck, Ph.D. \\ Department of Civil and Environmental Engineering
}

Morgantown, West Virginia

2017

Keywords: Shared Space, PTV Vissim, Simulation, Transportation, Traffic Congestion, Alternative Traffic Design, Traffic Calming

Copyright 2017 Colin Frosch 


\section{ABSTRACT \\ Evaluation of Shared Space to Reduce Traffic Congestion: A case study on West Virginia University's Downtown Campus}

\section{Colin Frosch}

Pedestrian and vehicle interactions often lead to conflicts that bring about safety, traffic congestion, and priority or right of way issues. Common methods used in the past to combat said issues have largely relied on the principle of separating the motions of pedestrians and vehicles by means of bridges, tunnels, signals, and access restrictions. A different approach known as share space aims to solve the same problems with a less structured and defined environment which instead places more reliance on human interaction and perception. Although it has been used in multiple scenarios across Europe with success, instances of shared spaces in the United States are few. In the past, the success of shared space has mainly focused on safety, aesthetic, and pedestrian use metrics, with little quantitative knowledge regarding the traffic congestion relief benefits. This research focuses on evaluating and quantifying the traffic congestion relief abilities of shared space designs utilizing Vissim traffic microsimulation software, and the economic impact these changes can make. A major pedestrian crossing location on West Virginia University's Downtown campus along a major urban arterial was chosen as the case location upon which the model was to be built. This location posed unique aspects which made it a prime choice for this research as the major concern for years has been traffic congestion, in addition to pedestrian safety and aesthetic appeal. The results of the research indicated that shared space can reduce the congestion within a specific location, but more importantly will reduce the impact that that congestion zone has on the greater network. In the case of the selected location, the resulting benefits overcame the cost of implementing the proposed design within 10 years. 


\section{ACKNOWLEDGEMENTS}

Without the help, love, and support of many people, this thesis and accompanying master's degree would not have been possible. I would like to take the time to thank the following people in particular:

To Gina, my best friend and true love, for her unwavering support and love throughout my time at WVU, her persistence to motivate me, and for continuing to make me smile.

To my parents, Ken and Julie, for the many hours teaching me as a child, forming me in to the man I am today, and for always being there to support me in all of my endeavors.

To my siblings, Landon, Erin, Amy, Sierra, and Christina, for helping me always find the joy in life and learning how to work in a team and be a leader from day one.

To my professors and committee members, Dr. Avinash Unnikrishnan, Dr. David Martinelli, and Dr. Ronald Eck, for going above and beyond to supply students like me at WVU with the best opportunities to succeed.

To my G103 office mates, Ryan Sigler, Cate Schlobohm, Brandon Diaz, and Gina Frosch, for helping me to grow as an ambassador, having me join you as a GA and supporting me while I pursued this degree, and for providing me with many fond and happy memories over the last 5 years.

To West Virginia University, and the Statler College, for providing my fellow students and I with endless opportunities to pursue our dreams.

To David Palley and Andrew Poszich, my master's thesis support group.

To Mark Schuchardt, Ethan Garrison, Matthew Palmer, Daniel Knight, Elliot Hair, and all of the friends I made while at WVU for the long nights studying, group projects, and fun times outside of the classroom. 
To Joe Patten, the WVU administration, and Stantec for working on this project with my team and I in the hopes of improving traffic and safety in Morgantown, WV.

To Andrew Morgan and the LTAP center for loaning us the data collection equipment

To Ryan Campione and Tommy Casto for representing the WVU Student Government Association and helping to get this project started.

To Elijah Meyer and David Donaldson for assisting in collecting data, reviewing the literature, and presenting on our findings.

To Cate Schlobohm, Michelle Poland, Sonja Wilson, and each of their staffs for assisting in the gathering of volunteers for the data collection.

To Alexander Pannaccione, Gina Paugh, Samantha Witsberger, John Guice, Ian Cook, Julia Hatgelakas, Ryan Butler, Ryan Renzi, Sophia Chico, Matthew Palmer, Tori Smith, Derek Hamman, Henry Loh, Wyatt Trevithick, Anika Coolbaugh, Katie Warner, Ryan Campione, Kevin McCabe, Courtney Bee, Paul Sibrell, Trever Ramundo, Tommy Folino, Stephen Han, Ali Kowalski, Alexis Blevins, Josh Watson, Marwa Ataya, and Becca Cokeley for volunteering their time to assist in the collection of traffic data at the case study location.

To the Dwight D. Eisenhower Transportation Fellowship Program for funding and supporting my graduate research in pursuit of this thesis.

To everyone else who I may not have mentioned above, thank you for your friendship and help in whatever way it may have been to make this possible. 


\section{TABLE OF CONTENTS}

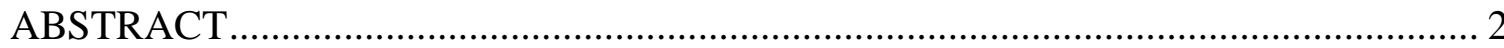

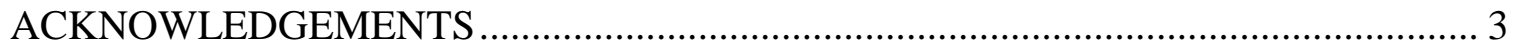

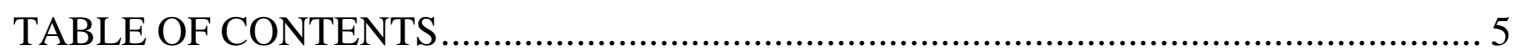

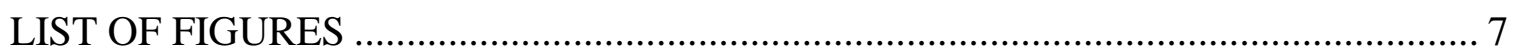

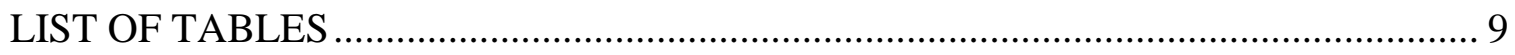

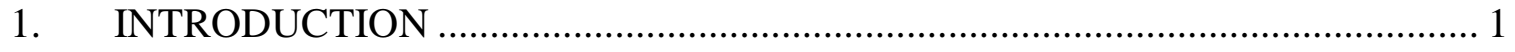

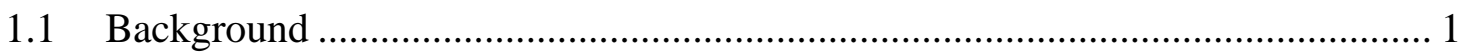

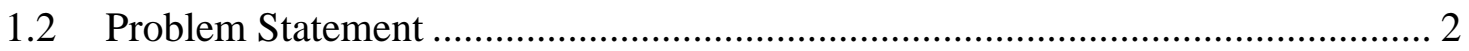

1.3 Research Objectives and Questions .......................................................... 2

1.4 Scope

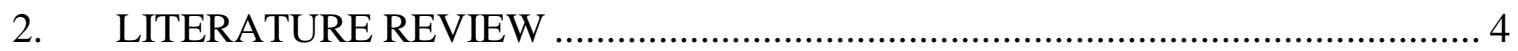

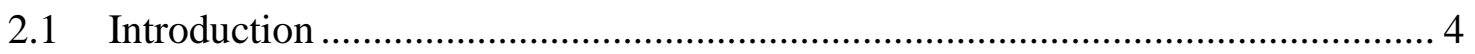

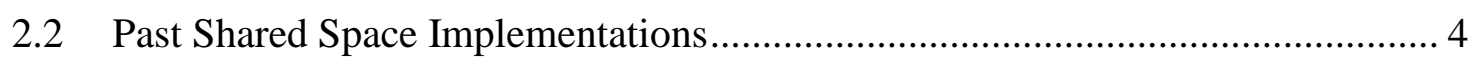

2.2.1 European Town and Village Shared Spaces ................................................ 6

2.2.2 Other European Shared Spaces …………………..................................... 8

2.2.3 Shared Space Outside of Europe ……………………………………….... 11

2.3 Shared Space Safety Research ..................................................................... 15

2.3.1 Risk Assessment principles.................................................................... 16

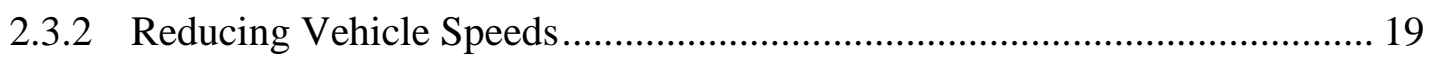

2.3.3 Reducing Conflicts between Pedestrians and Vehicles................................. 21

2.4 Economic, Social, and Traffic Benefits ....................................................... 23

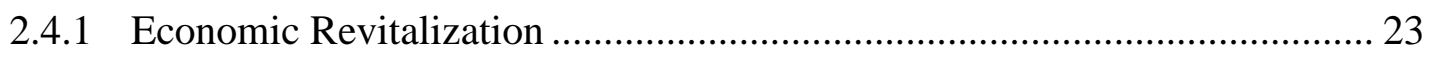

2.4.2 Public Perception........................................................................... 25

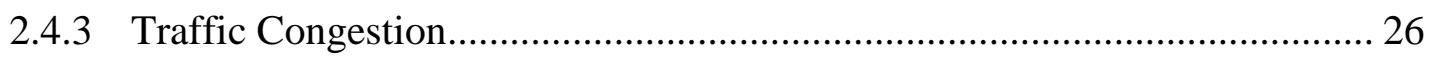

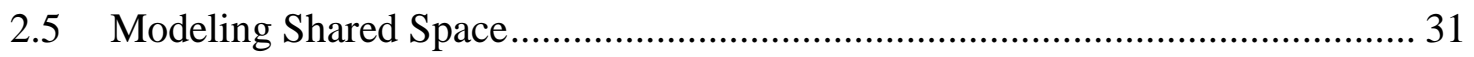

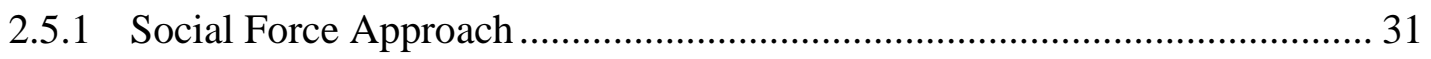

2.5.2 Viswalk and Vissim combination ........................................................... 36

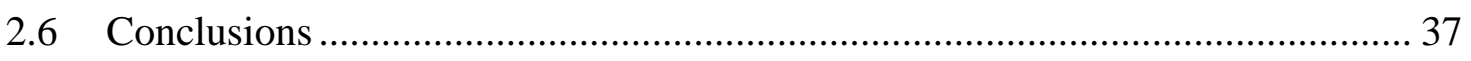

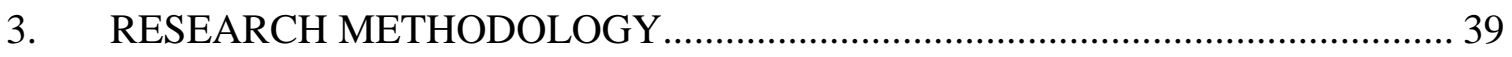

3.1 Creating the PTV Vissim Model.................................................................... 39

3.1.1 Adapting PTV Vissim to Shared Space ...................................................... 40

3.1.2 Designating and Spacing Pedestrian Routes .............................................. 42

3.1.3 Additional shared space modeling assumptions............................................. 44

3.2 Case Study Simulation ........................................................................... 44 
3.2.1 Fitting the Model to the Study Location ………………............................... 51

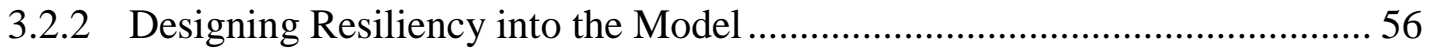

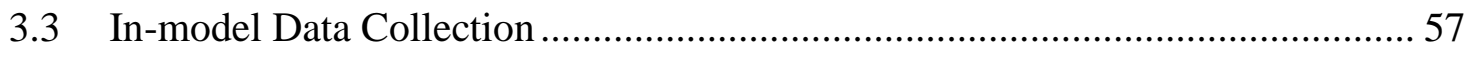

3.3.1 Assessing Congestion Relief.................................................................. 58

3.3.2 Measuring Economic Benefits ................................................................ 59

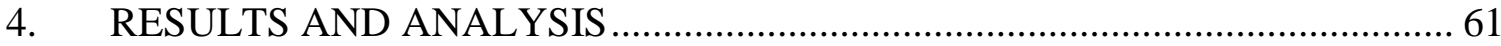

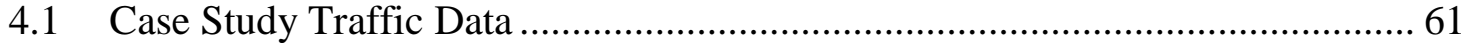

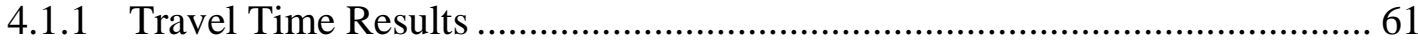

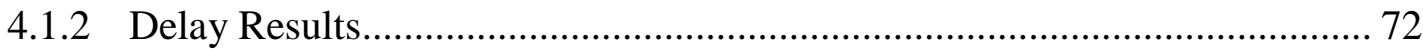

4.1.3 Queue Length Results .......................................................................... 80

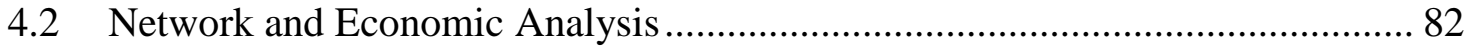

4.2.1 Net Present Value and Payback Period ........................................................... 85

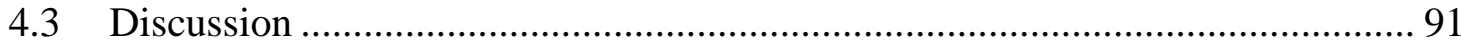

5. CONCLUSIONS AND RECOMMENDATIONS …………................................ 92

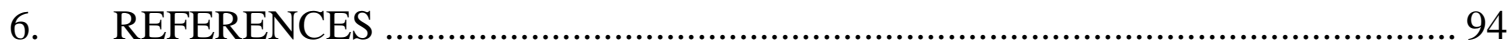

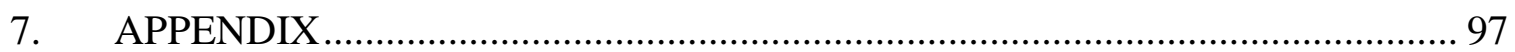




\section{LIST OF FIGURES}

Figure 1: Traditional Separation versus shared space public realm (Hamilton-Baillie 2008)....... 5

Figure 2: Shared Space in Bohmte, Germany (Hall 2008) ..................................................... 7

Figure 3: Poynton, England before and after shared space implementation (Wilkinson 2014) ..... 8

Figure 4: Sonnenfelsplatz - Graz, Austria shared space (Fischer 2011) ................................. 10

Figure 5: Noordlaren Shared Space in front of primary school (Allianz 2012) ........................ 11

Figure 6: Shared Space on Bell Street in Seattle Washington (Behrens 2014) ......................... 13

Figure 7: Mean Speed at each 15 minute period, by Site (Shore 2010) .................................... 20

Figure 8: Proportion of users giving way based on average speed (Shore 2010) ...................... 28

Figure 9: Proportion of users giving way based on total 15 minute vehicle flow (Shore 2010) .. 29

Figure 10: Proportion of users giving way based on total pedestrian count (Shore 2010) .......... 30

Figure 11: Observed vehicle, bicyclist, and pedestrian paths in Graz, Austria (Rudloff 2013) ... 42

Figure 12: Current Grumbein's Island Configuration on University Avenue in Morgantown, WV

(Google) ................................................................................................ 45

Figure 13: Data Collection Devices (Left: manual turning counter, Right: mounted radar vehicle

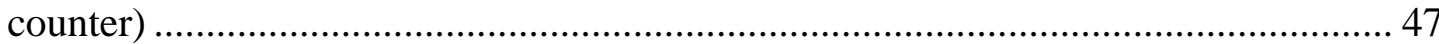

Figure 14: Radar and Manual Counter Data Collection Locations (Google Maps) ................... 47

Figure 15: Morning One-Hour Peak Vehicular Volume at Case Study Location ...................... 48

Figure 16: Evening Peak Vehicular Volume at Case Study Location ....................................... 49

Figure 17: Pedestrian Crossing Volume During Non-Peak 15 Minute Increment ..................... 49

Figure 18: Pedestrian Crossing Volume During Peak 15 Minute Increment ............................ 50

Figure 19: Pedestrian Crossing Volume per Minute on University Avenue over One Hour Time

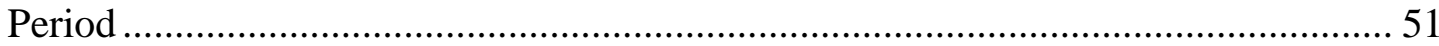

Figure 20: Forecasted Pedestrian O-D Pairs (Blue: Origin/Destination, Yellow: Routes, Red:

Shared Space Boundary) (Google) ...................................................................... 52

Figure 21: Shared Space Conceptual Design (Stantec) ........................................................ 54

Figure 22: Pedestrian Crossing and Vehicular Links in PTV Vissim Simulation ....................... 55

Figure 23: Designated Conflict Areas in PTV Vissim Simulation .......................................... 56

Figure 24: Illustration of Queue Length Measurements beginning at Grumbein's Island ........... 59

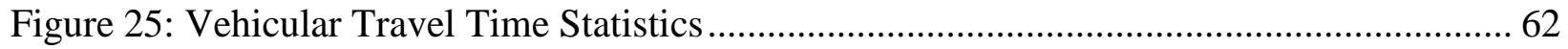

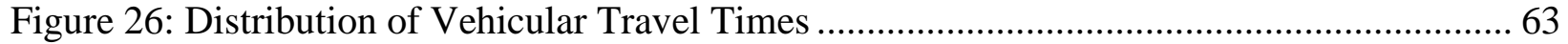

Figure 27: Vehicular Travel Time Statistics During Non-Peak Pedestrian Time Period............ 65

Figure 28: Vehicular Travel Time Statistics During Peak Pedestrian Time Period .................... 66

Figure 29: Distribution of Vehicular Travel Times Observed During the Non-Peak Pedestrian

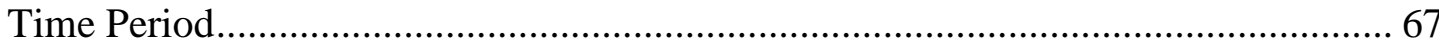

Figure 30: Distribution of Vehicular Travel Times Observed During the Peak Pedestrian Time

Period

Figure 31: Vehicular Flow during Peak Pedestrian Volume Period of 15 Minutes ................... 68

Figure 32: Vehicular Travel Time Statistics split in SB and NB Directions ............................ 69

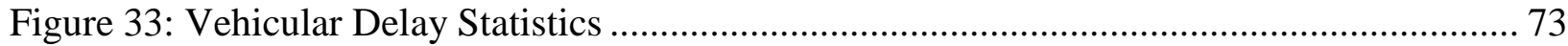

Figure 34: Distribution of Vehicular Delay ................................................................. 75

Figure 35: Vehicular Delay Statistics Split Between Peak and Non-Peak Pedestrian Time Periods

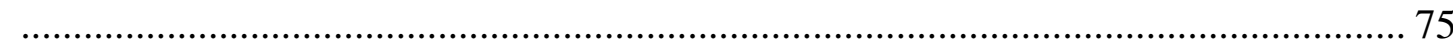

Figure 36: Distribution of Vehicular Delay during the Peak Pedestrian Volume Period............ 77 
Figure 37: Distribution of Vehicular Delay during the Non-Peak Pedestrian Volume Period..... 77

Figure 38: Vehicular Delay Statistics Split Between Northbound (NB) and Southbound (SB) Directions

Figure 39: Maximum Queue Length in 5 Minute Intervals Measured for Vehicles Traveling in the Southbound Direction

Figure 40: Maximum Queue Length in 5 Minute Intervals Measured for Vehicles Traveling in the Northbound Direction

Figure 41: Vehicular Travel Time and Delay Statistics for Vehicles Traveling in the Southbound Direction from Stewart Street to 300 feet Past the Intersection 83

Figure 42: Vehicular Travel Time and Delay Statistics for Vehicles Traveling in the Northbound Direction from North High Street to 300 feet Past the Intersection 84

Figure 43: Queue Length 300' Away from Neighboring Intersections Along University Avenue

Figure 44: Pedestrian Crossing and Vehicle Travel Links in PTV Vissim Model of Current State 


\section{LIST OF TABLES}

Table 1: Pedestrian Data Collection 2015 Summary Values..................................................... 50

Table 2: PERT Expected Travel Times for Each Model in Seconds......................................... 71

Table 3: PERT Expected Delay Calculated Values for all Seven Models ................................ 80

Table 4: Total Money Saved per Year and Payback Period Financial Calculation for Primary

Shared Space Model and Average of all Six Shared Space Models ........................... 88

Table 5: NPV and IRR Analysis of Shared Space Primary Model........................................... 89

Table 6: NPV and IRR Analysis of Shared Space Models Average Across All Six Models...... 90

Table 7: Pedestrian Volumes Used in model of Current Traffic ................................................ 97

Table 8: Pedestrian Volumes Used in Model of Shared Space ................................................ 98 


\section{INTRODUCTION}

\subsection{Background}

Shared space traffic designs have been used in an increasing number of countries around the world to solve congestion, safety, accessibility, and community issues. This design concept was first pioneered in the Netherlands by Hans Monderman in the late 1900s, but has since been adapted to fit other case-specific areas within western Europe and most recently in North America. The applications of shared space range between urban and suburban locations and have been found most suitable for areas used by multiple modes of transport. Although the specific implementation details of each shared space have varied greatly across time and location, the bottom line principle of ridding an area of most if not all traffic signs, demarcations, and traditional controls to allow multi-modal users more independence and less decision making reliance on these engineered elements have been present.

By forcing drivers and pedestrians to no longer rely on traffic signals, signs, and designated crosswalks to create distinct rules of priority, they must instead be more alert to their surroundings and communicate with other users. Users quickly realize this upon entering a shared space environment and begin to proceed with more caution and make more eye contact between users. These actions by the individual user have been scientifically shown to increase the pedestrian safety and decrease the average vehicle speeds. It has also been noted anecdotally multiple times that the congestion and travel time through a specified area has been reduced as result of a shared space being implemented, however the data to back up these claims is rare. Finally, shared space designs have been shown to greatly increase the public's perception of an intersection, corridor, or locale through the use of improved aesthetic elements. These now more 
welcoming areas have also in turn experienced improved and revitalized economic markets brought about by an increase in mostly bicycle and pedestrian users to the area.

\subsection{Problem Statement}

As mentioned above, there is an untapped potential with shared space to address congestion and traffic delay issues that arise at multi-modal intersections or corridors. The traffic flow dynamics present based on user behavior within a shared space have the potential to reduce this congestion and delay problem. Municipalities and design firms have unfortunately not turned to shared space yet as a main way to address congestion problems. A simple, yet effective, way to examine the traffic efficiency effects of an implemented shared space design at a case location is necessary in this industry to demonstrate them to engineers, planners, government officials, and community members.

\subsection{Research Objectives and Questions}

The objective of this research was to create a simple way for industry professionals to evaluate the traffic congestion and vehicular delay impacts provided by a shared space design alternative compared to a traditional design. The research sought to develop a microsimulation traffic modelling method using existing tools which would provide sufficient information to be used in decision making processes based on metrics such as travel time, delay, and the resulting economic benefits.

\subsection{Scope}

The objective model was created in PTV Vissim software readily available on the market to be utilized by traffic engineers and planners to create microsimulations. A case study location known as Grumbein's island, a major pedestrian crossing on the West Virginia University downtown campus, was chosen for its widely known vehicular congestion and delay problem 
stemming from the very cyclical pattern of student pedestrians crossing at a single location. Vehicular and pedestrian traffic volume as well as turning movement percentages was collected in order to create a realistic model in PTV Vissim. In addition to the data collected, satellite images of the current roadway design and a conceptual design drawing were used as a baseline for the PTV Vissim model. This model would then be used to extract travel time, delay, and other such traffic information in order to assess the impact a shared space design would have. This information combined with a construction and design cost estimate and local cost of living data was then used to estimate the economic benefits of implanting this proposed design. 


\section{LITERATURE REVIEW}

\subsection{Introduction}

To set the premise of the research study, a review of previously implemented shared spaces and their common components was completed. Since various types of shared spaces have been implemented in a variety of locations around the world in recent years, researchers have been provided the opportunity to study many aspects of them in actuality. The benefits received in areas with shared space have been able to be quantified to promote future uses of the infrastructure, with the exception of congestion relief. This missing component of shared space, as well as current methodologies to simulate shared space, will provide the basic need for the research conducted in this thesis.

\subsection{Past Shared Space Implementations}

The shared space concept was first implemented by a Dutch traffic engineer, Hans Monderman, in the late $20^{\text {th }}$ century as a way to decrease pedestrian fatalities in the frequent village setting found in the Netherlands. (Hamilton-Baillie 2008) While most other traffic engineers of the time would have increased the amount of traffic control devices such as striping, signals, barriers, and other man made implements to separate the flows of vehicle traffic from that of pedestrians, Monderman proceeded in the completely opposite ideological direction. He began in the towns of Oudehaske and Makkinga by striving to center his design on the principle of making the areas "more like a village". (Hamilton-Baillie 2008) This was accomplished by removing the traditional roadway markings and control devices, and allowing the drivers to make their behavioral decisions through observation of their surroundings. Once these designs were completed and constructed, the data results showed a vehicle speed reduction of $40 \%$, compared 
to the $10 \%$ the engineers were typically observing at locations with conventional traffic calming devices. (Hamilton-Baillie 2008)

Multiple studies have been completed correlating the speed of vehicles to the safety of pedestrians. Statistics provided by Great Britain's Department of Transportation show a significant decrease in pedestrian fatalities as vehicle speeds drop below 30 miles per hour, from a $45 \%$ chance of fatality occurring during a crash down to only $5 \%$ occurrence while the vehicle is traveling at 20 miles per hour. (Gilman 2007) Data from the US General Estimates System database showed that from 1994-1996 not only were fatalities much more infrequent in a slow speed (20 mph or less) crash, there were also many fewer instances of pedestrian involved crashes at that speed level. (Leaf 1999) Therefore, it is not surprising that the locations at which Monderman implemented shared space designs also experienced reductions in "serious accidents". (Hamilton-Baillie 2008) Monderman's theory that at lower speeds pedestrians and drivers would be able to establish eye contact and socially interact to anticipate each other's behavior and determine their own appropriate behavior had worked to reduce accidents and injuries. (Garman 2012)
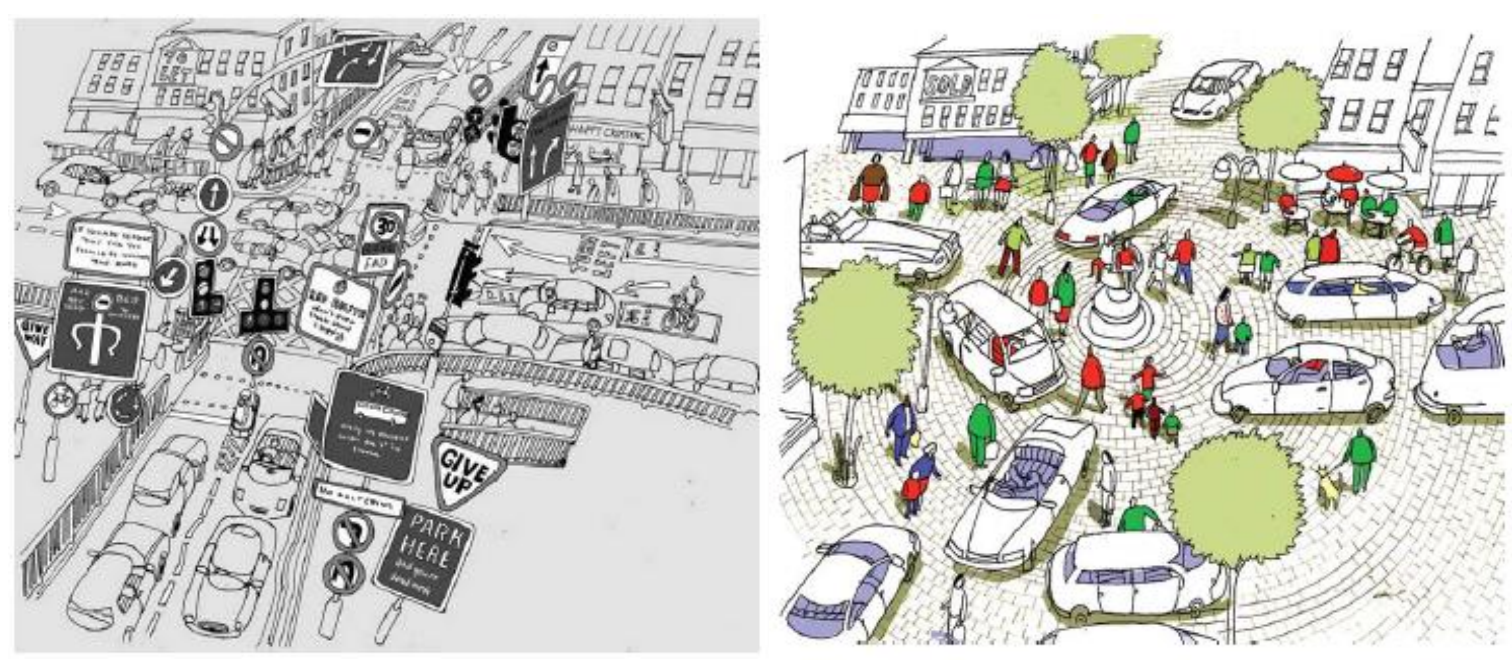

Figure 1: Traditional Separation versus shared space public realm (Hamilton-Baillie 2008) 
With this breakthrough in traffic engineering culture, many shared space proponents such as Ben Hamilton-Baillie began promoting the use of shared space as a new urban design and traffic engineering method. Unlike other advances in transportation engineering which required new technology, engineering processes, or mathematical and scientific advances, shared space was a change in ideology. Ben Hamilton-Baillie, a UK based urban designer points to the street as a public realm to be shared by all of the citizens and users. Prior to the advance of the automobile, public realms encompassed the majority of cities in the means of market squares, parks, and streets. However as driving became more prominent the need to make these streets more standardized became apparent. In the US this meant the introduction of the Manual on Uniform Traffic Control Devices in 1935, (Hawkins 2015) and the introduction of similar policies and reports in the UK like the 1963 Traffic in Towns report. These policies, guidelines, and reports not only encouraged but urged the use of segregation or separation between vehicular traffic and pedestrians. The result was a widespread use of bridges, underpasses, barriers, traffic signals, and a reduced number of pedestrians or cyclists. (Hamilton-Baillie 2008) Shared space however, reverses this clock and returns the streets to again be used as a public realm. Soon after the first true implementation of shared space in the Netherlands by Monderman, shared spaces could be seen in Denmark, Germany, Spain, Sweden, and were beginning to gain traction in places like the UK. (Hamilton-Baillie 2008)

\subsubsection{European Town and Village Shared Spaces}

In Bohmte Germany, a small town of 13,000 people, officials got rid of all of the traffic control devices on their busiest street in 2007 to transform it in to a shared space as can be seen in Figure 2. (Whitlock 2007) Prior to the implementation, citizens were quoted as saying it was 
difficult to cross the street, and took a long amount of time to do so. (James 2008) Pedestrians crossing the main road of Bremen Strasse which served 12,000 vehicles daily on average paired with a high percentage of truck through traffic, it was determined that a shared space would be the most suitable solution. (Whitlock 2007) Within the four weeks following the opening of the shared space sections not a single accident had occurred, where there had previously been one per week on average. (Hall 2008)

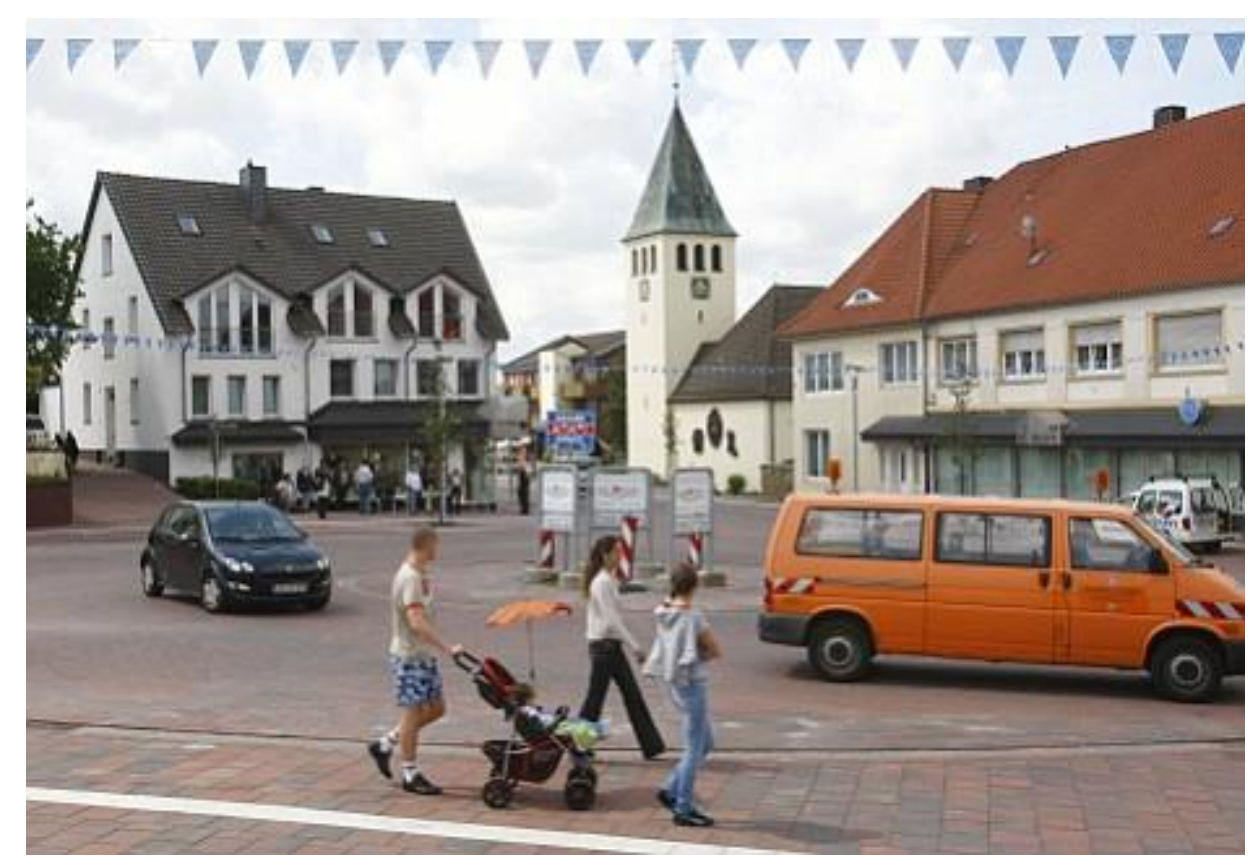

Figure 2: Shared Space in Bohmte, Germany (Hall 2008)

Another instance of shared space located within a town center exists in Poynton, England. (Figure 3) There, much like the other villages or small towns that considered implementing a shared space, the town was plagued by a congested arterial, 26,000 vehicles per day, running through the center of the town creating a hostile environment not welcoming to pedestrians and harmful to the local business environment. (Wilkinson 2014) After a complete renovation led by Hamilton-Baillie in 2011, the area became more welcoming for pedestrians, and won an award in 2013 for reducing congestion. (Wilkinson 2014) According to the 
Institution of Civil Engineers (ICE) the average speeds fell to $20 \mathrm{mph}$ without changing the posted speed limit, the travel times through the town center decreased, and only one minor accident occurred in the first three years compared to 4-7 in the previous three years. (Kirkup 2013) Economic benefits were also realized in this case, as in the years to follow the opening of the shared space businesses in the surrounding areas reported an increase in business activity by twice the previous amount. (Express 2013) Statistics reported by ICE stated that $80 \%$ of the retailers reported "increased footfall and turnover" and only one empty store location was present in February of 2013. (Kirkup 2013)
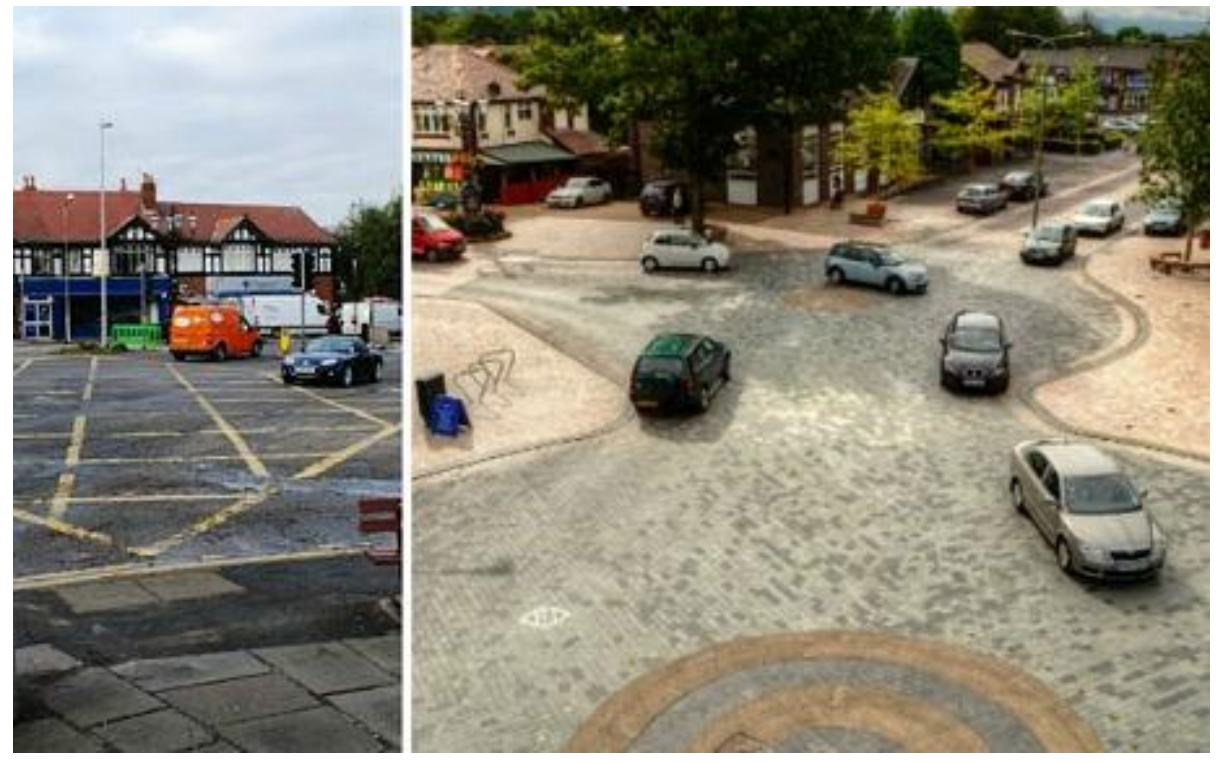

Figure 3: Poynton, England before and after shared space implementation (Wilkinson 2014)

\subsubsection{Other European Shared Spaces}

Small towns and villages weren't the only places found to be acceptable for the shared space design however. In urban downtown London, Exhibition Road which is home to many museums, cultural venues, as well as the Imperial College London, was transformed from a two way urban road to a shared space between 2008 and 2011. (Kaparias 2015) Similar to other shared space locations, Exhibition Road was crowded. In this case, not only was there a high 
vehicular volume (Kaparias 2015), but there was a significant amount of pedestrians and parked vehicles as well stemming from the 11 million annual visitors to the Exhibition Road area. (Access 2016) A recent study performed at the site found that the number of conflicts decreased both in number and in severity while comparing the before and after implementation data. (Kaparias 2013)

In Austria, another large city has had success with implementing shared space in a scheme to incorporate both pedestrians and bicyclists in with regular vehicle traffic. Graz, shown in Figure 4, is the second largest city in Austria and home to a large percentage of green space and pedestrian-oriented areas. In 2011, Graz chose to implement a new shared space design in Sonnenfelsplatz, within the downtown area and near a university campus. (Fischer 2011) The decision to transform the traditional urban intersection/pedestrian plaza in to a complete shared space allowed for an "improvement of spatial and social qualities", which strengthened the plaza's existence as a place or destination. (Recodrive 2011) Not only were local citizens more aware of the area as its popularity grew and likely to visit, the space also was able to exhibit safety aspects such as increased amount of social interaction and awareness amongst all of the users, resulting in no accidents for the first four months. (Fischer 2011) A study by Norman Garrick and Benjamin Wargos found that at this site, which exhibited a high level of freedom for the users, resulted in the lowest speeds measured by the vehicles in any shared space they studied. (Schmitt 2016) 


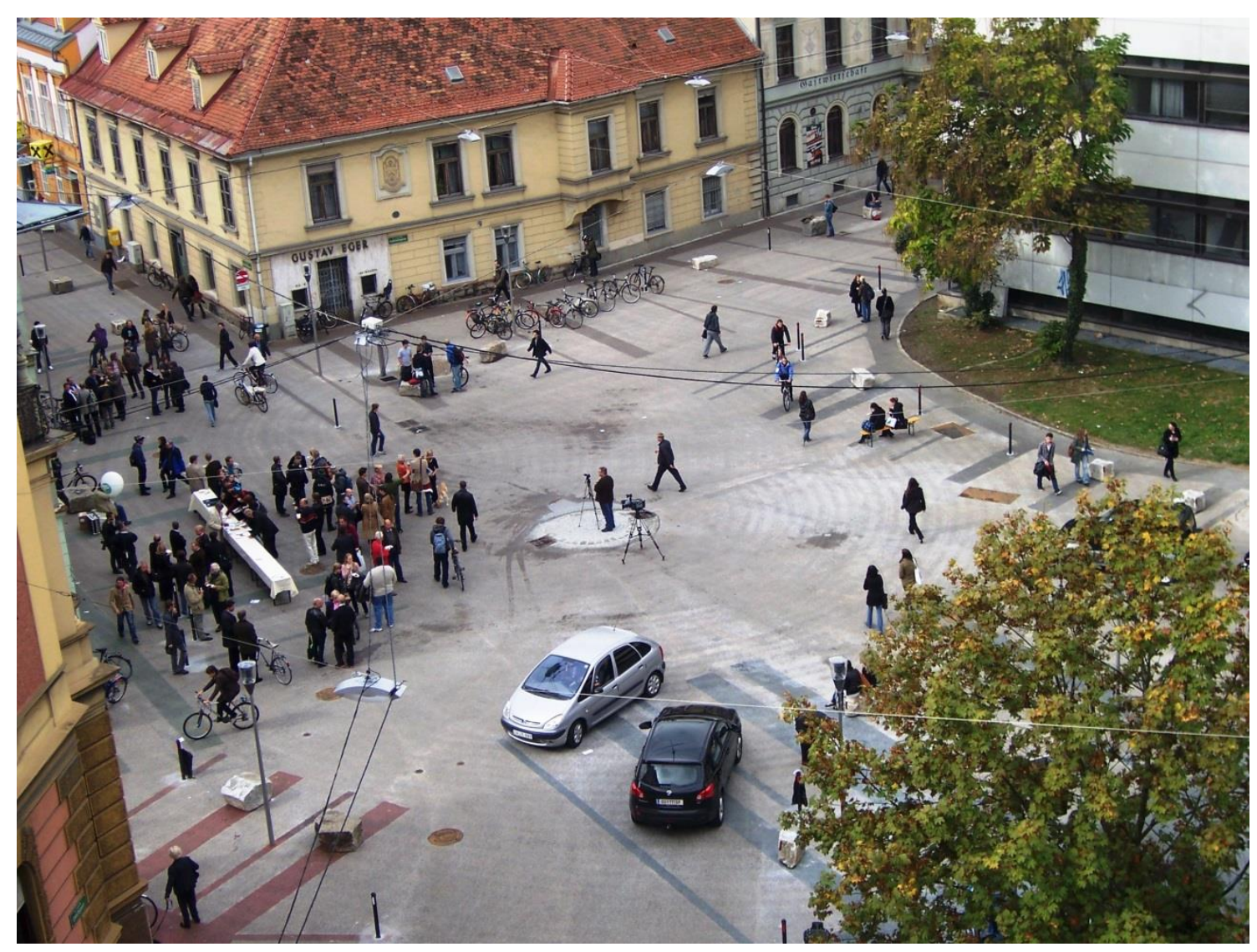

Figure 4: Sonnenfelsplatz - Graz, Austria shared space (Fischer 2011)

Finally, shared space has been successfully implemented in rural cases as well in the

Netherlands. The small town of Noordlaren has two main streets that cut through it, one of which passes right by the local primary school on the edge of the city limits. Unfortunately this location inevitably experienced inappropriately high vehicle speeds, and eventually an accident occurred. Rather than create more separation however, a shared space was created, which made the children at the school playground much more visible. This shared space, shown in Figure 5, included alternative pavements to capture the driver's attention and wide fields of view allowing the students and drivers to mutually be aware of each other. As a result, the speeds drastically reduced by approximately 6-7 $\mathrm{mph}$ at the location over the following five years. (Allianz 2012) 


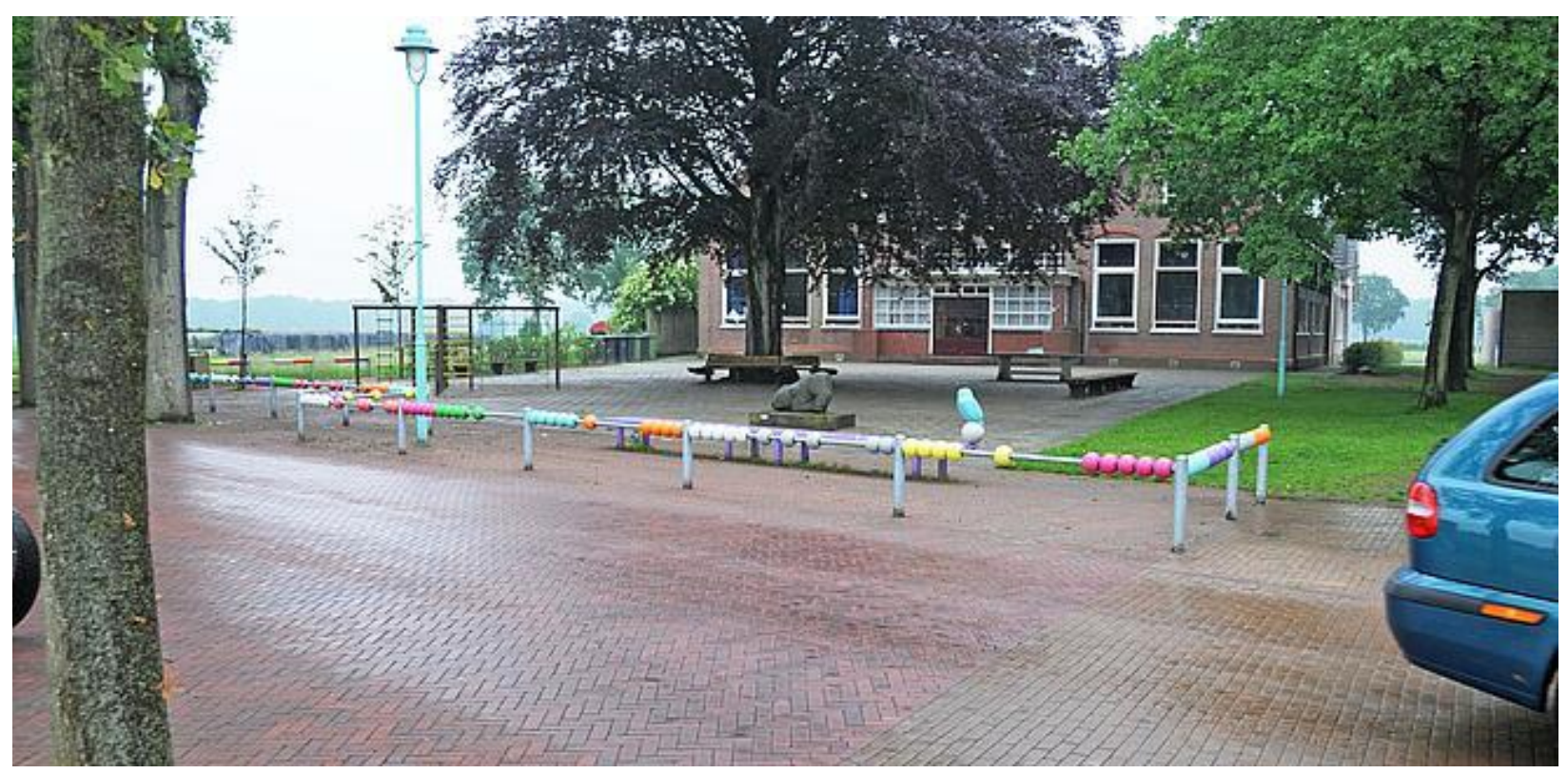

Figure 5: Noordlaren Shared Space in front of primary school (Allianz 2012)

As these and other examples have shown, shared space can be applicable in a variety of environments which share the same two basic characteristics of conflict between multi-modal users, and low vehicle speeds. As these results have shown, and will be discussed in further detail later, vehicle speeds are decreased, economic vitality is increased, and pedestrian safety is retained if not improved.

\subsubsection{Shared Space Outside of Europe}

Shared space has only recently begun to be implemented at locations in the United States, as well as other countries outside of Europe, and is certainly not as widely known or utilized as it is in Europe. Although these instances of shared space may not be as extensive, they too are also seeing successful implementations.

A recent thesis study completed in the US studied multiple components of shared spaces in the Pacific Northwest region. Bell Street Park in Seattle, WA, Davis Street in Portland, OR, and Santana Row Promenade in San Jose, CA were the three spaces being studied in this case. 
All three of these locations were located in urban areas, on secondary roads, with significant urban development surrounding them which provided destinations for transit, pedestrians, and bicyclists. Much like the shared spaces in Europe they all shared in common many of the design elements such as lack of curbs, non-traditional pavements, and absence of most traffic control devices. In this particular study, the transportation functionality, overall design, activity level, and user experience were surveyed. Overall, all three study locations were determined to function very well for drivers and pedestrians, with slightly lower functionality scores reported for transit and bicycle users. Identical results as were found in Europe were also mimicked in the US as well, as it was noted that vehicle speeds were kept effectively low and the "movement of automobiles was not stifled by the prominence of other travel modes on the street." As part of the user experience metric, safety of each area was also examined, of which all three earned the highest score since vehicle speeds were consistently low and pedestrian crossing was uninhibited. (Behrens 2014) We must note here that in this study, and many other studies, the use of shared spaces by individuals with visual or other impairments was often excluded. More research should be initiated to ensure these users are also accommodated in these facilities as they present unique challenges in navigating a shared space. 


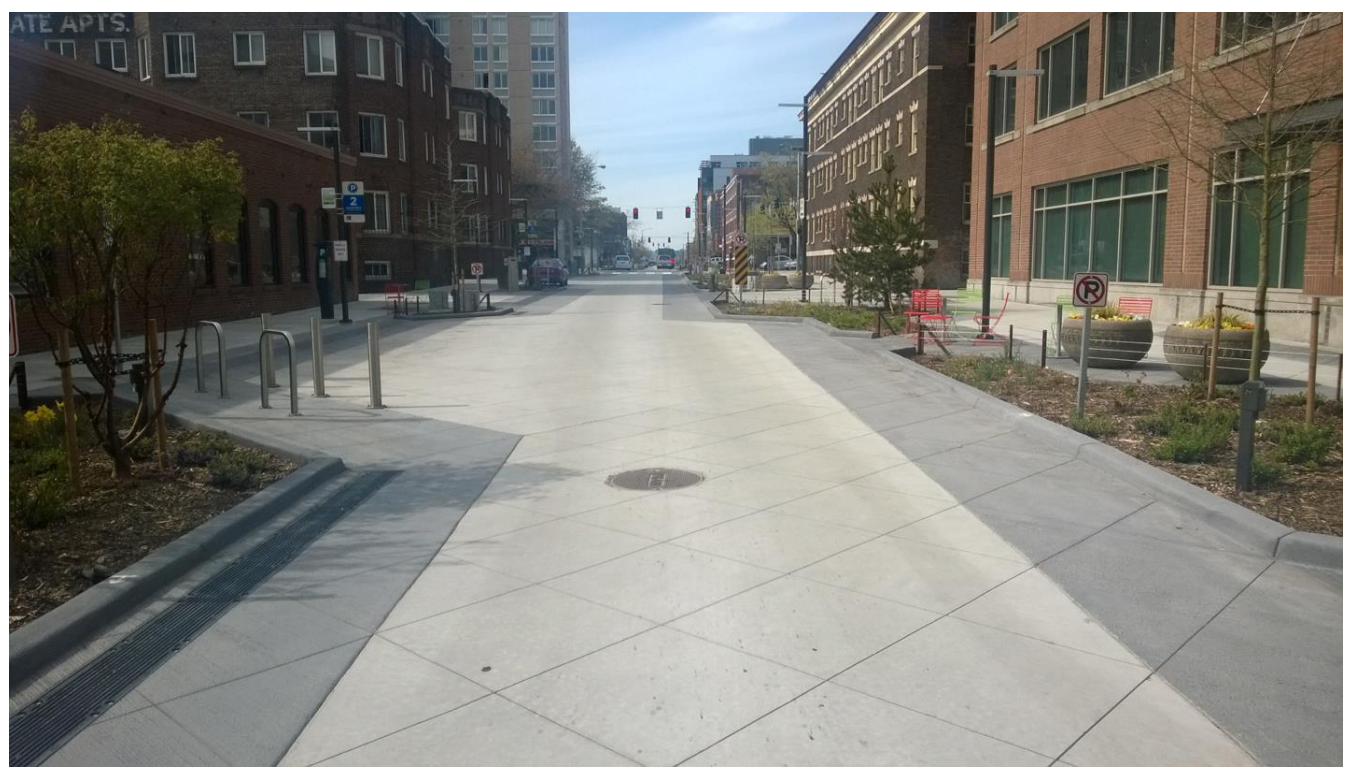

Figure 6: Shared Space on Bell Street in Seattle Washington (Behrens 2014)

Other instances of shared space in the US have shown up in the New England area and are being proposed in places like Pittsburgh, PA. In 2008, the city of Cambridge, MA converted two streets to shared spaces. These areas, mostly occupied by pedestrians and containing a posted speed limit of $10 \mathrm{mph}$ have been well received and are now vibrant with restaurants and pedestrian traffic increasing. These two designs were also able to live within certain US legal requirements all the while by using elements such as truncated dome detectable warning surfaces to comply with ADA regulations, a requirement that is not present in Europe. (Langdon 2010) Though these elements are one step in providing a safe environment for handicapped individuals, more research and design needs to be allocated towards ensuring these users can safely navigate and understand the unique right of way rules within a shared space. Meanwhile in Pittsburgh, Market Square, a historic plaza in the heart of downtown, was renovated to include some aspects of shared space. Much like the other instances of shared space, business and social activity has flourished. (Snyder 2014) The success of this project, and others, has now led to the proposal of an even more extensive shared space design on a major urban arterial intersection within 
downtown Pittsburgh. Liberty Avenue acts as an entrance off of the interstate in to downtown Pittsburgh, but also serves as a major intersection which serves a significant amount of pedestrians and bicyclists in addition to vehicles. In 2015 the shared space idea was proposed as a solution to the traffic chaos that was inherent at the intersection while creating a welcoming atmosphere for visitors that would also increase user safety. (Schmitz 2015)

Other countries are also considering renovating their existing streets or pedestrian plazas to incorporate shared space. In Australia for example, multiple instances of "shared zones and quasi shared spaces" can be observed. Barrack Street in Sydney, Australia, was noted in a recent thesis as one of the "most well-known examples" of shared space in Sydney. This location, although technically identified by signs and law as a shared zone, is rarely frequented by much vehicular traffic due to its design and layout which appears very pedestrian-oriented. Another shared space in Australia is located at Jack Mundey Place which again exhibits low vehicular volume due to the entrance design to the shared space that appears to discourage vehicles, although they certainly can enter the space. Finally, Chapel Road in Bankstown also serves as a suitable shared space example. The vehicles that did enter this space were noted to be cognizant of the different streetscape present and acted accordingly by driving slowly, even though there were no signs at the entrance to the space. (Gillies 2009)

Jordan has also been looking in to the use of shared spaces recently to combat a sort of identity crisis for roads like Al Medina Street in Amman. Roads like this were historically lined with consumer attractions like restaurants and shops, and still are today; however also now function as a main connecting artery between neighborhoods or districts. This increase in vehicle dominance has put more stress on the pedestrian and driver interactions. A recent study identified pedestrian crossing, vehicle parking, and traffic congestion as three of the main issues 
for Al Medina Street. In order to address these issues while maintaining access and mobility to the area, the study proposed a solution to create traffic relief routes or urban pockets thereby reducing the overall vehicular volume. The second part of this solution was proposed to possibly design sections of shared space along the street to redistribute the realm more towards an equal share, provide pedestrians with more social interactive space while enhancing safety, and give drivers the impression of acquiring more responsibility. (Tawil 2014)

All of these instances of shared space implementation or ongoing research and proposals for its eventual use show a progressive movement in the transportation industry to expand the metaphorical tool bag of alternative roadway design methods. This trend also highlights the shift in ideology as the planners and politicians are deciding to focus designs on all modes of users rather than the traditional vehicle centric methods. The successes of many of these individual shared space cases and the resulting benefits have provided traction for this concept moving forward. The benefits of safety, congestion relief, and economic revitalization, the main benefits provided with shared space have begun to be realized by the public and studied by professionals. The following sections will expand on the previously mentioned statistics and studies to provide a more technical review basis of these benefits.

\subsection{Shared Space Safety Research}

In all of these discussed shared space implementations, increased pedestrian safety has been at least one component to why shared space was chosen as a suitable alternative in addition to other benefits. Much of the reason shared space provides a safer pedestrian environment is the way in which it changes the interaction between pedestrian and vehicle users of the location. Social interactions governed by the user's own risk assessment become the traffic controlling 
device rather than a light or sign. The physical aspects of a shared space combined with this cognitive change are able to reduce vehicle speed, conflicts and injuries.

\subsubsection{Risk Assessment principles}

By taking away the traditional forms of traffic control, including signs, lights, and other engineered implements which gave drivers and pedestrians informational cues, a higher and much more apparent level of uncertainty is created for the users. As Charles Landry says, this increase in uncertainty in any situation and a perceived notion of powerlessness over an event raises our internal risk-consciousness. (Cabe 2005) As was discussed previously, the main pillars of shared space designs create an unlimited array of pedestrian origin-destination patterns, allow vehicle drivers to make their own decisions through social means, and provide most users the same claim to the road. Again, we must note that cognitively and visually impaired individuals may experience difficulty in navigating a shared space. All of these create a very uncertain scenario for a user entering the area, and by not having defined rules or priority, leave the users perhaps feeling powerless as they must rely on communication and social norms to navigate a street rather than clearly defined laws and regulations. Although this sounds like a daunting task, it captures users' attention and triggers their risk awareness. As one's risk-consciousness, or awareness, rises, they begin to view their surroundings through the risk assessment process.

This process of assessing one's risk and the appropriate action takes much observation, weighing of options, and decision making, which all relate to time needed to perform these mental tasks. One risk assessment approach, known as STEM, involves finding the Sources of the risk, it's Target, what the adverse Effect would be, and by what Mechanism does the effect increase in probability. Another four component approach is summarized as looking for the 
hazard, assessing the consequences, followed by the respective likelihoods, and finally characterizing the risk. (Taylor 2012) In terms of driving or walking through a shared space, these methods relate to the user gathering information constantly as his or her observations change with the movement of oncoming vehicles and crossing pedestrians. As an effect, drivers begin to slow down and proceed with more caution creating a safer environment for all users since this process takes a considerable amount of time to successfully complete.

This however, is in stark contrast to the current practices within roadway design. As Hamilton-Baillie points out, risk is currently seen as a quality that must be minimized to successfully pursue safety. (Hamilton-Baillie 2008) This has been accomplished by creating segregated facilities with curbs, signs, bridges, and additional infrastructure taking many of the decisions out of the equation for the user. By creating a seemingly risk-free environment, users may be incentivized to perform additionally risky activities since the original risk is not realized. (Hamilton-Baillie 2008)

This principle is known as the Peltzman effect, and was hypothesized by Sam Peltzman in 1975. The Peltzman effect "raised the question about the efficiency of ... mandatory safety regulations" that were put in place by the US federal government in 1966 via the "National Traffic and Motor Vehicle Safety Act”. (Diosdado-De-La-Pena 2008) Through a study conducted by Peltzman, he was able to conclude that although the probability of driver death did decrease, the fatality risk for pedestrian increased. This was all due to what he referred to as drivers meeting their risk equilibrium. As vehicles became safer, drivers exhibited a higher propensity to drive with a riskier behavior. (Diosdado-De-La-Pena 2008)

Other more recent studies have shown similar trends with the increase in SUV use. This particular study used FARS data to compare the amount of passenger vehicle-passenger vehicle, 
passenger vehicle and SUV, and SUV-SUV crashes over a 10 year period. Due to the higher perceived safety from the point of view of a SUV driver (higher mass, stiffer steering, higher seating position), riskier behaviors were performed resulting in an increasing amount of SUVpassenger vehicle crashes, while the SUV-SUV crashes remained constant and passenger vehicle-passenger vehicle crashes decreased. (Diosdado-De-La-Pena 2008) Finally, a recent study completed at Texas A\&M showed that enacting mandatory seat belt laws also exhibited the Peltzman effect as drivers behaved in a riskier manner. However, it was noted in this study that the effects were found to "fade away over time" as wearing seat belts become a normal behavior. (Texas A\&M 2015) Shared space, by creating what would be perceived by drivers to be a riskier environment, can make use of the Peltzman effect in the opposite manner. As the higher risk is perceived, drivers will shift their behavior to be less risk taking, and therefore meet their equilibrium. Since shared space is a sharp contrast to the rest of the built environment, it will most likely not be subject to the effect fading away as Texas A\&M found with seatbelts.

John Adams also stresses that the clutter created by commonly segregating traffic flows has allowed for vehicles to move faster in congested areas and has little proven safety benefits, rather creates information overload. (Cabe 2005) Shared space is the "antithesis", as Adams says, to this traditional notion that users are "obedient automatons". Rather shared space designers and proponents have shown that humans are "alert to signs of safety and danger and modify their behavior accordingly", and in the end shared space can create a safer location by allowing the users to "look out for, and respect" the other users. (Cabe 2005) This need to "look out for" other users and the general ambiguity surrounding shared space presents a challenge for impaired individuals and needs to be addressed in future designs. 


\subsubsection{Reducing Vehicle Speeds}

As discussed earlier, it has been proven through crash statistics that with lower vehicle speeds, there are fewer occurrences and lower severity in pedestrian collisions. In addition to this, studies have shown that $40 \%$ of all crashes are caused by excessive speed factors, and that pedestrian deaths account for approximately one sixth of all road fatalities. (Gillies 2009) If new infrastructure that is designed to service both pedestrians and vehicles can be proven to reduce the average speeds of vehicles, it can certainly be a safer environment. Shared space has been proven to both reduce the vehicle speeds, and in turn reduce the number of pedestrian-vehicle conflicts.

A comprehensive study of many locations in the UK observed the operational characteristics of each intersection or link which was deemed to contain elements of shared space. Based on their physical structure and the demand they serviced, they represented a wide range of shared space type of facilities within the UK. The elements which were studied at these locations included the pedestrian traffic patterns, propensity of drivers or pedestrians to give way, types of conflict, and vehicle speed.

In terms of keeping vehicle speeds low, this study is a prime example of the suitability of shared space to increase pedestrian safety. As seen in Figure 7 below, across the majority of the ten studied locations with varying degrees of shared space implementation, the observed average speed of vehicles was no more than 15 miles per hour. This alone is a very promising fact; however it helps establish the speed reduction claim by noting that most of the facilities had posted speed limits above 15 miles per hour. Even the locations which did not have average observed speeds below 15 miles per hour, still showed speeds much lower than the posted speed limits. (Shore 2010) This shows the true ability of shared space to entice drivers to behave 
according to the surroundings rather than the mandated traffic control. Also, an average speed of less than 15 miles per hour coincides with the studies which have found 15 mile per hour to be the safety threshold for pedestrian vehicle interactions.

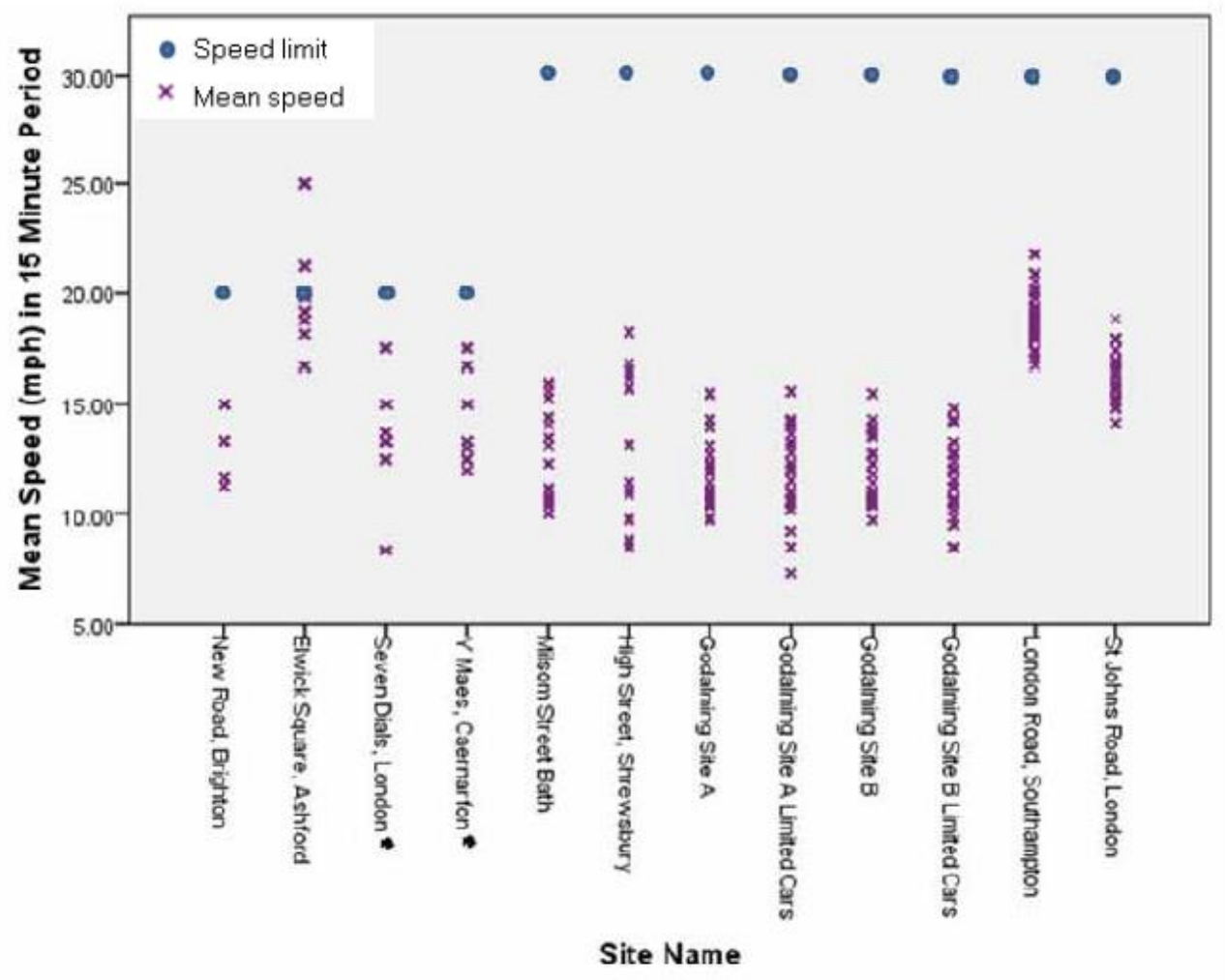

Figure 7: Mean Speed at each 15 minute period, by Site (Shore 2010)

In a separate study of the Monderman designed intersections in Oosterwolde and Makkinga, Netherlands, it was found that even with a slight drop in vehicle volumes, there was on average a 20 kilometer per hour decrease in vehicle speeds. Another study of the Laweiplein intersection in Drachten, Netherlands showed that the site exhibited an $82 \%$ decrease in accidents in the first year by reducing the frequency of 11 accidents per year to 2 accidents per year. (Gillies 2009) Through these studies we can see that the theoretical increase in safety by reducing speeds has been exhibited as a capability of past shared spaces. 


\subsubsection{Reducing Conflicts between Pedestrians and Vehicles}

The comprehensive shared space study of ten locations across the UK was also able to capture the change in pedestrian and vehicle behaviors within a shared space, especially their interactions. Beyond observing the speeds and volumes of vehicles and pedestrians, this study also examined the origin-destination (O-D) travel patterns, propensity of users to give way, and the severity of encounters between users. An additional set of studies of Exhibition Road in London also examined the change in vehicle and pedestrian conflicts. These results again point to the success of shared space in multiple locations.

In the comprehensive study, conflicts between vehicles and pedestrians were tracked and rated on a four tier scale based on whether one or both participants in the encountered moved, and whether that move was sudden or not. Across all ranges of vehicle flows through the shared spaces on average the encounters only required one participant to move $88 \%$ of the time, which corresponded to the lowest encounter severity tier. The shared spaces which exhibited medium traffic volumes had the lowest amount of level 1 encounters at $77 \%$, while the low vehicle volume shared spaces had the highest amount of level 1 encounters at 95\%. (Shore 2010) This conceptually makes sense as the volume of vehicles increases there will be a higher chance of encounters between users. It is an excellent sign to see that the vast majority of all encounters are rated at the lowest severity level, providing the least amount of risk for users. The remaining encounters were split between the level 2 and 3 tiers almost evenly, and only $1 \%$ of encounters reached the level 4 severity with both participants suddenly moving.

At another specific location in the UK, Exhibition Road in London, the severity level of conflicts between users was tracked and compared between data collected prior to the completion of the shared space design and following its opening. Both iterations of the data 
collected by Kaparias et al., and then by Dong, once again reiterated the proven safety benefits of shared spaces. The method used in these studies captures four factors of the conflict between a pedestrian and vehicle. Those four factors: time to collision (A), severity of evasive action (B), complexity of evasive action (C), and distance to collision (D), are given a rating between 1 and 3 for factors $\mathrm{A}, \mathrm{C}$, and $\mathrm{D}$, and 1 and 4 for factor $\mathrm{B}$, with the higher rating corresponding to a more severe/complex maneuver or shorter time and distance to collision. (Dong 2012) Each factor then results in a separate rating for each factor. Through a grading chart devised by Kaparias at el., the four separate ratings are then combined in to a single conflict grade between 1 and 4. Again 4 corresponded to the highest severity conflict.

The results in the Kaparias study showed that the main conflict recorded were the grade 1, slight conflicts, before and after the shared space implementation. In both cases the remaining conflicts decreased with increasing severity. Overall, the amount of conflicts decreased slightly over the entire area. It was noted by Kaparias et al. however that a more meaningful comparison would be had by normalizing the occurrence rates of the conflict with the volume of pedestrians crossing the street. This parameter changed dramatically with the implementation of shared space as pedestrians now had increased access within the study location. When this comparison was performed, a much more drastic decrease in overall conflicts was noted, as well as for each of the individual severity levels as well. (Kaparias 2013)

Dong's report found similar results, but correlated the data to total risk and expected risk values as well. The total risk value at Exhibition Road decreased by $20 \%$ between the two time periods while the expected risk value only increased by an insignificant 2\%. (Dong 2012) Again, Dong normalized these values with the pedestrian crossing volume, and in addition the vehicle flow. Just like in the Kaparias study, these normalized values captured the true change in total 
and expected risk. The data collected showed an approximately $20 \%$ decrease in vehicle volume, while a $50 \%$ pedestrian crossing volume increase occurred. On a pedestrian crossing normalized basis, the total risk dropped by more than $50 \%$, and the expected risk almost $50 \%$ as well. (Dong 2012) Because the vehicle volumes actually decreased, the total risk value did not decrease as dramatically in this comparison.

By observing the same amount of conflicts or fewer, with an increased level of pedestrian activity, these findings bode very well for shared space as an alternative to provide a safer infrastructure for pedestrians. All of these findings discussed were conducted in Europe, where shared space has been implemented in many countries. However, many of the studies were conducted shortly after the spaces were opened to the public or within a few years. Future studies tracking these changes over an extended period of time will help solidify the safety benefits of shared space by proving the results are not simply a short-term effect.

\subsection{Economic, Social, and Traffic Benefits}

In the first section of this literature review as past implementations of shared space were discussed across Europe and countries outside of the region, safety benefits were noted most frequently as being the motivational factor, or the largest perceived benefit of the completed projects. Many of the sites also noted benefits in other areas as well including economic revitalization, social perception, and traffic flow benefits. By adding these benefits to the already verified safety benefits

\subsubsection{Economic Revitalization}

Unfortunately at the current time, not many scientific studies or peer reviewed articles exist to support or refute these claims. However, through anecdotal evidence and media reports 
we can capture positive feedback from users and shop owners in the vicinity. For example, in Poynton UK, a brief survey of local shop owners returned results that $80 \%$ of them claimed to be experiencing increased footfall and turnover of customers. (Kirkup 2013) Conceptually these results make sense and are in line with previous discussions. By making the space more safe for pedestrians, and allowing them equal access with vehicles to the area, the designers are encouraging the use of the infrastructure by this demographic which has for long been pushed aside and separated with traditional traffic control methods as described by Hamilton-Baillie. (Hamilton-Baillie 2008)

Studies like that completed by Dong, have shown these assumptions to be true. In that specific data, over the course of a few years, the pedestrian volumes on Exhibition Road increased by a dramatic 50\%. (Dong 2012) These increased levels of pedestrians can then be associated with increased sales and economic traffic as well in a specific area. A recent study completed in Toronto to assess the future of on-street parking, cycling, and pedestrian facilities reinforce this notion with its findings. One portion of this study looked at the customer base for stores in the central business district of multiple North American cities like Toronto, Vancouver, New York, and Portland. The data resoundingly showed that the vast majority of customers who visited shops in the selected locales arrived on foot or bicycle. In New York alone, nearly $95 \%$ of customers arrived by either of these means, and on the lowest end of the spectrum, Portland stores received customers via these means still more than $65 \%$ of the time. (Arancibia 2013) Another report in Vermont quantified the value of a location being considered walkable. Their data showed that locations classified as walkable have an increased value of $\$ 6,500$ over properties in car dependent areas. (Resource 2012) Shared space certainly can excel in a central business district environment with these characteristics, and it does not stretch the imagination to 
see how these attributes can also serve campuses, small towns, and other areas with high pedestrian demand suitably as well.

\subsubsection{Public Perception}

Mainly through brief survey data, as referred to previously through the individual shared space descriptions, can we see the positive perception the public has of shared spaces implemented in their towns and cities. One example is in Norrköping, Sweden, where a shared space was implemented in 2004. Initial surveys show an increasing satisfaction and confidence rating given by drivers, cyclists, and pedestrians. (Gillies 2009) A specific study completed by Kaparias et al. sought to capture the specific perceptions of pedestrians and vehicles towards shared space. This study created a logit model based on binary responses to a survey completed by respondents online.

Questions in the survey gathered information on internal and external factors like age, gender, and disability, as well as safe zones, vehicle traffic, and lighting level respectively. Respondents were given multiple scenarios based on the previous binary factors and were asked whether or not they would be comfortable driving or walking in this environment. The results showed that just over half, $51 \%$, of the scenarios were deemed comfortable for pedestrians. Meanwhile, $54 \%$ of the respondents placed in the scenarios as drivers responded that they would be comfortable and willing to share the space with pedestrians. (Kaparias 2012) One interesting demographic that should be noted in this study though, and most certainly would have had an impact on the responses, is that $55 \%$ of the pedestrian survey respondents, and $29 \%$ of the driver survey respondents had not heard of shared space designs prior to their participation in the survey. Additional, only $44 \%$ of the driver survey respondents had experience driving through a 
shared space. (Kaparias 2012) Therefore, their only knowledge of the type of environment was a brief introduction and description at the beginning of the survey to inform the subject on what shared space is.

A regression model was then fit to the data to determine what aspects or qualities of shared spaces provided the users with more comfort for using the area. Some of the findings showed that the presence of safe zones greatly increased the comfort of pedestrians. For drivers, an increased pedestrian volume, especially with elderly and small children, decreased their comfort and willingness to share. Meanwhile, this increase in pedestrian volume makes the pedestrians themselves much more comfortable with the surrounding. Kaparias notes that these findings could shape future shared space policies by ensuring safe zones are required, and encouraging more pedestrians to use the area to "ensure the enhanced alertness of drivers" since they would be less willing to share. (Kaparias 2012)

This study captures the ability of shared space to create equilibrium between the users and promote the location's use by multiple modes of transportation. Although all signs are positive from the current research, future studies should look at specific cases of shared space, as well as before and after studies of public acceptance with the shared space.

\subsubsection{Traffic Congestion}

Perhaps the most untapped potential benefit yet to be extensively measured is shared space's ability to reduce traffic congestion, and create smoother flows of traffic. As can be seen in the previous discussion, the main focus is safety. Claims have been made though by users in the past that shared space has reduced their delay and congestion experienced at an intersection. The MVA comprehensive study of shared spaces in the UK captured a few characteristics and 
behavioral patterns of users which lend themselves well to allowing for a more efficient use of the intersection.

As is easily noted in a shared space scheme, there are no identifiable cross-walks or specific locations for pedestrians to cross. With this absence of markings in addition to the lack of curbs and other traffic control devices, pedestrians now have the opportunity to cross anywhere within the space. The MVA study pinpointed the origin-destination (O-D) patterns of pedestrians crossing through their selected shared spaces. These O-D patterns were classified based on whether or not they were on the user's desire line/shortest path. In the locations that were classified as true shared spaces, $80-100 \%$ of the pedestrians crossed on their desire line. In comparison, the sites that were more conventional exhibited only 50-60\% of desire line usage. (Shore 2010) This shift in pedestrian movement pattern results in a larger spread of pedestrian routes and volume across the entire area, rather than having all pedestrian crossings centralized at a select few locations. By spreading out the pedestrians, larger gaps between individual pedestrian crossers are prevalent. These larger gaps provide a higher probability that a vehicle could progress through the intersection between two pedestrians.

The MVA study also tracked the propensity of drivers and pedestrians to yield to one another, shown in Figure 8 below. In overall cases, the number of encounters in which the pedestrian yielded to the vehicle was approximately $70 \%$, and conversely the drivers yielded to pedestrians about $30 \%$ of the time. Interestingly, interactions between cyclists and vehicles were split 50/50 for which user performed the yielding maneuver. Since this data was collected over ten separate sites, the site specific yielding patterns varied greatly. For example, the percent of vehicles giving way to pedestrians ranged from $55 \%$ at Seven Dials in London, to $5 \%$ at London Road in Southampton. (Shore 2010) 
MVA then further analyzed the data to determine patterns in drivers yielding based on average speed. It was determined that as the average speed decreased, the propensity of drivers to yield to pedestrians increased. Likewise, as the vehicle speeds exceeded 16 miles per hour, the amount of drivers yielding to pedestrians began to decrease.

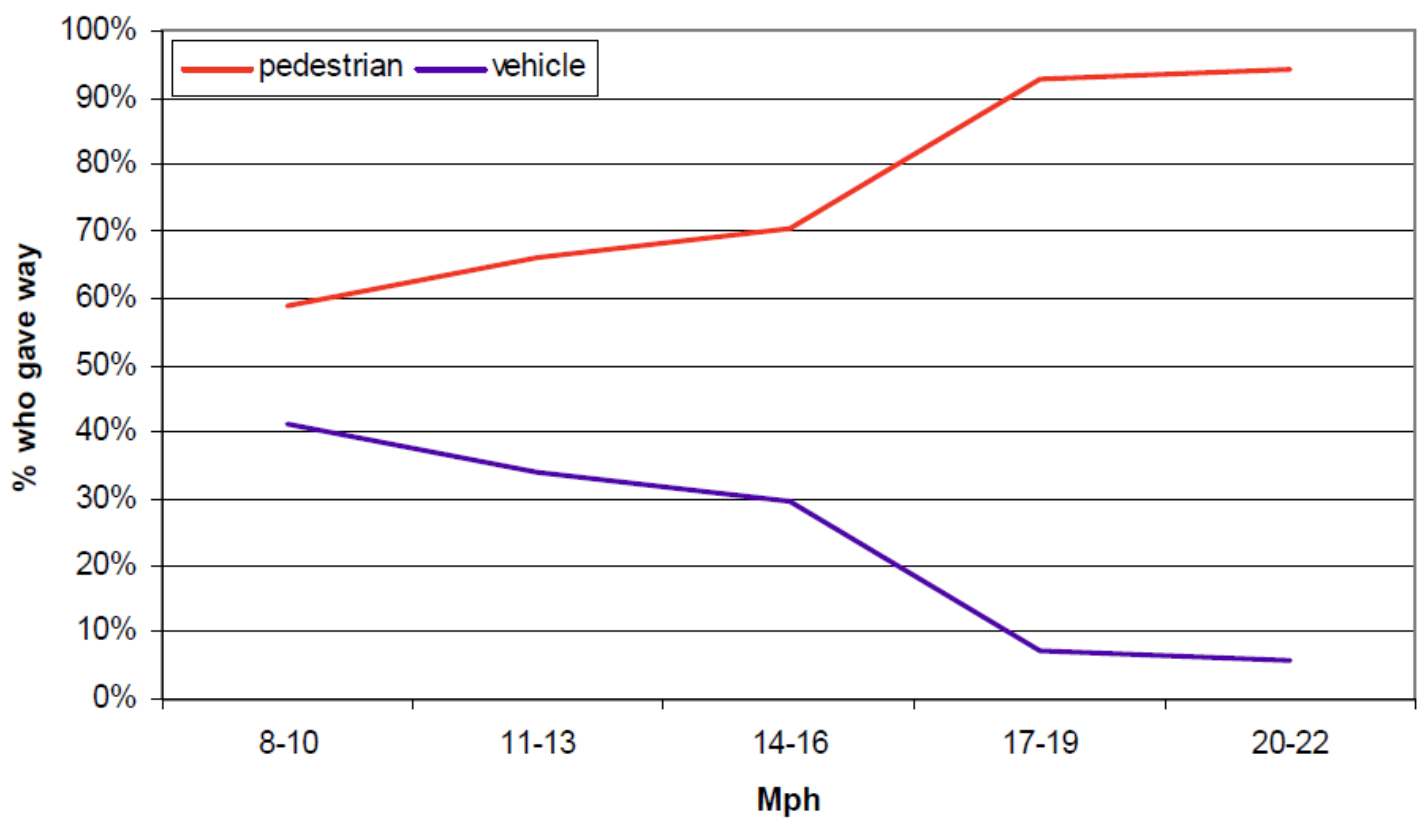

Figure 8: Proportion of users giving way based on average speed (Shore 2010)

A second correlation was made between the vehicular volume and proportion of users giving way, shown in Figure 9. The largest proportion of drivers yielding to pedestrians occurred during the 15 minute periods with vehicle volumes of 26-50 vehicles, at which time drivers yielded $40 \%$ of the time to pedestrians. This $40 \% / 60 \%$ split was the closest the pedestrian and vehicle proportions became in either correlation. Once the vehicular volume increased beyond 50 vehicles per one 15 minute time period, the proportion of drivers yielding quickly decreased to the point of $5 \%$ when the vehicle volumes exceeded 150 . 


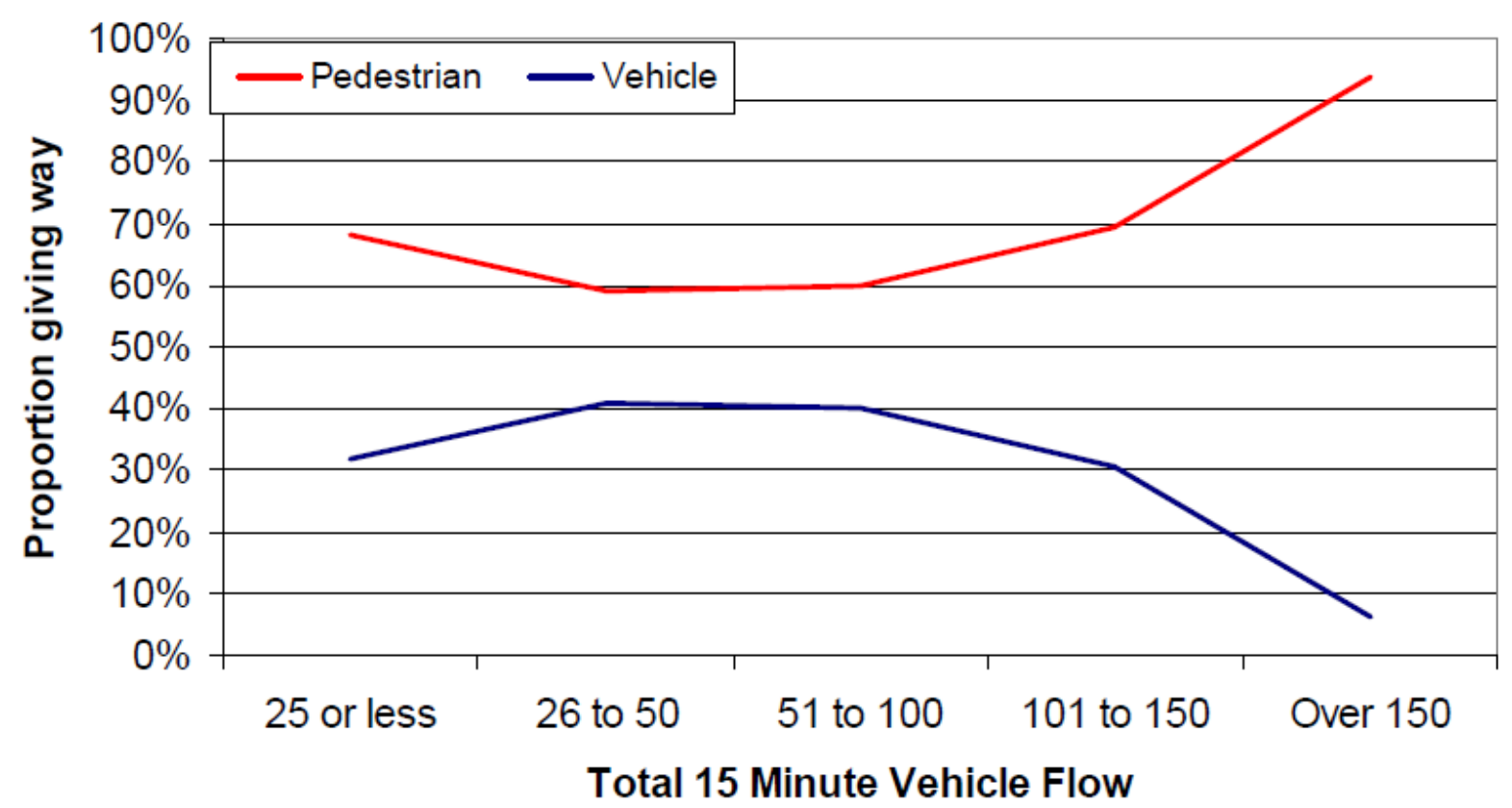

Figure 9: Proportion of users giving way based on total 15 minute vehicle flow (Shore 2010)

The previous proportion of yielding however assumed the pedestrian volumes to be even during each 15 minute time period. Therefore, another correlation was completed based on the pedestrian flow. These results, shown in Figure 10, showed that the proportion of yielding reached an equilibrium of 50\%/50\% when there was between 20 and 34 pedestrians in the shared space area during the encounter. At pedestrian flows above this level, the vehicles yielded to the pedestrians more often, whereas at pedestrian flows below 20 the pedestrians began to yield more frequently. In all cases, the proportion of yielding did not exceed $75 \%$ for either group. 


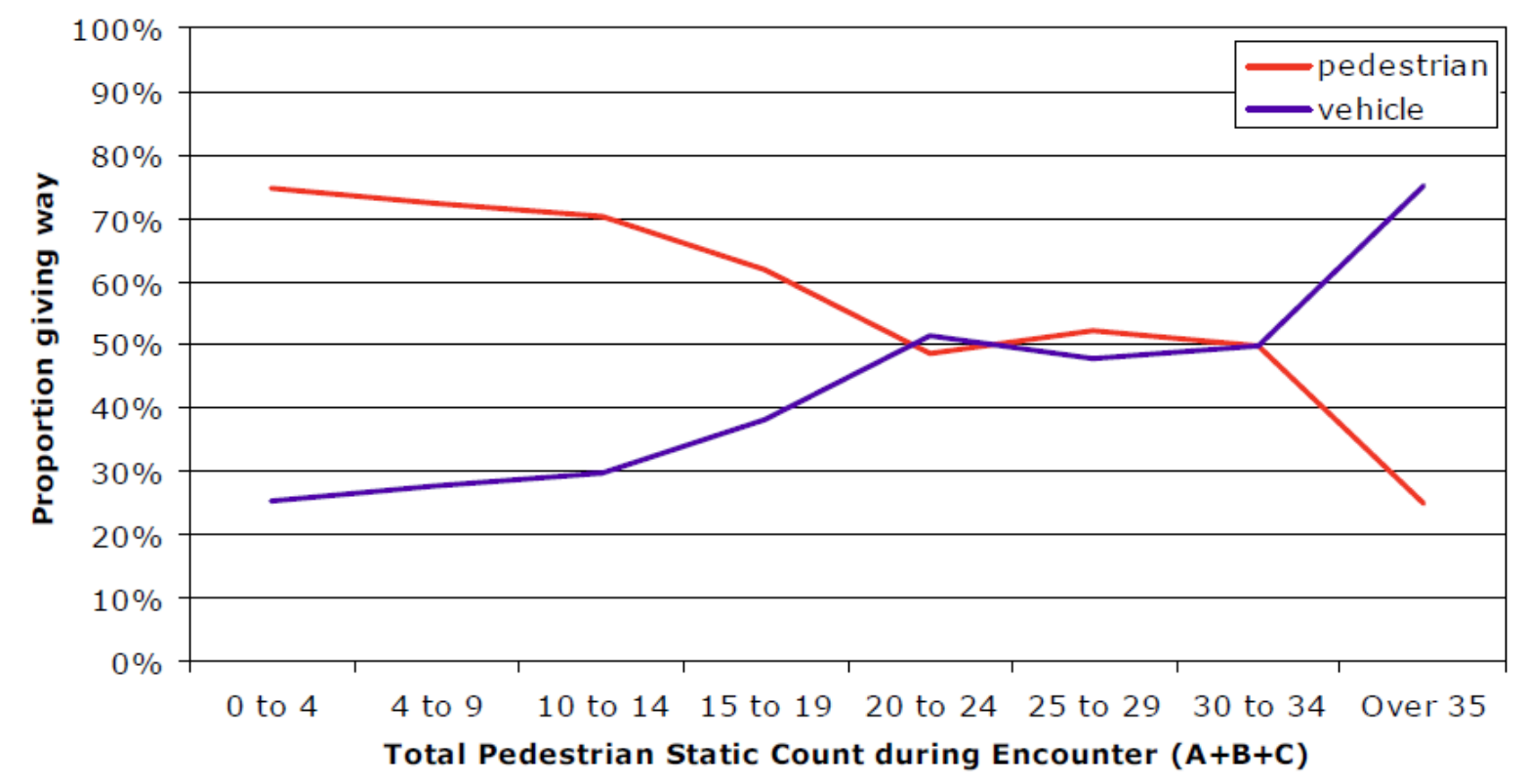

Figure 10: Proportion of users giving way based on total pedestrian count (Shore 2010)

Although this data doesn't specifically track the delay savings for vehicles or pedestrians, we can conceptually recognize that with a lower proportion of yielding, that group of users will incur less delay on their travel through the shared space. It also serves again to show the dynamics of shared space behavior and the balance between pedestrian and driver activity. The lack of before and after delay studies surrounding shared space projects should be remedied in the future with research on the effects shared space has on queues, travel times, delay, etc. for drivers. This method of researching a completed project, however, requires significant capital and time investment. Therefore it presents the need for the research study performed in this thesis, to devise a way in which shared space concepts can be tested in various locations with different vehicle and pedestrian volume and speed characteristics to determine its future benefits and implications on the specified area. Current modeling methods for shared space are briefly described below. 


\subsection{Modeling Shared Space}

As shared space alternatives continue to be viewed as alternatives to intersections, boulevards, and to solve traffic problems, there is an increasing need for them to be modeled prior to construction. These models can help predict the efficiency benefits of a shared space infrastructure in a particular situation. As has been seen throughout this literature review, shared spaces are very dynamic traffic environments whose efficiency depends on the relative pedestrian and vehicle volumes, physical infrastructure, and O-D patterns for all users. Microscopic simulation methods are currently used as an industry standard to model intersections and small networks, and can act as a suitable starting point for shared space models. (Schonauer 2012) Although software packages exist for planners to conduct said simulations with traditional infrastructure and social behavior, only experimental simulation methods have been devised at this point. Currently, these are mainly based off of the social force model approach which allows individual vehicles and pedestrians to behave according to forces imposed on them by other users to avoid collision and maintain their required path, like a car following model or rule based approaches. (Schonauer 2012)

\subsubsection{Social Force Approach}

Studies completed by Schonauer et al., Pascucci et al., and Rudloff et al., all devised similar modeling methods of shared space based off of the social force model. Although their individual techniques were slightly different, the premise, concepts, and results of their tests are very similar. These results were all positive as well, proving that it is possible to closely reflect via a model the real-world behavior of pedestrians and vehicles. 
In the model created by Schonauer et al., each mode of user was modeled separately via microscopic simulation, in a model comprised of three main layers. These layers consisted of a infrastructure, operational, and tactical model. The infrastructure model takes the users intended origin and destination, then creates a path to their intended goal based off of the overlaid infrastructure. First, the physical environment was split in to straight and intersection portions of the shared space. The elements of environment were then assigned force vectors to keep individual users on track and avoiding obstacles. This method results in a lateral distribution of possible paths, each with their own value for attractiveness to the user. Without being acted upon by other users, this most attractive path based solely on the infrastructure will be the path the user takes. (Schonauer 2012) This differs from traditional microscopic models which have much fewer degrees of freedom for their users. In that case, routes are statically defined by the planner and vehicles or pedestrians will follow lanes and paths to reach their destination, in contrast to the open areas present in shared spaces.

The second layer in the Schonuer et al. model was an operational model based off of the multi-agent social force model, paired with vehicle dynamic models to prevent cars and bicycles from making unnatural movements. This portion of the model includes basic social interactions for users to make small directional changes. Users, also known as agents, as well as infrastructure elements emit individual force fields to sustain appropriate distances between each other. The acceleration force of the vehicle to reach its goal, summed with the forces of the other agents and infrastructure create the social force.

Finally, the tactical model level is required to ensure a better fit of the model to the empirical data. In shared spaces, vehicles and pedestrian must interact socially and react from distance in excess of what can be accomplished through the social force model. For this purpose, 
the tactical force is added to the existing forces, guiding field and social force, to create the final individual agent force. The tactical model is run through a process of conflict detection, handling, and game theory. In conflict detection, other agents within a predetermined radius are detected by the agent. Then, through conflict handling the game theory commences by creating a simple game between two players based off of a technique by Li and Chen, with the possibility of five choices. (Schonauer 2012) These choices are for the agent to remain on path, accelerate, decelerate, or swerve left or right, each with its own payoff value. This game is designed to be rational in a Stackelberg nonsymmetrical hierarchical fashion, with a leader and follower agent. In simple terms, the leader agent makes a move to which the follower agent will then react. The follower in the game theory reacts to the leader by estimating their individual payoff of the five possible choices. This is conducted through an estimation utility comprised of collision avoidance, social utility, saved detour, energy loss, and normative utility, from which the highest weighted sum of all utilities will be selected as the preferred decision. . (Schonauer 2012)

In tests of this model, 60 pedestrian trajectories crossing a road were selected from video data, including 15 potential conflicts. The model was run through this data set with the underlying assumption that the pedestrian in this case would always be the leader. By tracking the behavior of the follower agent, the oncoming vehicle, the weights of each utility function were able to be estimated. The results showed that this model can accurately predict the paths of vehicles in a shared space environment, however it was noted that more research would be needed to predict the paths of bicycles and pedestrians since the selected location had a relatively high amount of vehicular traffic.

The second model was based off the initial Schonauer at al. model with similar operational, tactical, and infrastructure models. It was noted in this model that the social force 
model utilized as the base was taken from PTV Vissim, an industry wide utilized microsimulation software. This model however was calibrated using data from two shared space sites in Austria in Gleinstätten and Graz. From video data collected, clips with conflicts present were identified summing 61 samples. (Rudloff 2013) The force fields emitted by agents were calibrated using the data collected by applying a beta distribution to the lateral position and forming the guiding line. Next, the tactical game was calibrated using the log likelihood of estimated parameters. Rather than assume the pedestrian was always the leader as in the last study, this method used an explorative manner to determine who the leader was. Some of the defining criteria included which agent was closer to the conflict point, who was faster, and who had the right of way according to traffic regulations. Once the model was calibrated, it was then compared to real-world data from Sonnenfelsplatz in Graz.

In Graz, once the shared space design was implemented pedestrian paths became shorter and closer to the square's center, and also showed a higher variation for where the paths crossed the driving surface. (Rudloff 2013) The paths predicted by the model followed this similar pattern, however tended to exhibit less variation or be more "channelized" as Rudloff explains. (Rudloff 2013) It was also noted that the simulated paths exhibited more turns or adjustments to the paths rather than a natural curvature as was observed in the actual pedestrian behavior. Other metrics were compared between the simulated and observed data as well including speed of pedestrians, vehicles, and bicyclists. The results showed that the data was similar between the observed and simulated trials. It was noted however that the pedestrian speed distribution was relatively narrow and vehicle speeds exhibited a comparatively larger spread since the pedestrians were more likely to avoid conflict by altering their path direction rather than speed, and vehicles were mostly restricted to alter their speeds to avoid conflicts. 
The Rudloff study made a few conclusions including the preference of leaders, important utility factors, and safety predictions. The study found that by tracking the relative speed and distances between users, a hot spot map could be created to identify areas of potential high risk for conflicts. In terms of the model choosing a leader, it was found that a slight preference was made to the subject who was closer in terms of time to reaching the conflict point. Finally, it was determined that the socially acceptable parameter portion of the utility played an important role in dictating the user's decision. (Rudloff 2013)The findings from this study can now be used to improve future models and begin to add data collection and prediction levels to the model so that efficiency metrics such as delay and level of service can be determined.

Finally, a microsimulation model by Pascucci et al. was created, again based off of social force model principles. This model was created to fill a gap in shared space simulation capabilities by providing planners a way to estimate the level of service, delay, and road capacity of proposed shared spaces. To begin, the behavior of users at an intersection in Braunschweig, Germany, was tracked to determine the change in user trajectories. The data was analyzed to identify the functional area in which the user was present, their free flow trajectory, and the collision avoidance mechanisms they used. Some patterns were identified, for example pedestrians choose the shortest and smoothest path without stopping, whereas vehicles accelerate or decelerate without changing their trajectory. (Pascucci 2015) These results are consistent with what was found in the Rudloff et al. study as well. This knowledge was then taken to form a new layered structure for the simulation model.

In this new model, only three layers based on social force model were utilized to determine a user's movement. These levels were the free flow trajectory (FFT), long range collision avoidance (LRCA), and short range collision avoidance (SRCA). As in previous 
models, the FFT level creates the most desirable path for that user without the interference of other users. The collision avoidance layers employed a new method of decision making by taking four steps. First, an opposing user must be perceived, then the conflict detected and distance to that point calculated, followed by a classification of the conflict avoidance, and finally the reaction. (Pascucci 2015) This model in particular uses the social force model for the SRCA, but for the LRCA simply tells pedestrians to choose a new FFT in a pedestrian-pedestrian conflict, and if a vehicle is involved in a potential conflict their reaction will be to change speeds. Further research in to this model is needed in the future to continue testing the validity and calibration of the parameters.

\subsubsection{Viswalk and Vissim combination}

Another unique way to simulate the interaction between vehicles and pedestrians was created by consultant $\mathrm{CH} 2 \mathrm{M}$ in cooperation with software developer PTV. This method combines the capabilities of the industry standard PTV Vissim software with a relatively new pedestrian specific software PTV Viswalk. In essence, by combining these two software packages, the social force model parameters used in Vissim can be applied to interactions between multi-modal users instead of simply single mode users. In this specific case, vehicles were represented by groups of pedestrians that moved as one single unit, the equivalent size of a vehicle. Even though in typical Vissim simulations, vehicles and pedestrians are limited to interactions via rule based decisions, and do not "see" each other, by making the vehicles actually be pedestrians they can then interact. (Gibb 2015) This method, referred to as dummy pedestrians, tricks the system in to thinking a single car or bus is actually a set of pedestrians walking together in the dynamic fashion to coincide with that of a vehicle's characteristics. This 
workaround captures the basic dynamics of real life interactions between users very well. Some tendencies of pedestrian to predict vehicle paths or avoid crossing in front of larger vehicles is lost however in this method as all users are seen as pedestrians.

This modelling strategy also brought about a quantitative prediction relationship between the volume of vehicles and crossing pedestrians to determine the capacity of individual shared spaces. In this case, a standard 60 meter long shared space was used with varying amounts of pedestrian crossing volumes and vehicle through traffic. Theoretical breaking points were determined from these multiple runs, with which a table and graph could be drawn. (Gibb 2015) This simple relationship can be extremely helpful in the future on projects as a first test for proposed intersections. The vehicle and pedestrian crossing demands should be able to be determined easily by local authorities or consultants, and whether or not there will be sufficient capacity can be tested.

\subsection{Conclusions}

Through this literature review, it can be seen how shared space has and can be successfully implemented in multiple different scenarios to improve conditions at intersections and straight corridors of streets. The safety, traffic, economic, and social benefits have been quantified and studied with sufficient detail to promote shared space as a viable alternative. We have noted that limited research in to how users with disabilities should safely navigate a shared space is available, and presents the possibility for future shared space research. Current industry standard social force models have begun to be adjusted and formatted to fit the unique dynamic interactions between users of pedestrians to simulate their behaviors closely. Although these models represent with great accuracy the individual interactions between pedestrians, they are currently lacking in measurement of traffic metric measurements such as delay, travel time, and 
level of service. The application of these models to the analysis of multiple different alternatives including non-shared space designs is inhibited by the specific behavioral models used unique to shared space. A modeling technique which can capture the shared space dynamics, while also being available to simulate more traditional designs within a small network are needed in the industry to fill the chasm of technology between our current abilities and what is required to advance shared space as a viable alternative to planners and engineers. 


\section{RESEARCH METHODOLOGY}

This methodology will begin by examining the basic assumptions made and strategies taken to simulate a shared space environment using a PTV Vissim model. Fitting the model to the case specific criteria will then be described in detail. Finally, the method of data collection will be outlined.

\subsection{Creating the PTV Vissim Model}

The research objective to build a microsimulation model capable of evaluating traffic effects of implementing shared space was begun by determining the appropriate software, technology, and approach. As demonstrated in the literature review, there are current models which have been created to effectively simulate the interaction between a vehicle and pedestrian in a shared space environment. However, these models are currently private innovations and have not been scaled up to encompass an entire case study area in order to estimate traffic parameters such as delay. Although the methodology used to create said models could be replicated and perhaps expanded in the future to be larger and able to measure traffic parameters, it was deemed to be too complex for widespread use in the industry. Instead, it was decided to take a current software common to the transportation planning and engineering industry and adapt it to be able to simulate shared space as closely as possible. This would save engineers and planners time and the need to develop their own models from scratch. With this in mind, PTV Vissim was chosen to be the platform for this model due to its availability, industry prevalence, reputation, and flexibility. 


\subsubsection{Adapting PTV Vissim to Shared Space}

PTV Vissim software comes with many default settings that properly replicate a traditional roadway environment. The program allows the engineer or planner however to adjust these to more closely fit the selected location of study. In the case of this research, this was an integral part to being able to use PTV Vissim to simulate a shared space alternative.

The focus of the adaptations made within the model were to change the way in which pedestrians and vehicles interacted, as well as where they could travel. The second goal to change where the vehicles and pedestrians could travel involved a rather simple change that is done frequently within Vissim which is to relocate the respective vehicular and pedestrian links in the model. The first task however is more complicated since all other types of traffic design have very defined traffic rules and definition of priority. As has been expressed, this is the opposite of the principle with shared space designs.

Within PTV Vissim, the current standard to determine right of way between vehicles, pedestrians, or vehicles and pedestrians is by using conflict areas or priority rules. Both methods allow the user to dictate which direction of flow has priority over the other. The flow without priority will then yield to the other movement of traffic. There is also the option of not placing any rules regarding priority in the simulation which results in simulated users not seeing each other and behaving as such. The last option exists only within the conflict area tool, and allows one to express to the simulated users that there is a potential conflict but that it is not defined. In this case simulated users can see the other users that their movement will conflict with, and are then left to their own devices to decide which user shall proceed first. In the model this is determined by multiple metrics that are either measured or randomly assigned to drivers and pedestrians like which user arrived first, vehicle speed, distance away from potential conflict, 
level of driver/pedestrian aggression, etc. These metrics are the same as those used in the social force approach models discussed earlier and the variables that real life shared space users would encounter in order to make a decision within shared space as either a pedestrian or driver. These factors made it the closest and most suitable fit to simulate shared space.

This undetermined conflict area tool however was found to only be accurate between two vehicles, or two pedestrians, but not between a single vehicle and single pedestrian. When this undetermined priority was placed between a single vehicle and single pedestrian the users behaved nearly identically as if the pedestrian was given priority. In the literature review, it was noted in previous models and data collected from the field that shared space interactions between vehicles and pedestrians can be summarized by vehicles staying course and only accelerating or decelerating to avoid collisions while pedestrians perform the opposite behavior and vary their route to avoid collisions but remain at a constant speed. Since pedestrians are unable in PTV Vissim to stray from their link's path, and the vehicles would always yield to them, it was deemed that this undetermined conflict area was the appropriate and conservative option when attempting to estimate the traffic delay, but could also be replicated with the pedestrians having outright priority and resulting in similar outcomes.

Finally, the lane/link width for vehicles was reduced to $6 \mathrm{ft}$. in order to reduce the distance between pedestrians and vehicles to trigger a conflict. This allows for vehicles in the model to only yield to pedestrians when a collision will occur, rather than yielding to all pedestrians in the lane or crosswalk even when a collision will not occur. The result is a smaller headway between vehicles and pedestrians as exists in real world shared space scenarios. Likewise the pedestrian links were also reduced in width to represent the width of 1-2 pedestrians crossing rather than an entire width of a crosswalk, therefore better representing the space they actually occupy. 


\subsubsection{Designating and Spacing Pedestrian Routes}

Theoretically in a shared space environment, pedestrians are free to cross the street in whichever path they desire. This lack of designated crossing points leads to an infinite number of O-D pairs and crossing points that would need to be simulated in the model. Knowing however that pedestrians will ordinarily take the shortest path possible, we can reduce the number of crossing points necessary to include in the model by identifying major origins and destinations, and mapping the crossing paths between them. Research has shown that in a real shared space scenario, vehicle, bicyclist, and pedestrian routes tend to cluster around a limited number of crossing paths as shown in Figure 11.

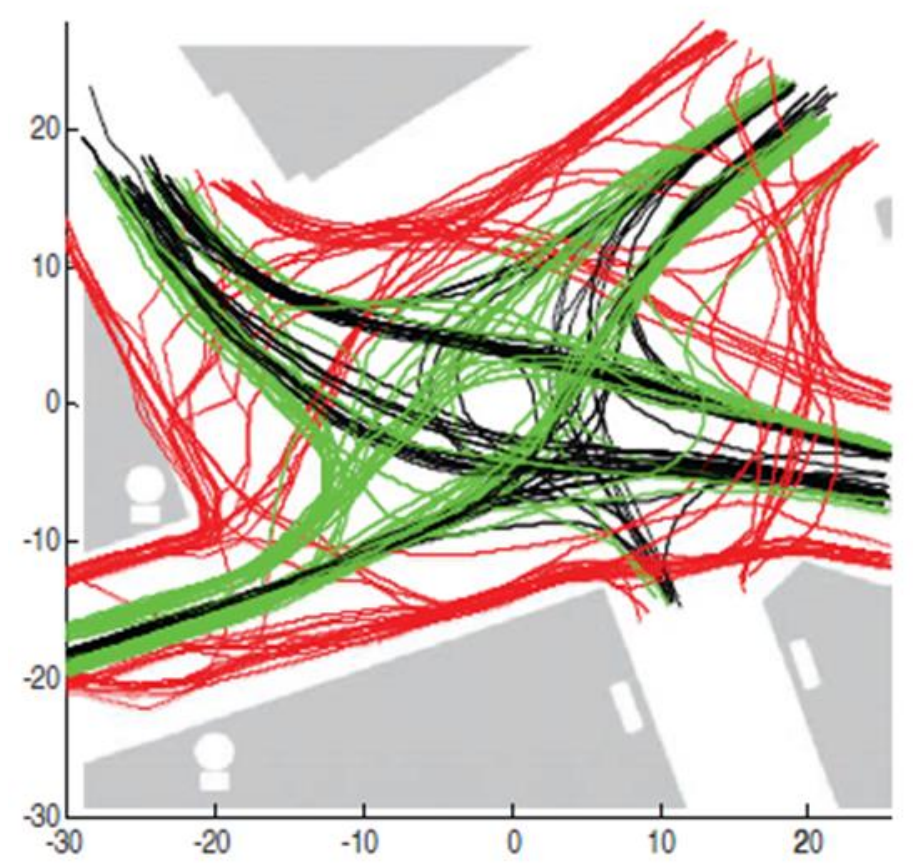

Figure 11: Observed vehicle, bicyclist, and pedestrian paths in Graz, Austria (Rudloff 2013)

In the above figure, the green and black lines represent travel paths taken by vehicles and bicyclists respectively, followed by the pedestrian travel paths in red. We can see that the pedestrian paths are the most diverse crossing paths but can still be summarized in a handful of 
O-D pairs and routes. This knowledge allows the modeler to choose how many pedestrian crossing paths, and where to place them, in the PTV Vissim model based off of knowledge of current pedestrian trip generators. The number of paths required to simulate the shared space as closely as possible will vary between sites based off of the surrounding environment. Keep in mind that the pedestrian routes chosen to model should represent the shortest path for a pedestrian group and will therefore be more likely to cross the space at an acute angle rather than at a perfect ninety degree angle as is typical for designated crosswalks. The combined paths modeled in PTV Vissim should characterize the desired movements of the majority of the pedestrian users.

It should also be noted that the spacing of the pedestrian crossing paths is of critical importance. Due to the setup of the PTV Vissim software, there must be sufficient space on a vehicular travel link between two neighboring pedestrian crossing points to accommodate the largest class of vehicle being modeled. Innately, PTV Vissim does not allow a vehicle to cross a pedestrian path (conflict point) until it knows that the vehicle can traverse that conflict point without being stopped at the next one and consequently block the preceding conflict point. Therefore, without enough space for a vehicle to stop between two conflict points, that vehicle must wait until both conflict points are clear. This does not mimic the real workings of a shared space, and therefore it is imperative that there be enough space on each vehicular link between neighboring conflict points for the largest vehicle to stop. This layout of pedestrian crossing paths allows for vehicles to incrementally progress through the intersection as they would in a real shared space. Referring back to Figure 11, this spacing of pedestrian paths also matches up with the arrangement of the natural clusters of pedestrian movements and therefore will simulate shared space behavior effectively. 
In studying the current pedestrian trip generators and O-D pairs at a study location, the volumes of pedestrians on each path during a given time period can be predicted. In this model it was chosen to slightly increase the pedestrian volumes for the future years to accommodate for future growth in population and higher use by pedestrians of the area, consistent with the literature findings. To add resiliency to the model, multiple pedestrian volume and distribution between paths arrangements were simulated against the same vehicular volumes to show whether or not the shared space could adapt to varying degrees of pedestrian volumes and distributions of O-D pairs.

\subsubsection{Additional shared space modeling assumptions}

Another main change which was applied in comparison to a traditional traffic model in Vissim was the vehicular speeds. In this case, our research points us to the fact that shared space designs even without the use of posted speed limits will reduce observed vehicle speeds to the range of 10-20 mph. Depending again on the location and dynamic aspects of vehicle and

pedestrian volume, the speed at any given shared space can vary between these two ranges. Since this model will be used to test the congestion relief aspects of shared space, the more conservative value of $10 \mathrm{mph}$ was chosen.

\subsection{Case Study Simulation}

The case study location which was selected is located on the Downtown campus of West Virginia University in Morgantown, WV. WVU is currently home to approximately 30,000 students and another 30,000 citizens within the city limits. There are two main arteries, Beechurst and University Avenues, which run in the general North-South direction within the Downtown campus. University Avenue bisects the Downtown campus of WVU with many of 
the freshman dorm facilities as well as the student union, known as the Mountainlair, on one side and the majority of the academic buildings on the opposite side of the street. Therefore a pedestrian crosswalk was necessitated and in the 1930's a pedestrian island and single unsignalized crosswalk were installed at this location under the direction of then facilities manager and professor Dr. Grumbein to facilitate the safe crossing of students, faculty, and citizens. As the student population has greatly increased over the past 80 years, this location now known as Grumbein's island, experiences daily congestion and traffic delays for drivers on University Avenue.

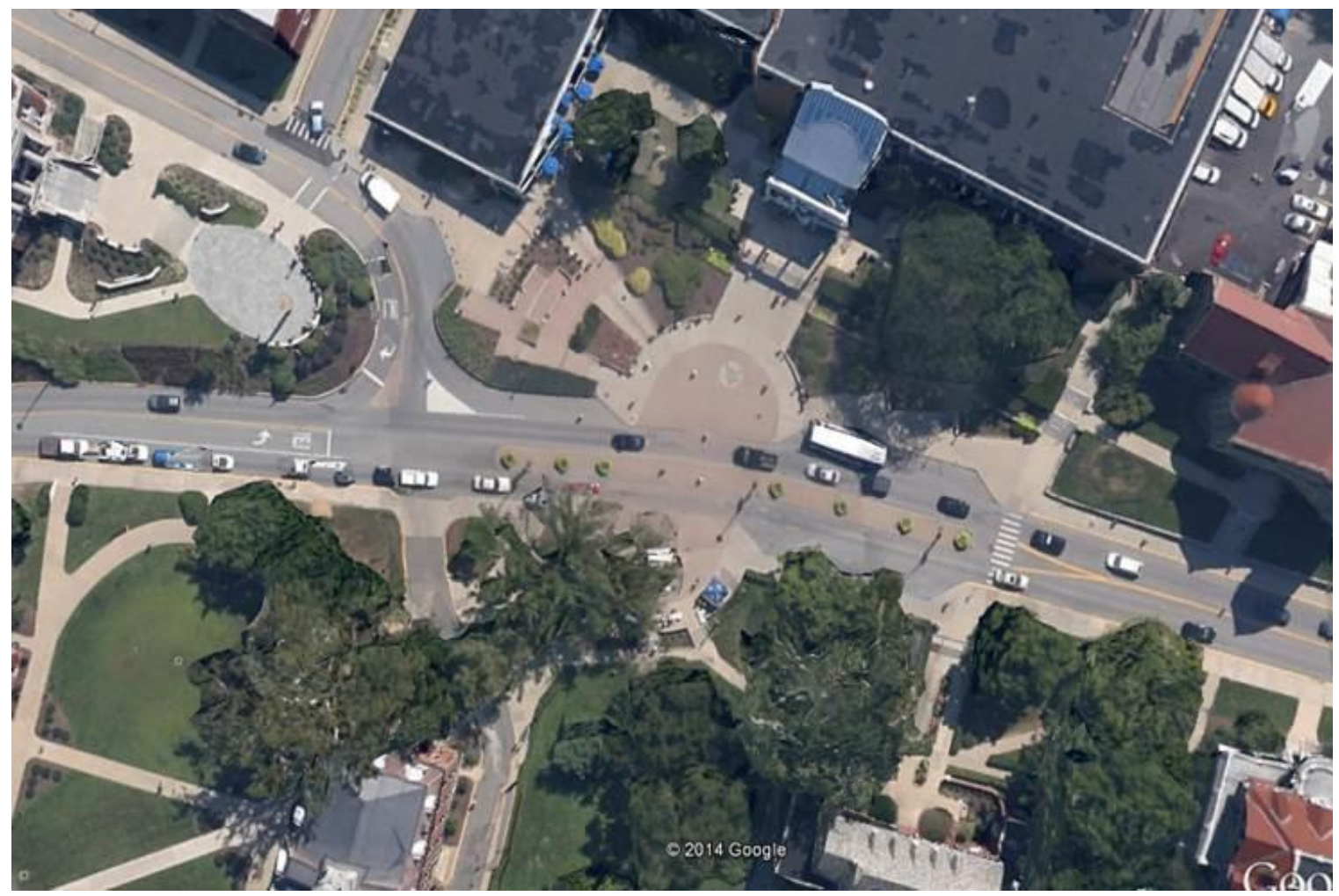

Figure 12: Current Grumbein's Island Configuration on University Avenue in Morgantown, WV (Google) The arrangement of the WVU facilities with one main "crosswalk" on University Avenue results in a large number of pedestrians crossing University Avenue directly in front of the Mountainlair for a 10-20 minute period between classes every hour on Monday, Wednesday, and 
Fridays, and every approximately hour and a half on Tuesday and Thursdays. The influx of pedestrians to a single un-signalized crosswalk causes drivers to need to stop and wait for an extended period of time as the headway between pedestrians is typically insufficient to drive through. Long vehicle queues begin to form rapidly at this crosswalk as the rate of vehicles entering the queue from other streets is much greater than the rate at which cars can cross this single crosswalk. After the approximately 20 minute period is over, the queue begins to recede until normal traffic flow is resumed an additional 10-20 minutes later. This means that at multiple times during the day, there is an almost 40 minute period every hour in which traffic on this street is backed up, moving slowly, or potentially stopped for an extended period of time.

In the past, bridge or tunneling projects have been suggested with significant price tags and implications for accessibility on the campus and have therefore never been fully pursued. In 2013 a re-routing of University Avenue behind the Mountainlair was proposed by representatives of the Student Government Association of WVU. It was at this point that this research team began proposing shared space as a potential solution and turned to simulation techniques to model the improved traffic capabilities of this alternative design. The ability of shared space to allow users to choose their shortest path will spread out a current single stream of pedestrians in to multiple paths of pedestrians crossing a single arterial street at varying locations. This will theoretically increase the headway between two individual pedestrians, therefore allowing vehicles to continue gradually traversing the area. 

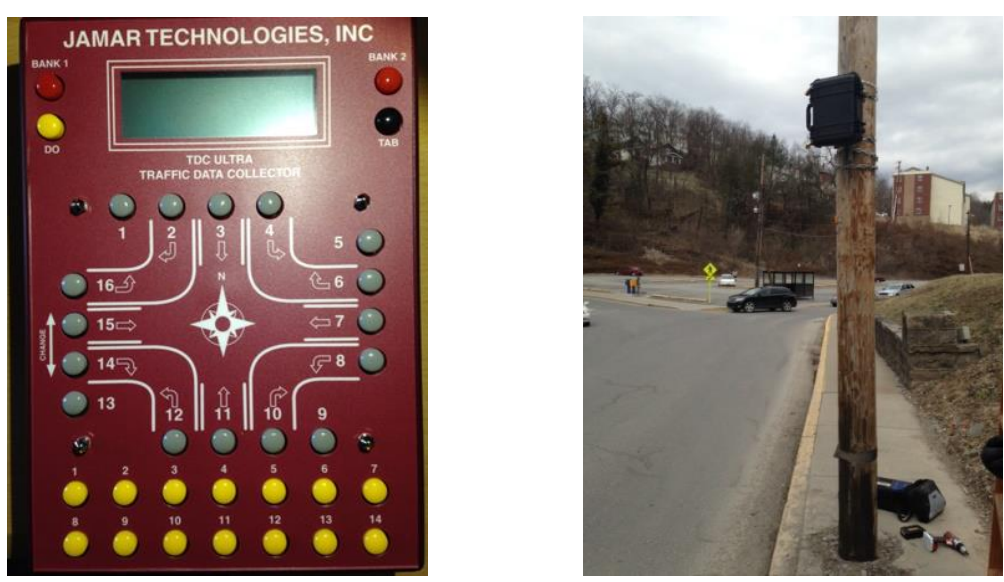

Figure 13: Data Collection Devices (Left: manual turning counter, Right: mounted radar vehicle counter) During the weeks of March $23^{\text {rd }}-30$ th, and March $30^{\text {th }}$-April $6^{\text {th }}, 2014$, a two-week-long data collection period was undertaken to provide base data to create a model of the current scenario and base the parameters for the shared space model off of. Mounted radar vehicle counters and manual turning counters, operated by a group of volunteers, as shown in Figure 13 were used to collect the data during this collection period at the locations shown in Figure 14.

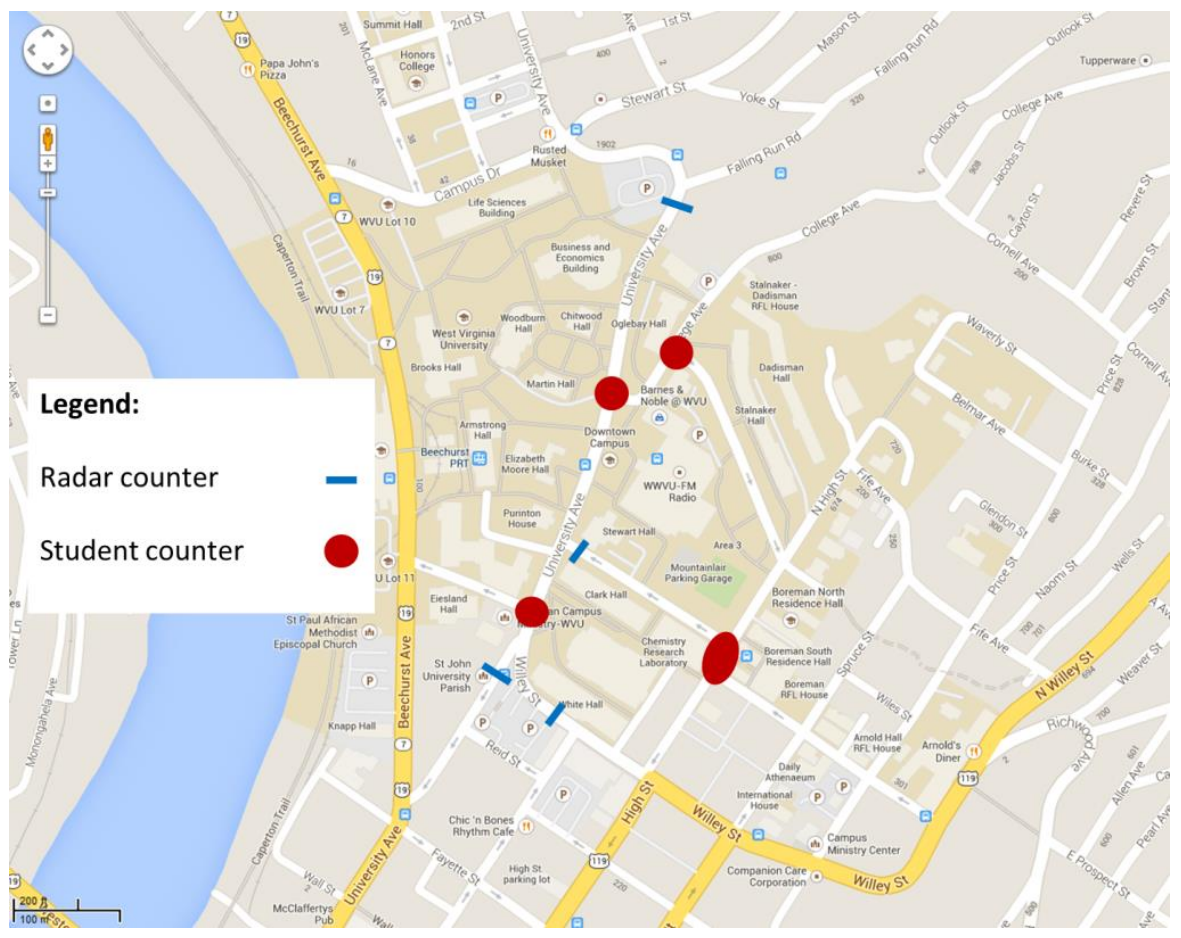

Figure 14: Radar and Manual Counter Data Collection Locations (Google Maps) 
Figure 15 presents a summary of the data collected during this first data collection period, which included vehicle volumes during peak and non-peak hours, vehicle turning ratios at intersections, as well as pedestrian volumes on a 15-minute time interval. This out of the ordinary pedestrian volume time period was chosen to capture the unique volume changes over the course of time at a university campus. The simulation model utilized the PM peak hour vehicle volumes.

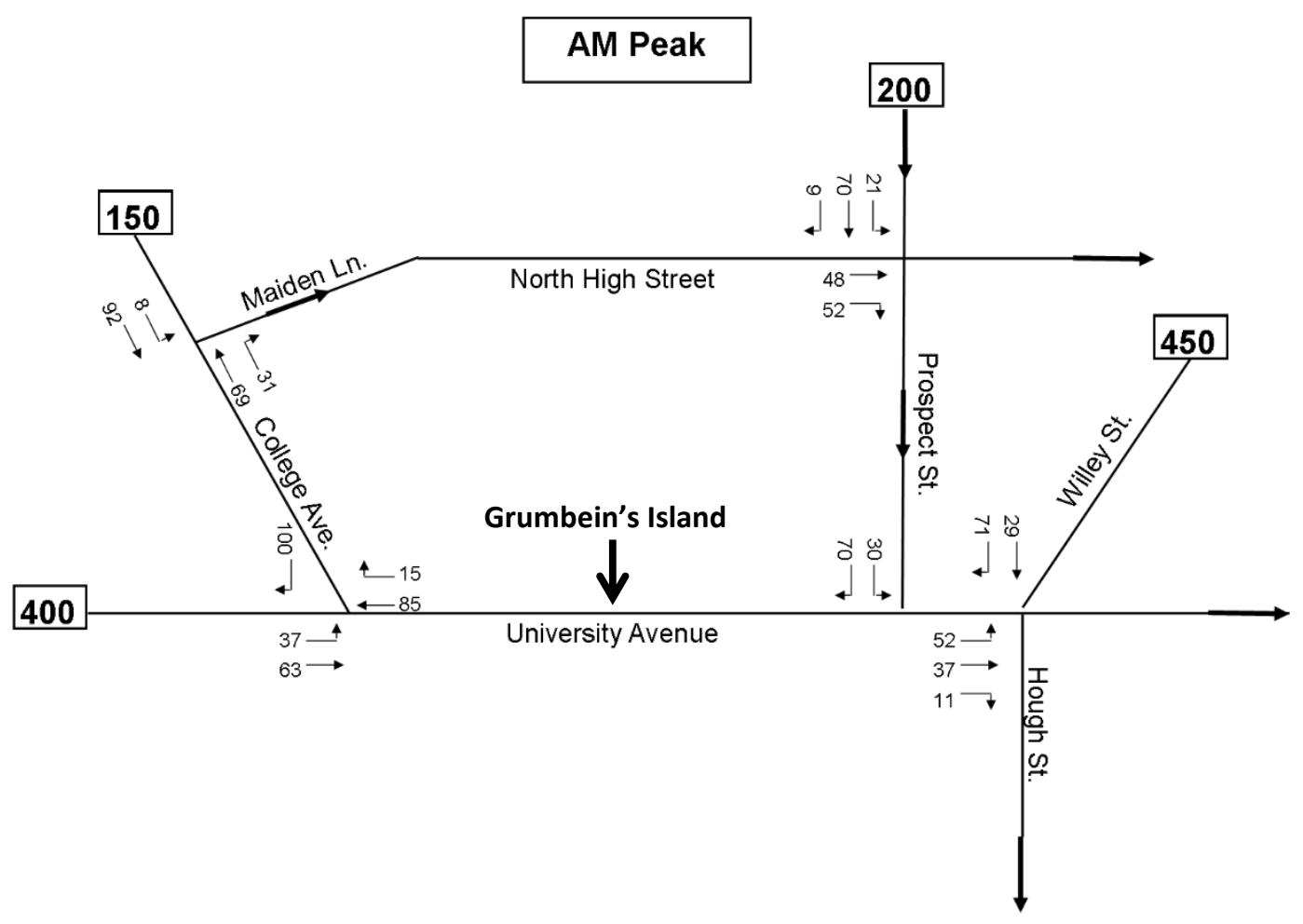

Figure 15: Morning One-Hour Peak Vehicular Volume at Case Study Location 
Frosch 49

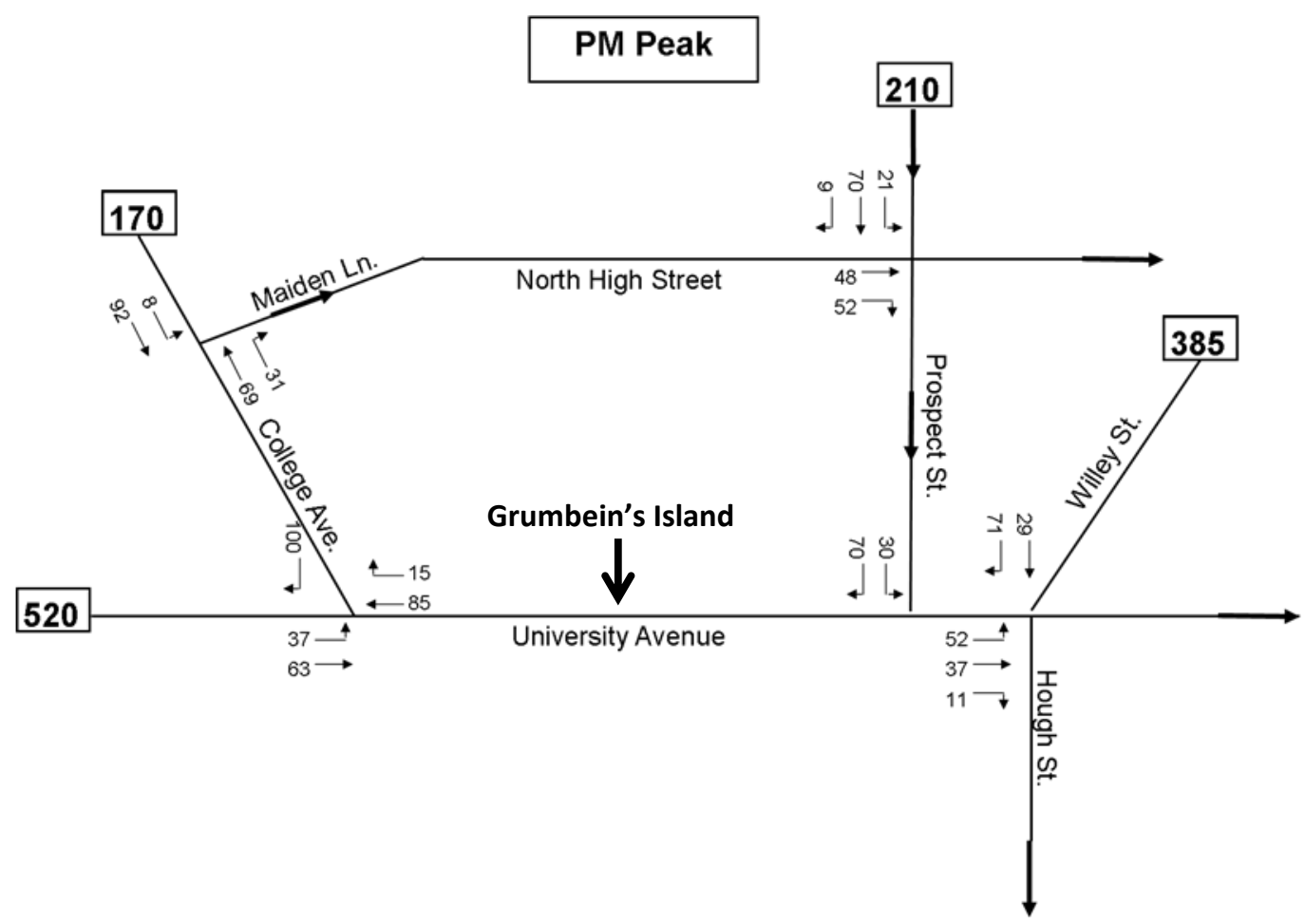

Figure 16: Evening Peak Vehicular Volume at Case Study Location

Pedestrians Non-Peak (15 minute increment)

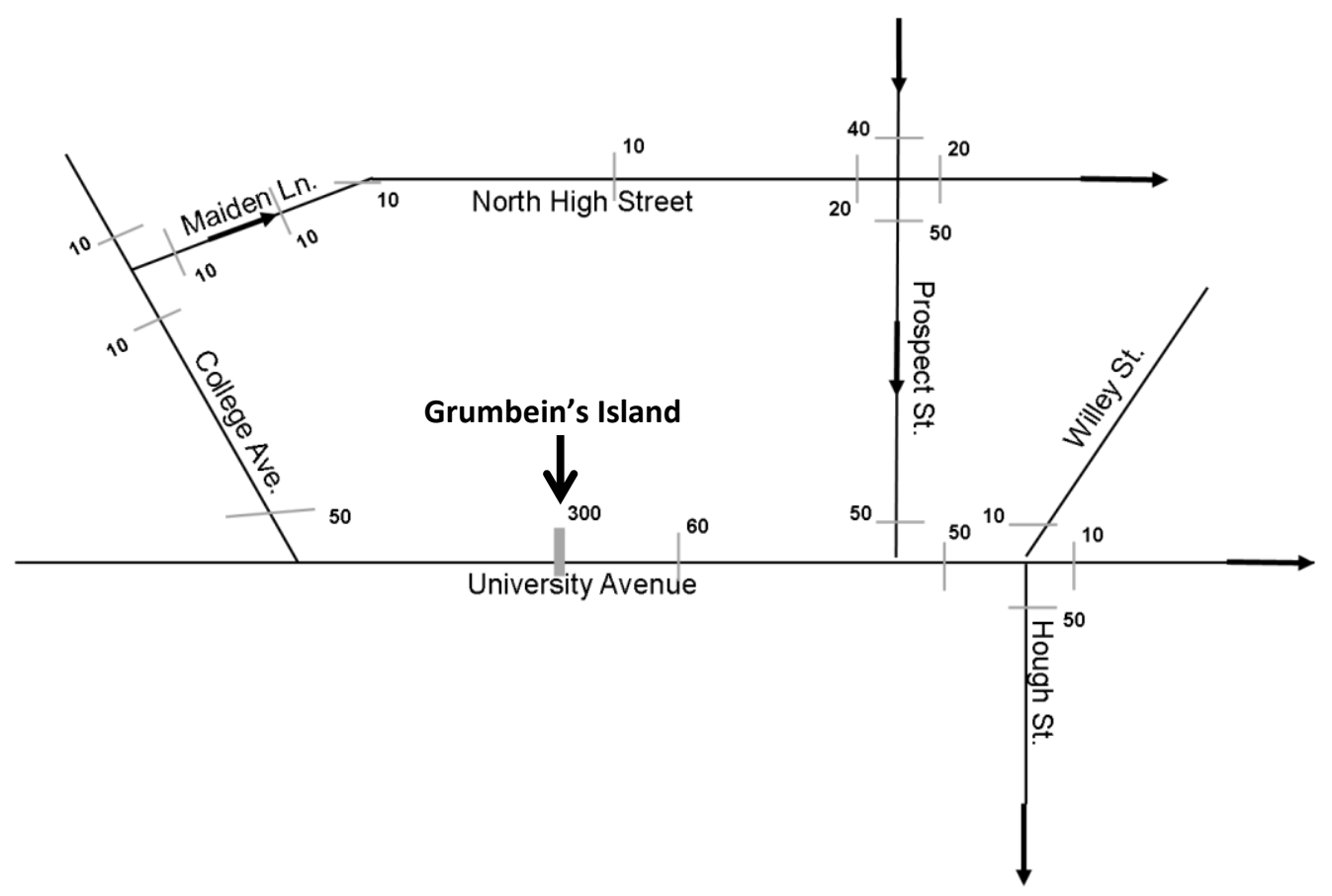

Figure 17: Pedestrian Crossing Volume During Non-Peak 15 Minute Increment 
Frosch 50

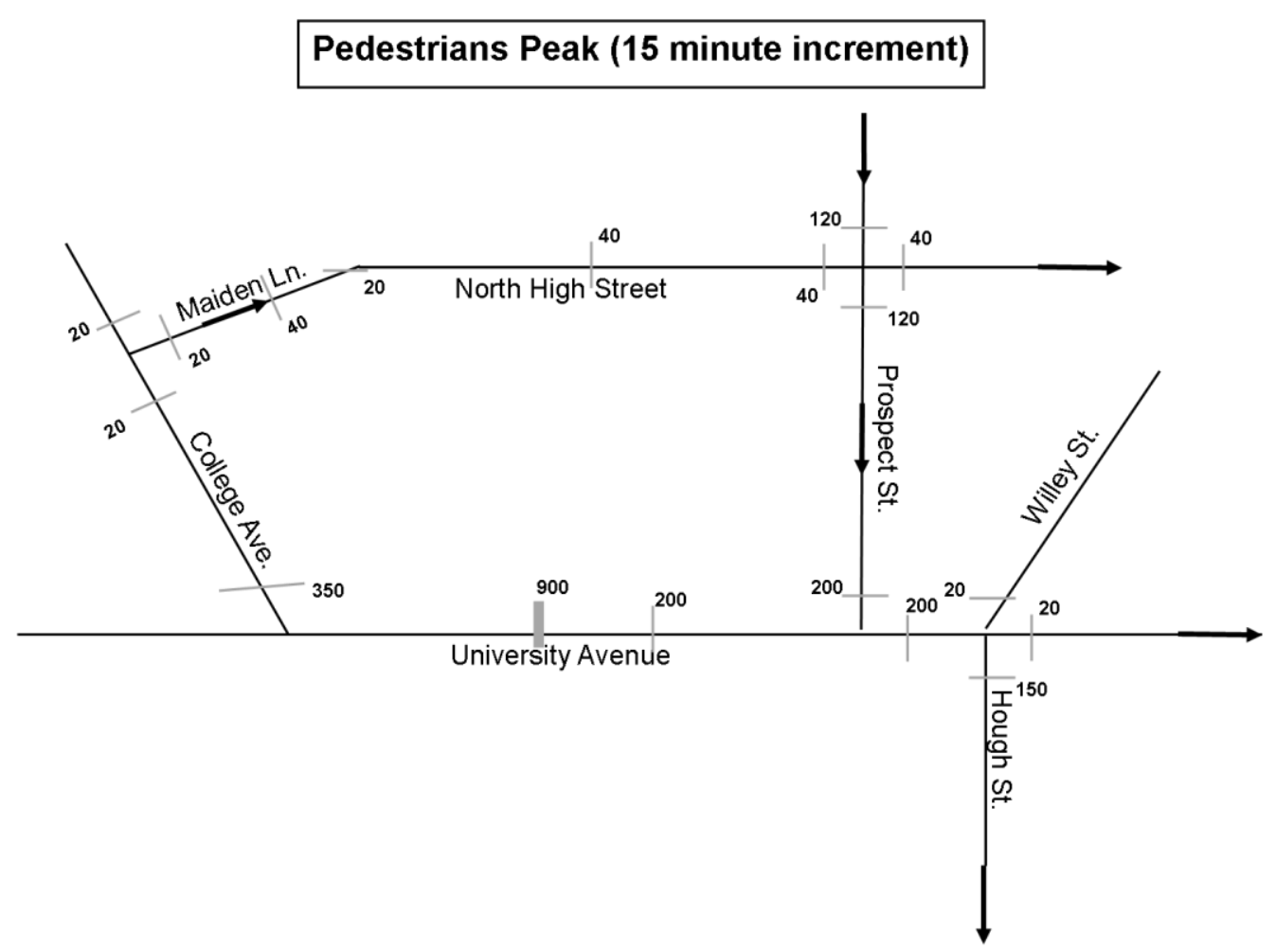

Figure 18: Pedestrian Crossing Volume During Peak 15 Minute Increment

Another data collection period was also performed on April $27^{\text {th }}, 2015$ to focus solely on the pedestrian volumes crossing at the Grumbein's island location. The results can be seen below in Table 1. Unlike the previous data collected, this collection was performed in one minute increments to gather a better microscopic understanding of the pedestrian flows. The 2015 values coincided with the 2014 values as they were on the same order of magnitude. A visual representation of the pedestrian flows is provided in Figure 19.

Table 1: Pedestrian Data Collection 2015 Summary Values

\begin{tabular}{|c|c|c|c|c|c|c|}
\cline { 2 - 7 } \multicolumn{1}{c|}{} & \multicolumn{3}{c|}{ Peak (X:10-X:29) } & \multicolumn{3}{c|}{ Non-Peak (X:30-X:09) } \\
\hline Origin & Beechurst PRT & Mountainlair & Sum & Beechurst PRT & Mountainlair & Sum \\
\hline Mean & 24.86 & 21.69 & 46.55 & 9.32 & 7.96 & 17.28 \\
Variance & 24.86 & 21.69 & 46.55 & 9.32 & 7.96 & 17.28 \\
Standard Deviation (Poisson) & 4.99 & 4.66 & 6.82 & 3.05 & 2.82 & 4.16 \\
Standard Deviation (Sample) & 13.71 & 10.07 & 18.67 & 5.92 & 4.03 & 7.44 \\
\hline Minimum & 6 & 4 & 18 & 1 & 0 & 5 \\
\hline Maximum & 67 & 51 & 86 & 41 & 23 & 51 \\
\hline
\end{tabular}


Once the PTV Vissim model was created for the current configuration, and travel time values were measured in the model, in-person trial travel time runs were taken to verify the model. This verification process was a success and warranted further progress on the model to now include the shared space design. Further results of the current configuration travel time and delay values can be seen in the results section.

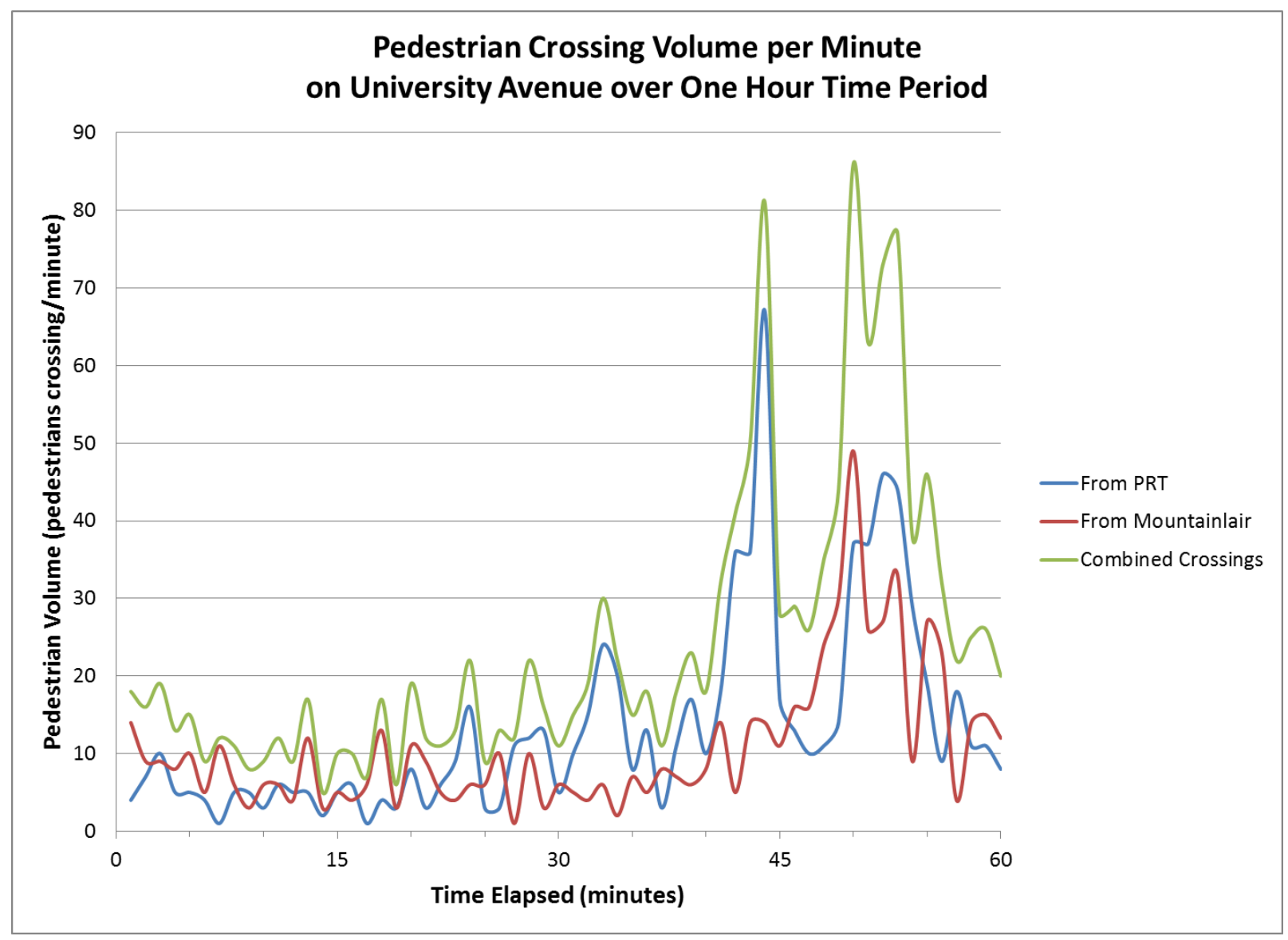

Figure 19: Pedestrian Crossing Volume per Minute on University Avenue over One Hour Time Period

\subsubsection{Fitting the Model to the Study Location}

In order to best fit the proposed shared space design to the modeling parameters described above, it was first necessary to map out the pedestrian O-D pairs that would be naturally occurring in this area. As discussed previously, the pedestrians will be motivated to 
take the shortest path possible, a straight line, between their origin and destination once they've entered the shared space domain. To facilitate this, major origin and destination locations were identified in the near vicinity of the shared space boundaries. In the case of WVU's campus this correlated to mainly academic and student service facilities. Pedestrian routes were then transcribed on to a satellite image connecting these designated origins and destinations. Pedestrian routes first followed sidewalks and pathways to get to the approximate boundary of the shared space and then make a single straight line to the chosen destination. The resulting web of O-D pedestrian pairs is shown below in Figure 20. As can be noted in the figure, only two of these identified routes are centrally located to cross at the existing Grumbein's island location. The remaining pedestrian crossings of University Avenue are instead spread out throughout the shared space area. It should also be noted that many of these crossings are at an angle other than perpendicular to the flow of traffic, as would be typical at a designated cross walk. These routes also assume that some landscaping would be removed to allow better access to the shared space.

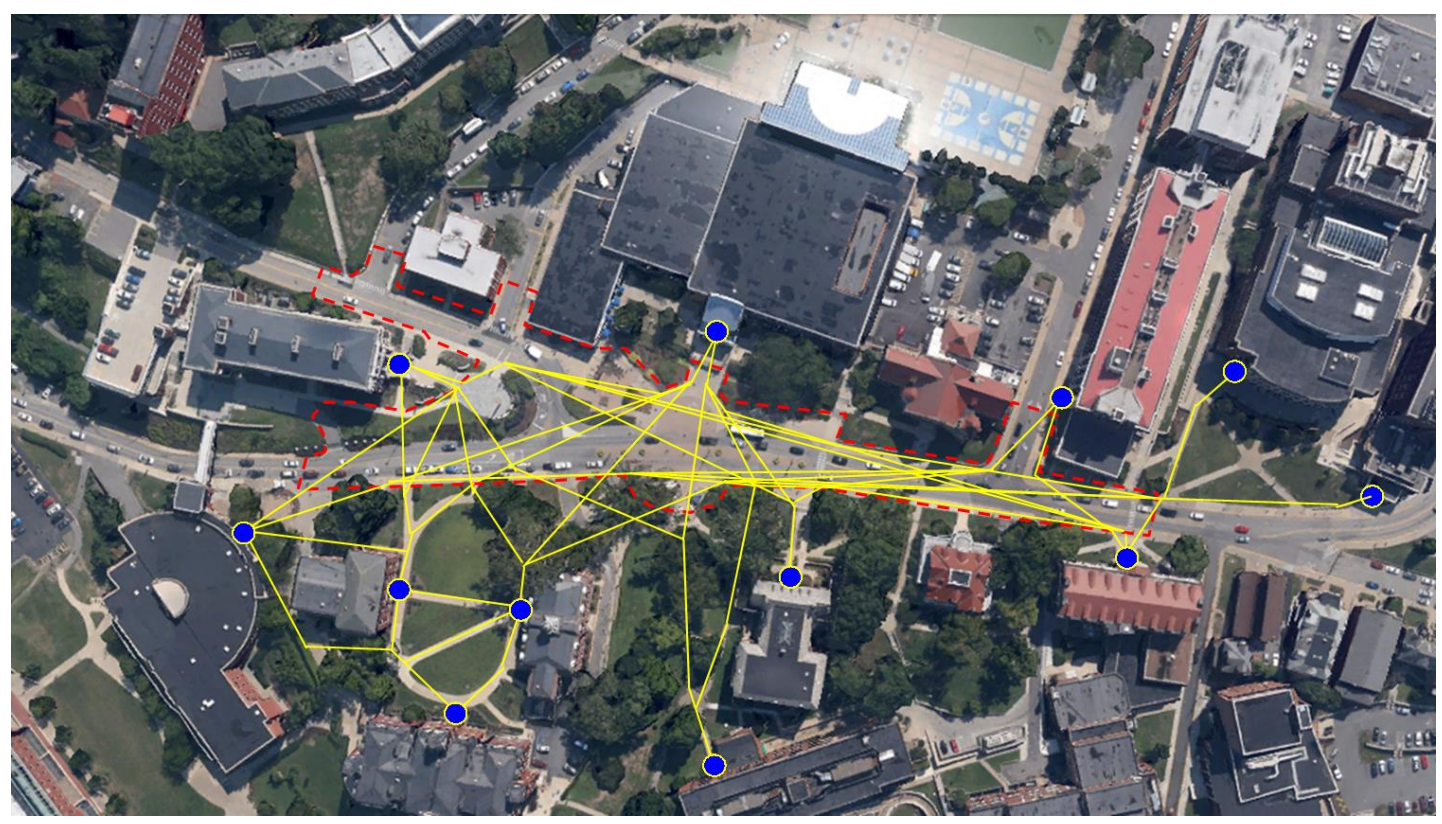

Figure 20: Forecasted Pedestrian O-D Pairs (Blue: Origin/Destination, Yellow: Routes, Red: Shared Space Boundary) (Google) 
Now that these pedestrian paths had been identified, they could be used to guide the design and layout of pedestrian links in the PTV Vissim model. As mentioned earlier, the spacing of these pedestrian links is critical to making the model work correctly. By assuming that all of the pedestrians will take one of the identified routes, rather than one of an infinite combination of routes, a conservative estimate for the impact they will have on the traffic congestion in the area will be made. With a constant level of pedestrians crossing the street, a larger number of pedestrian crossing route options will increase the headway between crossing pedestrians on any given path and provide more opportunities for vehicles to flow through. By limiting the number of crossing paths, the headway between pedestrians will not be increased to the maximum level; rather a more conservative estimate of the headway will be achieved.

In collaboration with WVU administrators and Stantec Consulting Services Inc. a conceptual design rendering was created, and is shown in Figure 21 below. This design kept many of the shared space principles as cornerstones but was also context sensitive to the use and layout of the area. Some of the key design aspects that should be noted are the varying colors and textures of pavement, addition of sidewalks and stairs to increase points of access to the shared space, as well as removal of curbs, striping, and other pavement markings. This conceptual design also enabled the team to devise a best estimate of the design and construction for this project of approximately $\$ 4$ million dollars.

Although the pedestrian only areas and vehicle travel ways will be at the same elevation, there will be a distinct difference based off of the pavement texture and color. Metal or concrete bollards will also be placed sparingly at specific locations along the perimeter of the shared space to provide safe pedestrian only areas. Varying colors of pavement were also designated in areas where major vehicle-vehicle conflict points would arise or where a significant volume of 
pedestrians would be crossing. These will serve as an additional "warning" to alert users to be more aware of potential conflicts in these areas.

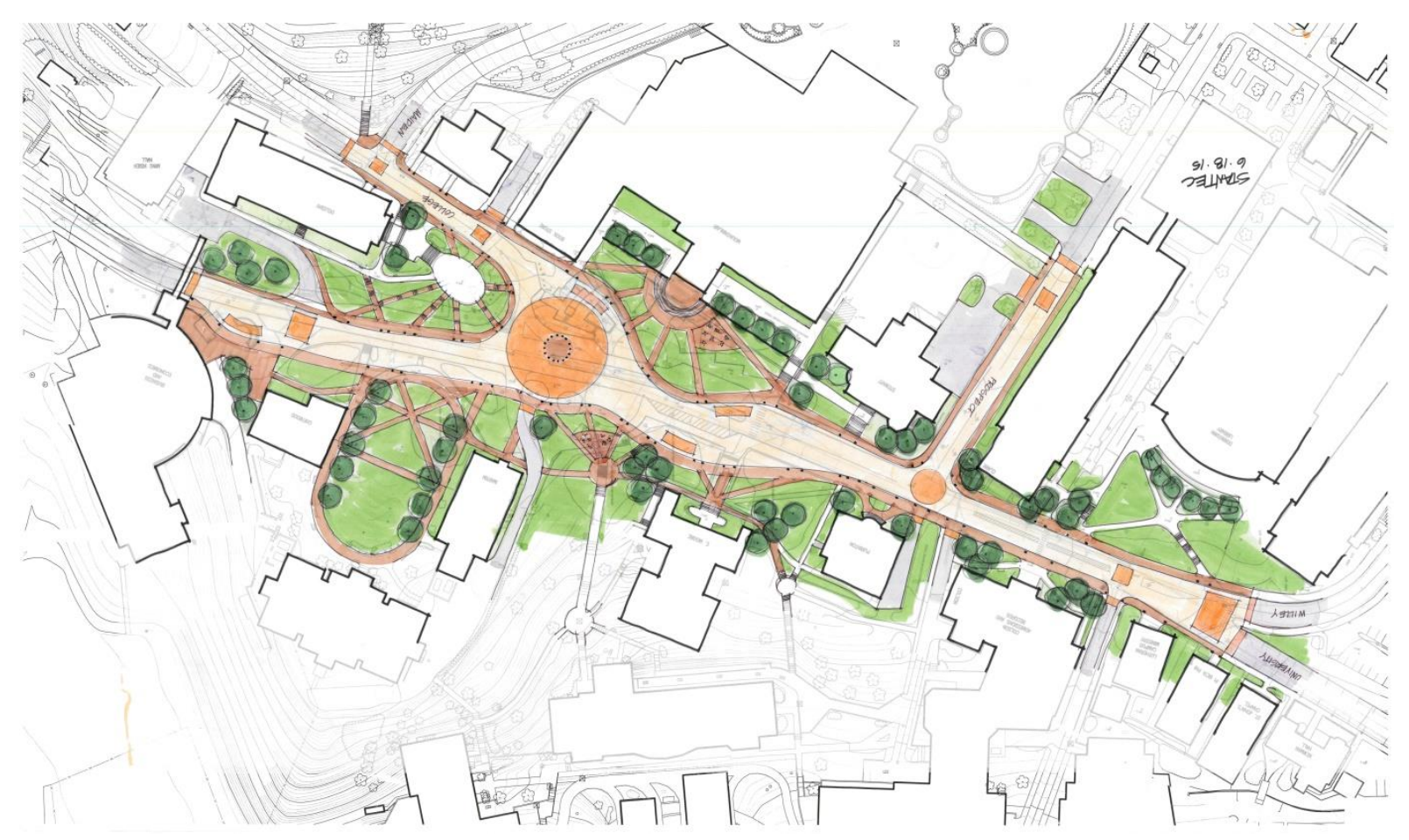

Figure 21: Shared Space Conceptual Design (Stantec)

One of the key concepts that became a focus of this design was the transition zones for both vehicles and pedestrians in to and out of the shared space. The transition zones needed to be sufficient in order to reduce the vehicle speeds to an acceptably safe rate, and raise the driver and pedestrian awareness prior to entering the shared space. For drivers, this meant a vertical element near the entrance of the shared space, a change in pavement texture and color, a slight rise in elevation, and finally the opening of the horizontal area with the removal of curbs and addition of bollards. The same applies for the pedestrians who will transition through a similar three step process of marked by a secluded pedestrian only walkway, which opens to the boundary of the shared space but still protected by bollards, and finally a change of pavement as a pedestrian steps beyond the bollards. This transition improves the safe and efficient operation of the space. 
As was mentioned earlier, a key part of making this specific shared space a success is the ability of pedestrians to spread their desired routes out over the entire area. With the current points of access to the proposed shared space the diversity of paths would be limited. To combat this problem, additional points of access via new pedestrian paths and stairs were incorporated in to this design. With these in place, the amount of total routes will be able to increase and the volume of pedestrians on each route will decrease, increasing the headway between pedestrians as desired. The final consideration which was made for the pedestrians was the implementation of a small pedestrian refuge inside the widest part of the shared space. This was included following concerns for the long distance that would be traversed by a pedestrian at this location.

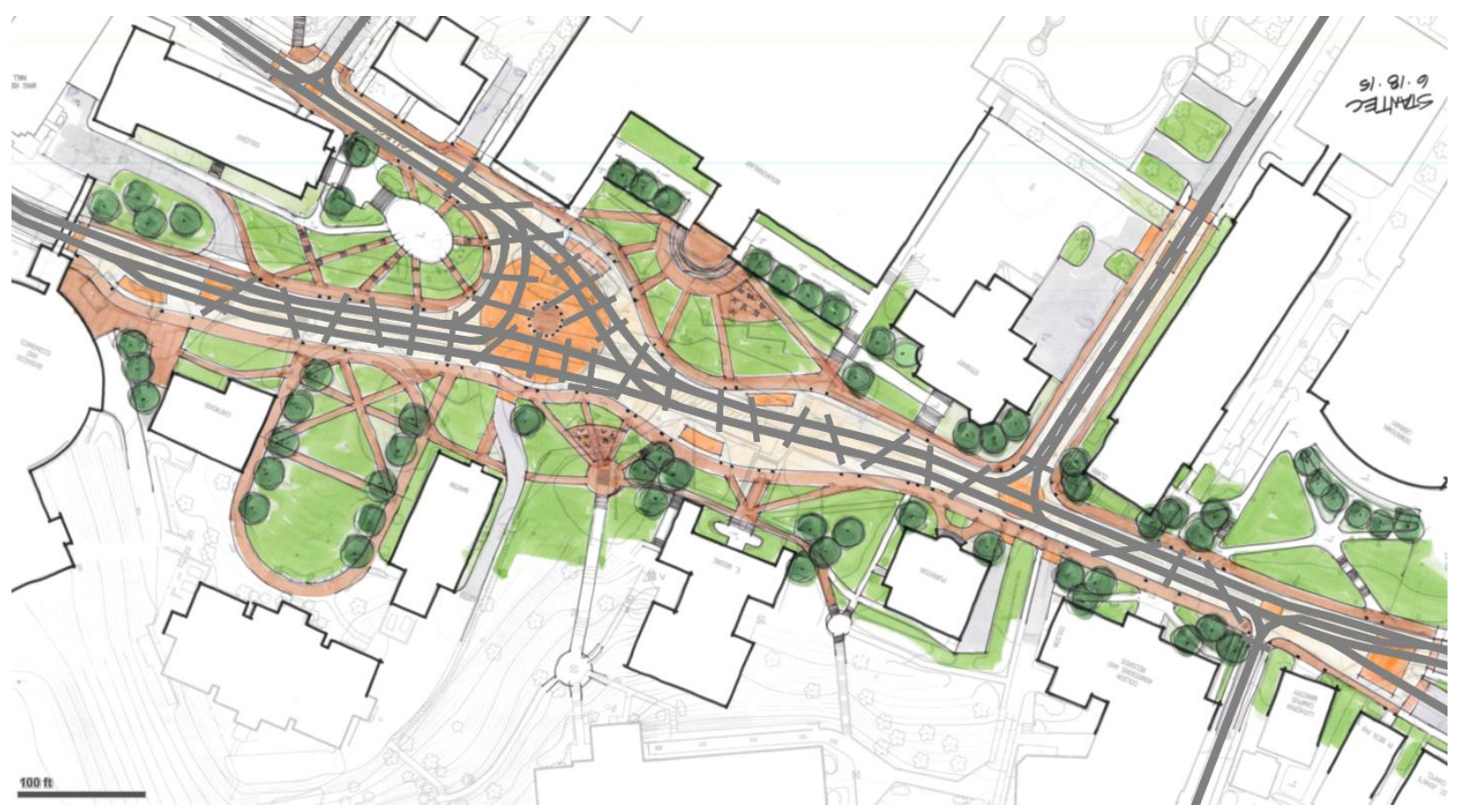

Figure 22: Pedestrian Crossing and Vehicular Links in PTV Vissim Simulation

Once the conceptual design was revised to a satisfactory point by the committee, it was placed in the PTV Vissim software as the foundation for the geometric layout of the model, seen above in Figure 24, including the vehicle and pedestrian links. Figure 23 below shows the 
configuration and designation of conflict areas along the vehicle and pedestrian links. The undetermined conflict areas are designated in red for users in both directions. It can be seen in the figure the multiple conflict areas that are present now that pedestrians are dispersed more. This correlates to a decrease in conflict frequency at each location since the volume of pedestrians and vehicles remains the same.

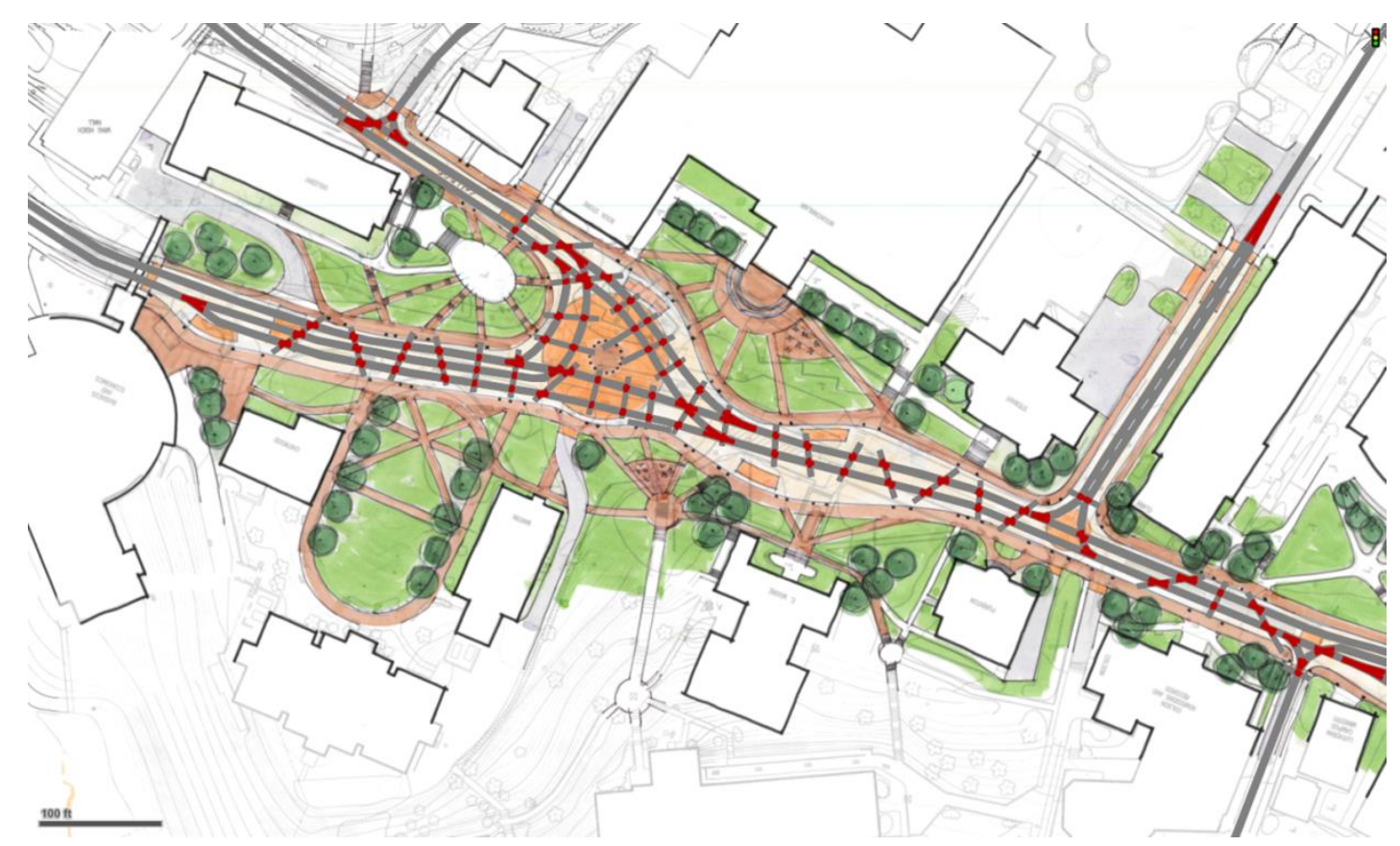

Figure 23: Designated Conflict Areas in PTV Vissim Simulation

\subsubsection{Designing Resiliency into the Model}

As this model would be used to forecast the traffic impacts of the shared space design, resiliency was included in the design of the testing in order to ensure that in many scenarios the shared space would still be beneficial. To begin, the overall volume of pedestrians and vehicles was slightly increased on each route corresponding to growth of the university and city population. These total volumes over the course of an hour were then kept consistent throughout every test iteration of the model. 
Resiliency was added by creating multiple different scenarios of pedestrian dispersion for a total of six shared space simulation iterations. First, three different pedestrian volume dispersions over the four 15-minute time periods were created which put varying levels of stress on the shared space design during the peak 15-minute period. The first configuration was based off of data and conclusions made by observing pedestrians on two separate occasions at the site. The second two configurations shifted the peak 15-minute time period intensity slightly. The actual pedestrian volumes utilized in the model can be seen in the Appendix.

The second level of variation was provided by altering the ratio of preferred routes between origins and destinations that pedestrians would take. This varied the individual volume on each pedestrian link. By combining the two sets of scenarios, with three time variations and two route variations respectively, we were able to make a total of six scenarios to test the model.

We don't anticipate that every day, week, or year will see the same pedestrian volumes or behaviors. Therefore it is important to include this variability that creates resiliency in the model. This way our conclusion can be concrete as multiple scenarios which affect the performance of the shared space have now been considered.

\subsection{In-model Data Collection}

Once the simulation models for the current scenario and six shared space scenarios had been completed, data collection tools available in PTV Vissim were utilized to gather sufficient data to make conclusions regarding the congestion relief and project feasibility. As with the rest of the model, the in-program tools were chosen to provide future engineers and planners with easily accessible means of replicating this type of simulation at other locations. Two main purposes existed for collecting data. The first was to analyze the traffic congestion relief capabilities of the shared space concept within the confines of the project boundaries. The 
second purpose was to assess the economic impact that adoption of this project would have on the entire corridor. It should be noted that all of the metrics measured are in terms of vehicular travel time or delay. Impacts to the pedestrians were excluded from this analysis as they were provided outright priority in the model and would experience zero delay. Also, in the current scenario, pedestrian flow is uninhibited, whereas the vehicular traffic experiences long queues and delay. Therefore an analysis of the pedestrian travel flow metrics was unwarranted in this model.

\subsubsection{Assessing Congestion Relief}

The traffic congestion relief capability of the shared space design was measured using travel time, vehicular delay, and queue length. These parameters were all measured for vehicles traveling within the confines of the shared space design along University Avenue as established which was between Clark Hall and the Business and Economics (B\&E) Building.

The travel time measurements only counted vehicles traveling along University Avenue between Clark Hall and the B\&E Building. Data corresponding to the north and south-bound flows of traffic were kept separate in order to make a more exact comparison between the models. It was important to collect information on the total travel-time between the two defined points to show decision makers and users the potential decrease in their commute.

It was also imperative to measure the delay incurred by each vehicle between the two defined points on University Avenue. As mentioned earlier, the modelled speed of vehicles within the shared space was cut in half; therefore the free flow speed of all vehicles through the shared space will be subsequently doubled. Due to this, it was important to not only rely on the travel time statistics but also the delay. The delay time corresponds to the time a vehicle is 
moving slowly in traffic or completely stopped due to congestion. As a driver, this is often correlated in one's mind to experiencing traffic and a negative experience. For example, if two drivers make a 30 minute commute each, but one sat in traffic for 15 minutes, and the other simply drove for 30 minutes it is expected that the first driver would have a more negative outlook on their drive experience. In addition to the delay incurred between these two points, the delay incurred by all vehicles within the network was also measured. This was done to capture not only the effects along University Avenue, but also the entire network.

Finally, the average queue length on a minute interval time period was measured along with the maximum queue length during each 5 minute interval. These queues were measured in both directions beginning at the edge of Grumbein's island as shown in Figure 24.

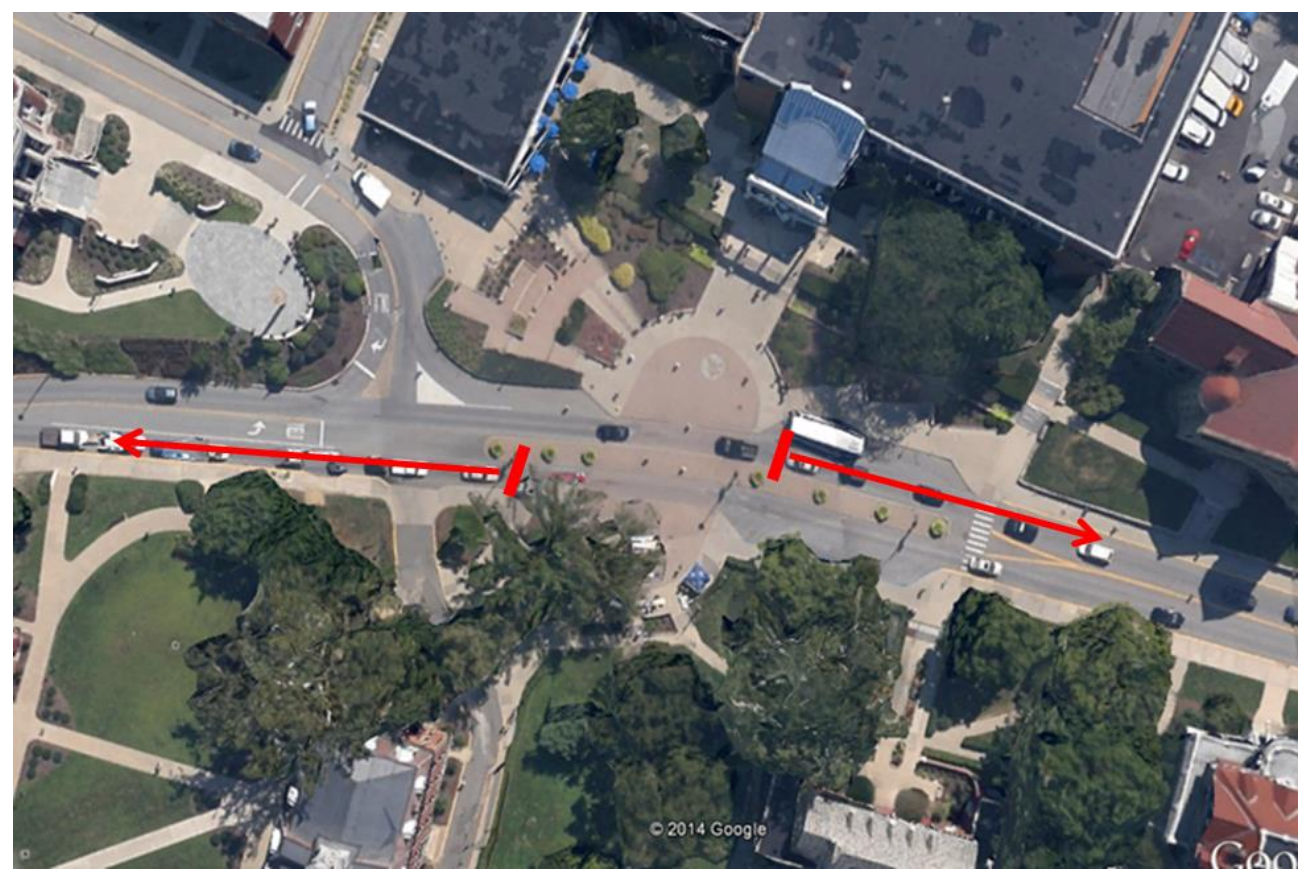

Figure 24: Illustration of Queue Length Measurements beginning at Grumbein's Island

\subsubsection{Measuring Economic Benefits}

In addition to the pure traffic benefits of the shared space alternative, the economic benefits were also necessary to assess as this project, and others, would need to be financially 
feasible for the customer. In the case of this transportation project, as is the case with many others, it is not a direct revenue generating project. Rather, the time savings can be translated in to financial savings by quantifying the average cost of time for the local citizens compounded by the annual number of vehicles passing through this area. For this study, data from the US Government Census quick facts website was used to estimate this financial value, by taking the 2015 median household income of $\$ 34,090$ divided by the standard number of working hours in a year, 2000. (US Census Bureau 2016) AADT data collected by the Morgantown Monongalia Metropolitan Planning Organization (MMMPO) was also used to estimate the annual users of these facilities.

This financial model included the travel time savings along University Avenue in both directions within the shared space, as well as the travel time saved within 300 feet of the nearest upstream intersection in either direction. These intersection were at North High Street south of the shared space, and Stewart Street north of the shared space. This data was collected within the model in addition to the original data collected within the shared space confines. These travel time measurements along a 300' stretch of University Avenue at the furthest extremes of the network within the model helped to visualize the impact the shared space will have on the network as a whole in Morgantown, outside of the shared space boundaries. Queue length and delay values were also calculated at the North High Street and Stewart Street intersections as well to capture the complete impact the shared space would have on neighboring intersections. 


\section{RESULTS AND ANALYSIS}

\subsection{Case Study Traffic Data}

After running each of the iterations of the models created, data was collected as described in the methodology section. In addition to the key points of evidence described in the methodology, additional data was also collected to understand more clearly and definitively the overall effects of shared space. The results were then downloaded into Microsoft Excel where the data could be analyzed. Overall, the evidence of shared space improving traffic congestion metrics and being financially feasible were very positive. The following sections describe in more detail the results and conclusions which were drawn from the data.

\subsubsection{Travel Time Results}

The travel time of all vehicles traveling in both directions between Clark Hall and the Business and Economics Building along University Avenue was measured over the course of the entire 60 minute simulation time frame. Figure 25 graphically represents these results. We can see that the average travel time was lower for all 6 iterations of the shared space simulation compared to the current traffic scenario. On average across the six shared space simulations, the travel time for vehicles decreased by $13 \%$, and by $19 \%$ for simulation number 1 which represents the best estimate of pedestrian behavior. Although the average showed a significant drop across all shared space simulations, the median travel time did not. This value actually increased for half of the shared space simulations and decreased slightly on the other half. The reason behind this phenomenon can be seen in the standard deviation of the travel time observations. We can see that the standard deviation drastically decreased from the current scenario to all six of the shared space simulations. This equates to the risk of a driver not being able to traverse the 
prescribed area within the average time. The variance of the shared space travel time is significantly lower than that of the current scenario, meaning drivers could expect a much more consistent commute within the shared space. The current scenario had multiple outliers which stretched the variance of the observed travel time data to an extreme extent.

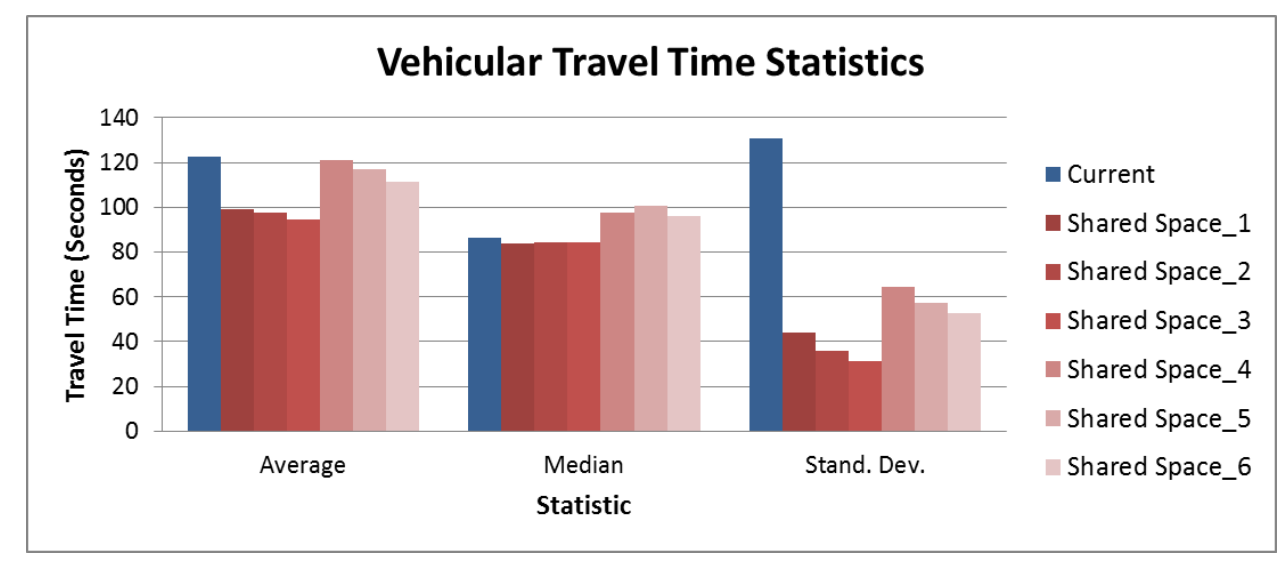

Figure 25: Vehicular Travel Time Statistics

Figure 26 exhibits the presence of extreme outliers, where $7.63 \%$ of all vehicles traveling along University Avenue in the current model incurred a total travel time of more than 375 seconds. Compare this to the shared space simulations which have barely any occurrences across all six models above 200 seconds of travel time. It can also be seen that minimum travel time, or free flow travel time, for the current scenario is lower than the shared space simulations. Remember that this is due to the inherently lower speed limit set as described in the methodology within the shared space models at half of the current speed limit. This short free flow travel time however is counterbalanced by the extreme outliers on the other end of the spectrum. Therefore in the current scenario drivers have the chance of experiencing very little traffic and getting through quickly, but risk being stuck in the queue for a long time if they do hit the inevitable traffic. On the other hand, in the shared space simulation it is almost certain that a driver's travel time would be within a much smaller range. 


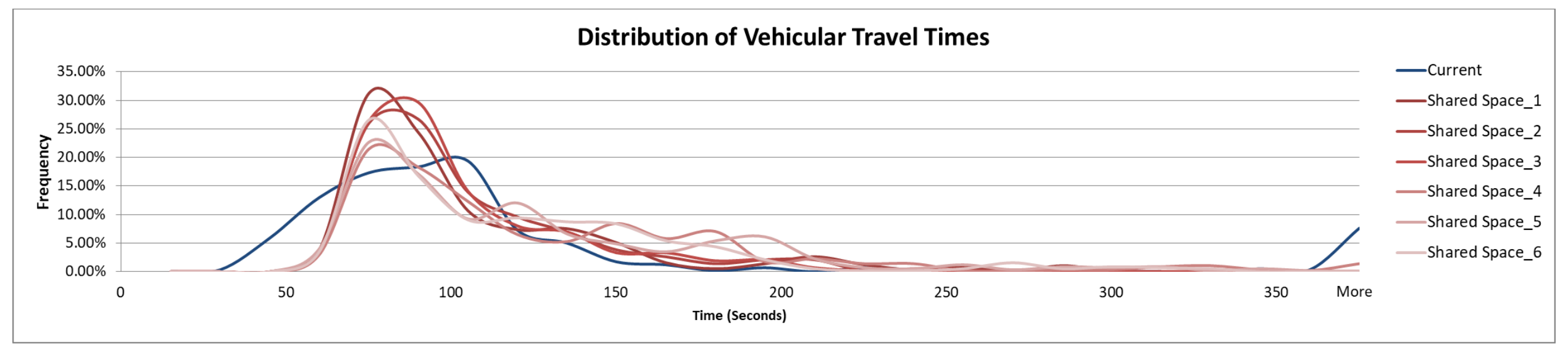

Figure 26: Distribution of Vehicular Travel Times 
To gain a better understanding for the behavior of the shared space and how it reacts to the peak volume of pedestrians, the travel time data was broken down further in to two categories for vehicles traveling through the zone under normal pedestrian conditions and a second group of vehicles traveling through during the 15 minute peak pedestrian volume period. Figure 27 below shows how the shared space performed in terms of travel time for vehicles served during the non-peak pedestrian time period. We can see that these statistics closely match the average travel time statistics over the course of an hour. The non-peak pedestrian period represents 45 minutes of simulated time in these models. The average travel time of the shared space models still exhibited a decrease compared to the current with the exception of model 4 . Model 4 arranged the pedestrian crossing traffic and volumes in a manner which was the most like the current scenario, representing the worst case scenario if the pedestrian crossing behavior remained mostly centralized to one location. The median travel time during the non-peak period was observed to be even closer within this portion of the data as compared to the complete hour of data. Again we can see that this is mostly linked to the behavior of the standard deviation of the current data. Since many of the extreme outliers were present during the peak pedestrian period, they were not included in this portion of the data and therefore reduced the standard deviation by around 20 seconds from 130 to 110 seconds.

Figure 29 shows how the data for the travel times is grouped much closer to the median value than the previous analysis. Still, the current scenario exhibits a shorter free flow travel time counterbalanced by the presence of $4.88 \%$ of the sample being extreme outliers above and beyond 375 seconds. The shared space on the other hand maintains a low median with very few outliers, especially as high as 375 seconds. This brief analysis of the travel times during the nonpeak pedestrian period helps us understand the behavior of the shared space while it is not under 
the stress of a large volume of pedestrians. Even though the speed limit was cut in half within this case study area, we can still see that the shared space performs as well, and in many ways better than the current scenario. We will now see where the shared space clearly outperforms the current scenario, during the peak pedestrian period.

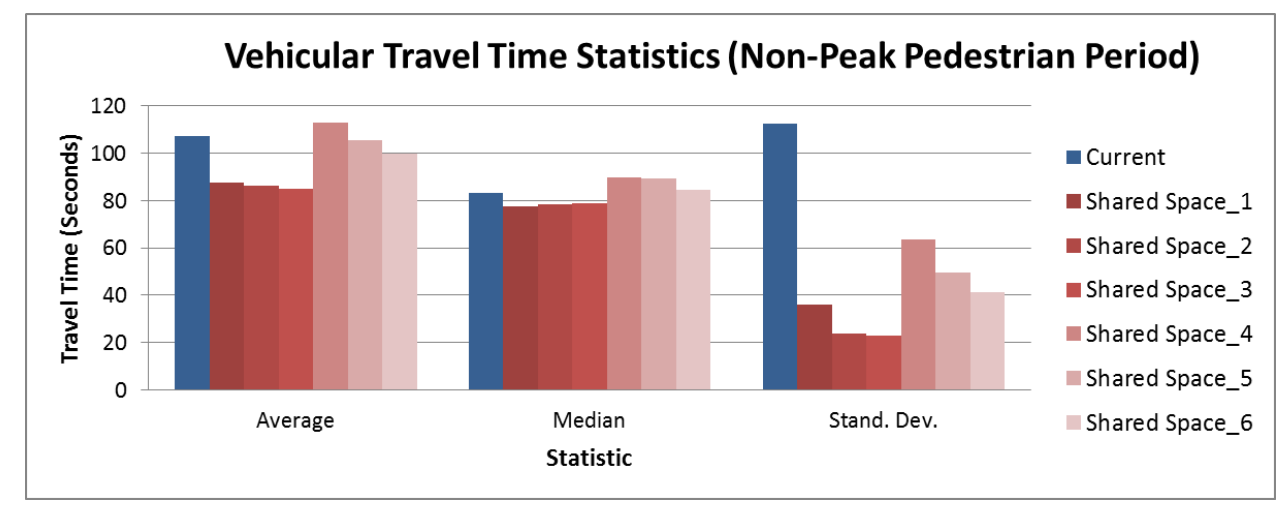

Figure 27: Vehicular Travel Time Statistics During Non-Peak Pedestrian Time Period

The second set of data extracted from the entire set corresponded to travel times of vehicles traversing the study area during the peak pedestrian volume time period. Figure 28 summarizes the statistics of this data and shows the significant difference between the performance of the current design and the shared space models. As we noted earlier, during the non-peak periods the average and median values observed for the shared space models were only slightly lower and equivalent respectively compared to the current model. In the other 15 minutes of the simulation however, during which the peak pedestrian volume is present, we can see a steep decrease across all three statistics for every shared space model.

First looking at the average travel time, we can see that the first shared space model decreased by more than 170 seconds. On average, the shared space models decreased the travel time by 166 seconds, which represented 54\% of the current travel time for vehicles during the 
peak pedestrian period. The median and standard deviation averaged across all six shared space models also showed significant drops of $42 \%$ and $72 \%$ respectively.

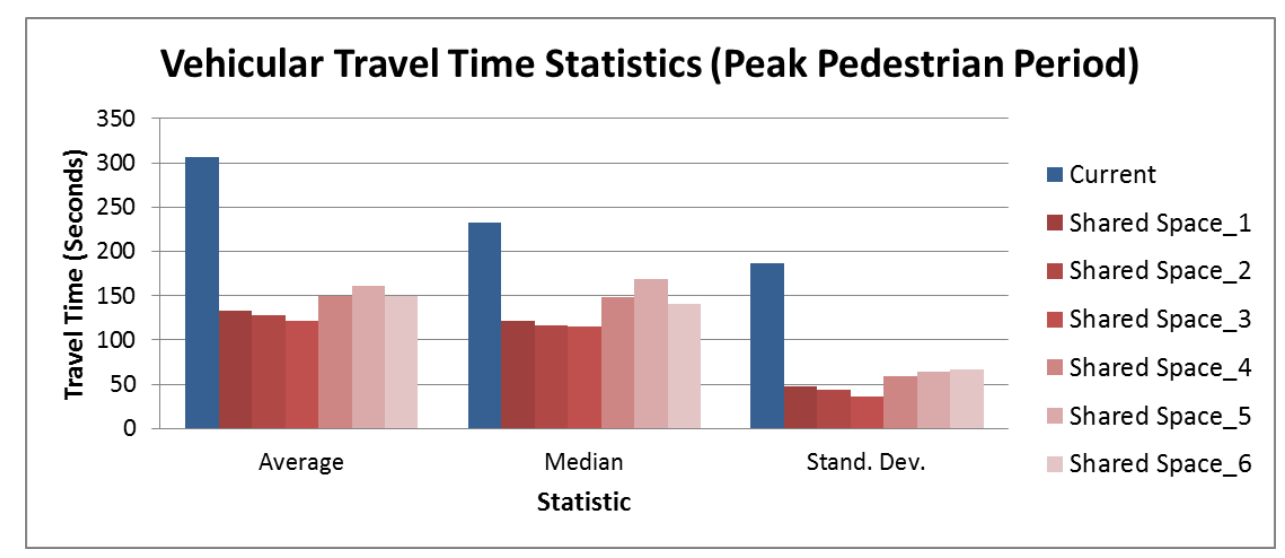

Figure 28: Vehicular Travel Time Statistics During Peak Pedestrian Time Period

As was discussed earlier, the current model travel times show much more inconsistency and variability compared to the shared space models. This point is reiterated in Figure 30 which shows the distribution of travel time occurrences during the peak pedestrian period. We can see here that more than $40 \%$ of drivers observed in this time period experienced a travel time exceeding 375 seconds in the current model. On the other hand, some of the shared space models had no observed travel times above 250 seconds. The models that did, had very few drivers above this range. Although in the previous two data sets it was noted that the free flow travel time was consistently shorter for the current model, in this case during the peak pedestrian period we see the opposite. Each of the shared space models during the peak pedestrian time period were observed having a shorter minimum travel time than the current model while maintaining a small range of observations in comparison to the current model. The current model on the other hand shows a significantly longer minimum travel time with multiple observed travel times up to 250 seconds, and nearly half of the observation in the extreme outliers. 


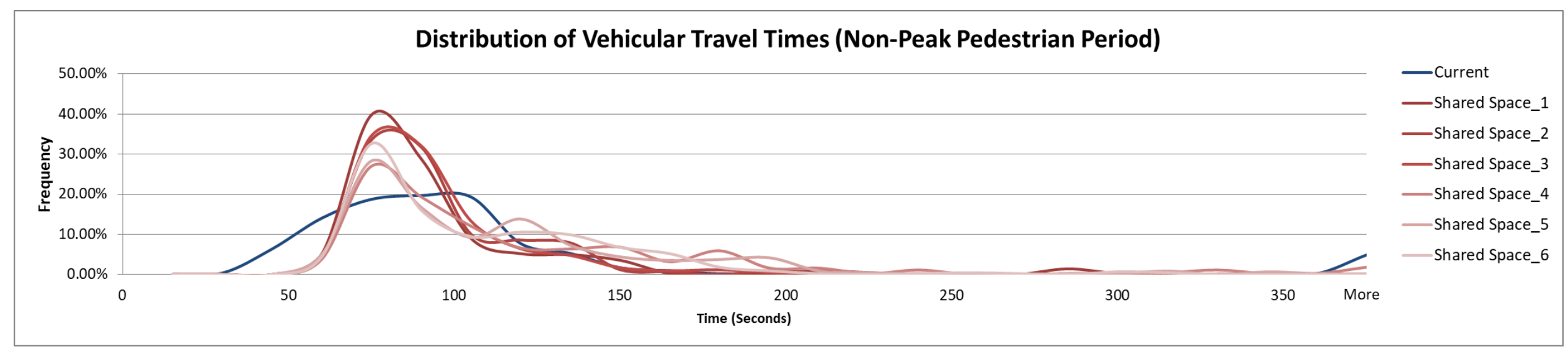

Figure 29: Distribution of Vehicular Travel Times Observed During the Non-Peak Pedestrian Time Period

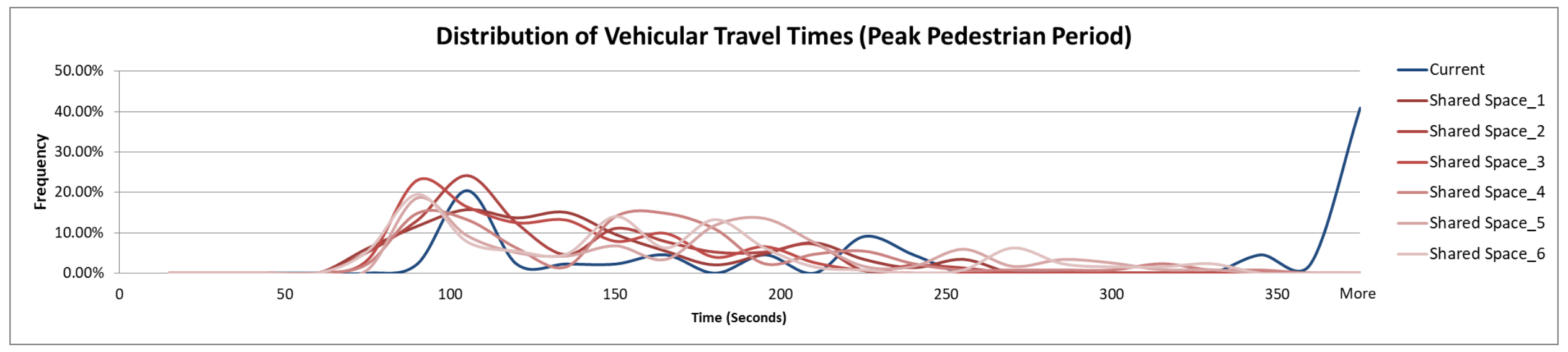

Figure 30: Distribution of Vehicular Travel Times Observed During the Peak Pedestrian Time Period 
These results also made the research team take notice of the number of observed data points, or vehicles, during the peak pedestrian time period of 15 minutes. Since all of the models were identical in terms of vehicular behavior and volumes, the number of observations during the peak time period should allow us to observe the flow of vehicles able to be serviced by the facilities during the peak time period. Figure 31 shows the vast difference in vehicular flow between the current and shared space models. Even though over the course of the entire hour long simulation, approximately the same number of vehicles were served at the case location, the current model could only serve less than a third of the amount of vehicles as the shared space models. This effectively shows the additional amount of vehicles that would need to be served in the non-peak pedestrian time periods by the current model. We will see later the effects that this had not only on the case study location but also surrounding intersections as well. This data provides evidence that the current study location is certainly broken as the vast majority of vehicles are not being served as they arrive.

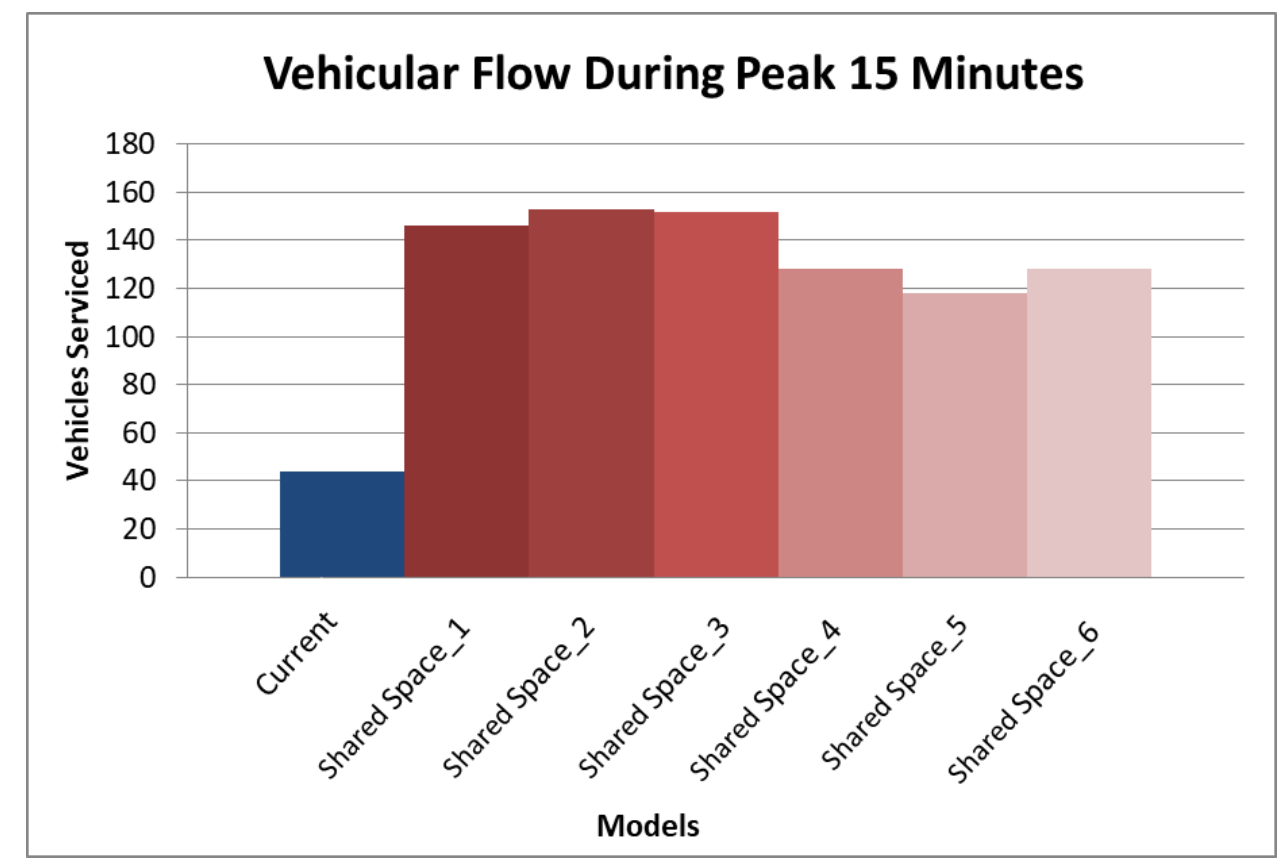

Figure 31: Vehicular Flow during Peak Pedestrian Volume Period of 15 Minutes 
Finally, the travel time data was divided in to the northbound (NB) and southbound (SB) directions to see how the shared space affected travel in either direction. It was found that the southbound direction performed better than overall average and showed decreases in average, median, and standard deviation. The northbound direction shared space observations didn't show much change in comparison to the current observed values though. Figure 32 shows how the statistics of the northbound direction are more consistent with the overall shared space averages discussed earlier. The reason the southbound direction is performing seemingly better is that there are no side streets on the southbound side of the road, whereas the northbound side has two, Prospect Street and College Avenue. These streets not only add additional volume in the northbound direction but also cause delay with exiting and entering vehicles.

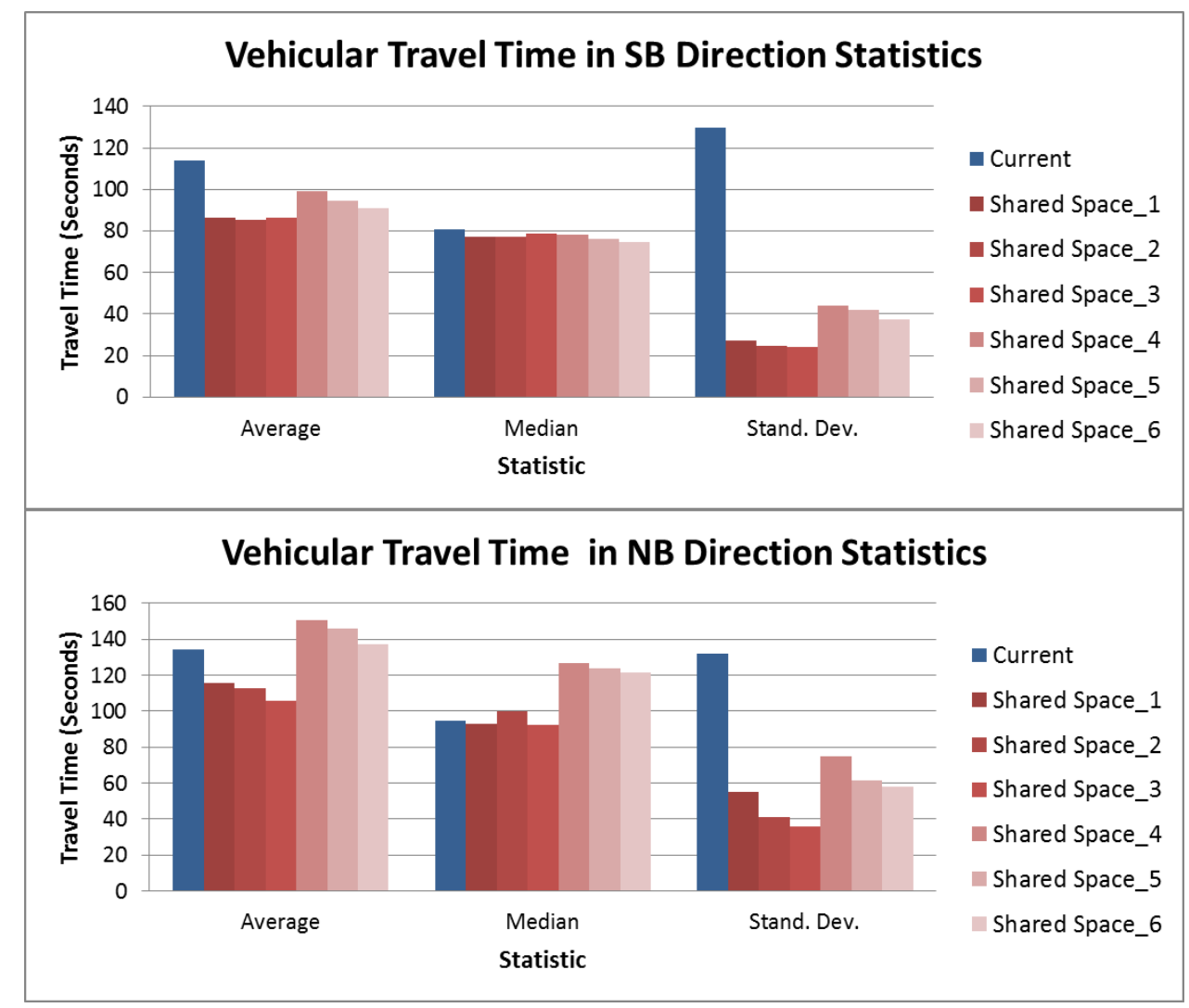

Figure 32: Vehicular Travel Time Statistics split in SB and NB Directions 
In addition to analyzing and comparing the raw travel time data from the seven separate models, a PERT analysis was also performed. The PERT technique, or Program Evaluation and Review Technique, is typically used in project management applications to gain a better understanding for the expected duration of a project or program. This projected duration is calculated using the minimum, mode, and maximum duration expectations in a weighted average format as shown in the equation below.

$$
\text { Mean or Expected Duration }=\frac{\text { minimum }+4 * \text { mode }+ \text { maximum }}{6}
$$

In the case of this shared space simulation analysis, this method was chosen to put in perspective the potential of having an extremely high travel time in the current scenario compared to in the shared space model. The raw travel time data was first rounded up to the nearest increment of 15 seconds. The mode for the current model was then found to be 105 seconds, compared to the shared space models which had modes between 75 and 90 seconds. The maximum of the current model was found to be 750 seconds, or 12.5 minutes. On the other hand the shared space model maximums ranged between 225 seconds and 405 seconds. Finally, the minimum value for the current model was 30 seconds and the shared space models all had minimum values of 60 seconds. Again, this is due to the halving of the speed limit in the shared space models.

This information was then used to calculate the PERT values for each of the seven models. The calculation results indicated that the current scenario would have a mean or expected travel time of 200 seconds. Comparatively, the shared space model PERT expected travel times ranged between 107.5 and 127.5 seconds, with an overall average expected value of 115.4 seconds. That is an 84.6 second drop, or $42.3 \%$ decrease, in expected travel time from the 
current scenario. These significant decreases help us put a better emphasis on the drastically smaller range of expected travel times between alternatives, and the effect that the potentially extremely long travel times have on the current drivers.

Table 2: PERT Expected Travel Times for Each Model in Seconds

\begin{tabular}{|c|c|}
\hline VISSIM Model & PERT Expected Travel Time (seconds) \\
\hline Current & 200 \\
\hline Shared Space_1 & 110 \\
\hline Shared Space_2 & 112.5 \\
\hline Shared Space_3 & 107.5 \\
\hline Shared Space_4 & 127.5 \\
\hline Shared Space_5 & 120 \\
\hline Shared Space_6 & 115 \\
\hline
\end{tabular}

Overall, the travel time of the shared space models slightly improved compared with the current model data, and the PERT expected travel time improved tremendously. Even with the presence of a speed limit half as a high as the current, the shared space was able to consistently service the vehicles in a timely manner. The consistency of the travel times in the shared space observed through the standard deviation should also be noted as is emphasized in the PERT calculation that showed the most significant change among the travel time statistics and will have a direct impact on the driver experience at the case location for travelers. By now reviewing the delay statistics of the shared space models, we'll be able to see how the shared space design was able to maintain a consistently low travel time with a much lower speed limit. 


\subsubsection{Delay Results}

The delay data results were also analyzed in order to ascertain the changes in vehicular travel behavior, and how often they were unable to travel uninhibited through the case study location in the various models. The delay measurement is very important as this is what the user will perceive as traffic experienced within the area. The delay results were even more positive than the travel time results in favor of accepting shared space as a solution at the case location. Whereas in the travel time statistics exhibited a slight decrease in the average and median, the delay statistics decreased more dramatically.

Figure 33 Summarizes the statistics for the vehicular delay average over the entire 60 simulation period. We can see that the average delay decreased by nearly $50 \%$ for the average shared space model, and $56 \%$ for the primary shared space model. The resulting average delay for all six shared space models is 43.57 seconds across the entire study area. It should also be noted that the median and standard deviation also decreased significantly, and more so than the travel time statistics did. To reiterate, this significantly lower standard deviation of observed delay measurements for vehicles corresponds to a much more reliable and predictable experience for drivers. Unlike in the current case in which drivers could experience barely any delay up to and beyond a six minute delay, in the shared space models we are showing over the observed hundreds of vehicles in the course of an hour, the range of delay values is almost completely confined by zero and two and a half minutes of delay. For a driver planning their commute, this significant drop in risk of getting stuck in traffic for an extended period of time would be a huge relief. 


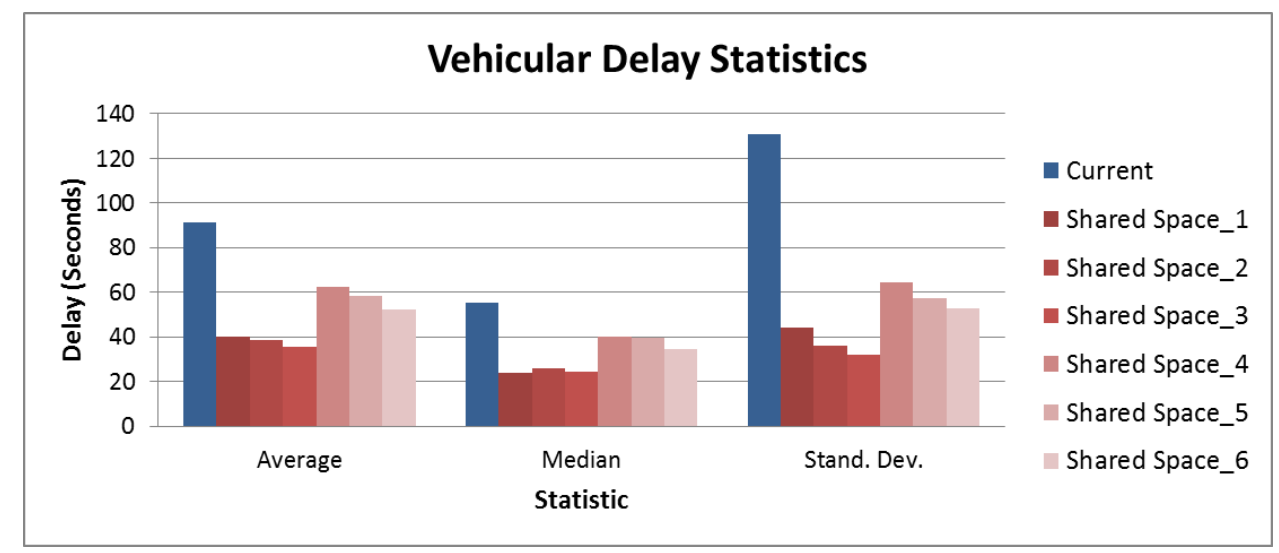

Figure 33: Vehicular Delay Statistics

By evaluating the distribution of observations in Figure 34 for the vehicular delay we can see how all of the observations from the six shared space models are highly congregated towards the lowest bin at 15 seconds. The remaining shared space observations tail off quickly with the rare occurrence of an observation above 150 seconds. In the current model on the other hand, the highest percentage of observations doesn't fall until the 60 and 75 second bins with approximately $19 \%$ of the observations in each. The observations for the current model also tail off quickly up to the 150 second point; however there is again the presence of a large group of extreme outliers. Just like for the travel time statistics, $7.63 \%$ of the observed vehicles experienced a travel delay in excess of 375 seconds. To put that in perspective, this means that in the current scenario, a driver could expect to experience a delay of between 0 and 150 seconds $90 \%$ of the time, but will inevitably be delayed by more than six minutes the remaining nearly 10 percent of the time. We will see in the following results that the majority of these observations were during the pedestrian peak period.

The overall data was again split between the peak and non-peak pedestrian periods. Figure 35 shows the three statistics for the split data, both from the peak and non-peak periods. Across both periods we can see a consistent drop in all three statistics. The larger decrease 
however can be seen in the peak pedestrian period timeframe statistics. The ability of the shared space to maintain a relatively low level of delay even during the peak pedestrian period is a positive sign that it will be able to handle the influx of crossing pedestrians in a much more effective manner than the current design. As was discussed earlier, the idea that even with a high volume of pedestrians the vehicles in a shared space will be able to maintain smooth and steady progress through the case location is supported through this data. The decrease in average, median, and standard deviation in the peak period was significantly larger than in the non-peak period due to the extremely poor performance of the current design during the peak period.

Although the decrease wasn't as drastic during the non-peak period, the fact that the decrease was still relatively large and the point to which the delay in the shared space was reduced to is significant. Among the shared space models, the average delay and median delay were reduced to between 20 and 60 seconds, and 20 and 30 seconds respectively. Over the course of the entire shared space, a delay value this low would be approaching a negligible delay for drivers. These low delay measures serve as evidence to positively support that shared space has the capacity and ability to serve both the pedestrian needs and vehicular volume demands even during peak periods and will make a measurable and perceivable difference for the users. 


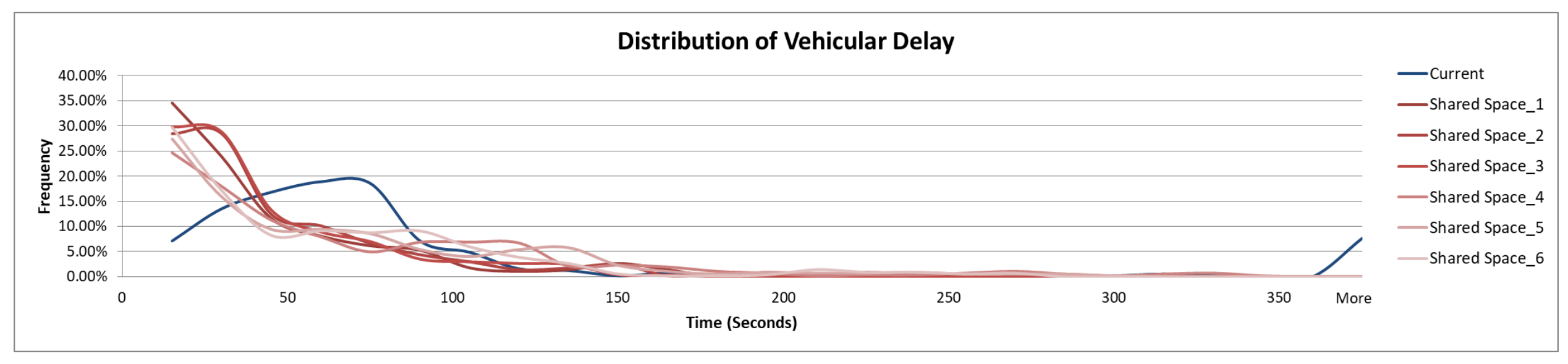

Figure 34: Distribution of Vehicular Delay

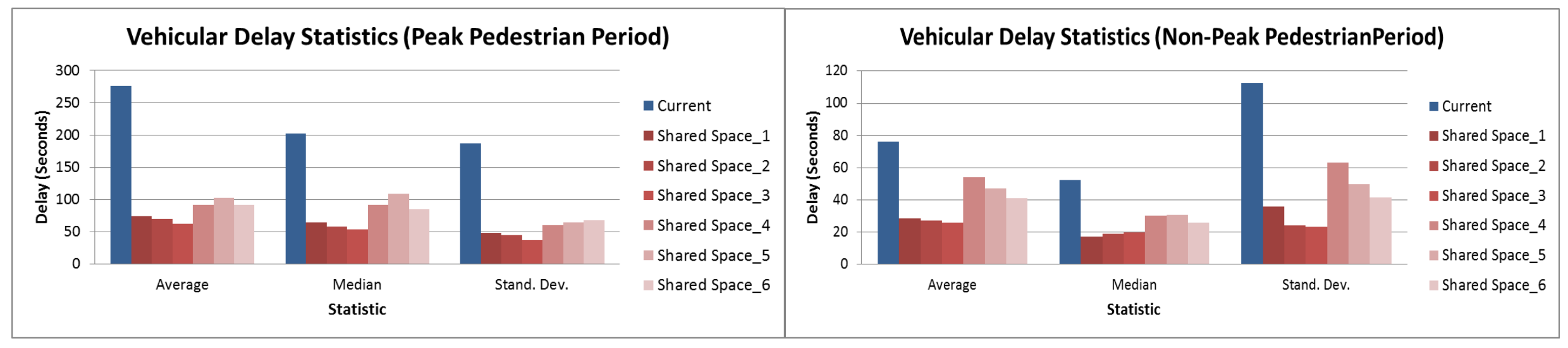

Figure 35: Vehicular Delay Statistics Split Between Peak and Non-Peak Pedestrian Time Periods 
Looking back at the distribution graph for vehicular delay in Figure 34, it was noted that most of the extreme outliers which incurred more than six minutes of delay were mostly confined to the pedestrian peak volume period. Comparing the distribution of observations in Figure 36 and 37 we can see how this is true. First looking at Figure 37, which show the nonpeak time period vehicular delay, it can be observed that the amount of extreme outliers above six minutes has decreased slightly, while the majority of the observations remain at 100 seconds or less for the current scenario. Meanwhile, the shared space models also exhibited nearly all vehicles having less than $100-150$ seconds of delay, plus the proportion of vehicles experiences little to no delay, in the lowest bin, was much higher in comparison to the current model. Additionally, the shared space model data didn't include any extremely high outliers as the current model did.

Then if we compare these results to the ones displayed in Figure 37, we can see how even though the shared space models were able to retain a low level of delay with all vehicles experiencing less than 300 seconds of delay and the majority less than 150 seconds, the current design had a much more difficult time coping with the traffic under duress of the peak pedestrian volumes. Approximately half of the vehicles in the current model were observed experiencing between 50 and 200 seconds of delay, while the remaining vehicles experienced significantly higher delays. Of these higher delays, more than $40 \%$ of the overall vehicles experienced a delay in excess of six minutes just within the confines of the study location. These results coincide with the findings of the travel time study, indicating that the shared space design can handle more effectively the influx of pedestrians in the system. This ample ability to handle the vast number of pedestrians during the peak time then also reduces the amount of traffic delay which would run over in to the non-peak pedestrian time as the current scenario did. 


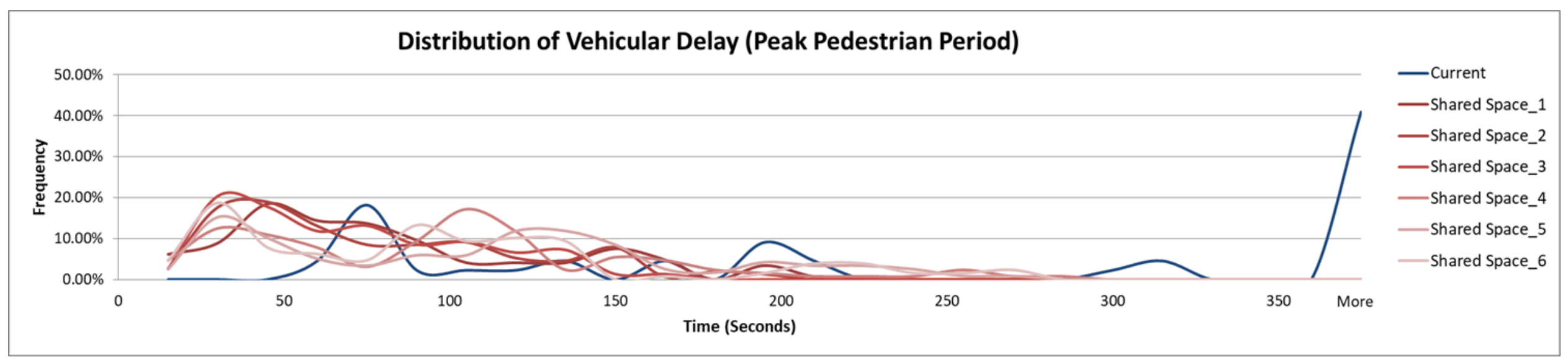

Figure 36: Distribution of Vehicular Delay during the Peak Pedestrian Volume Period

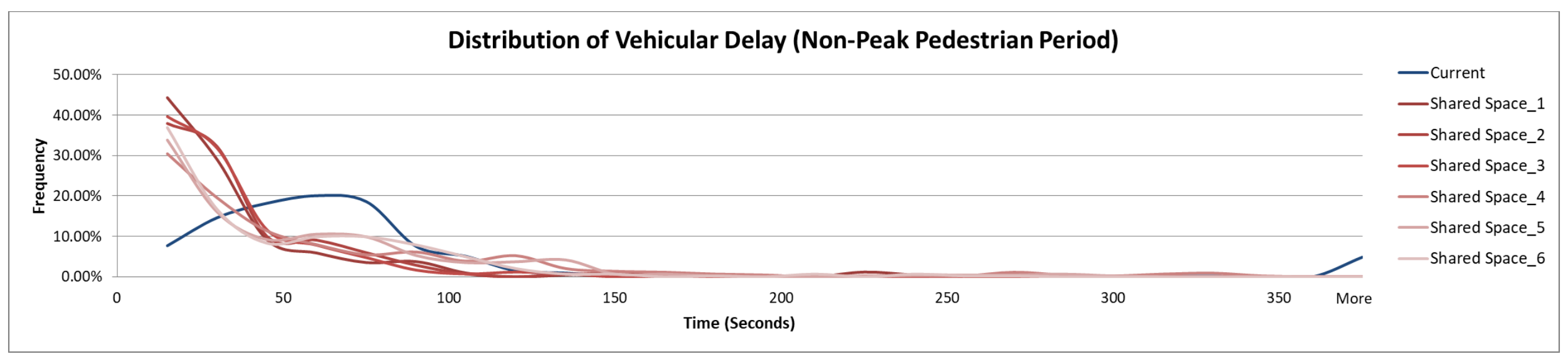

Figure 37: Distribution of Vehicular Delay during the Non-Peak Pedestrian Volume Period 
Finally, the delay data was split between the north and southbound directions to identify and differences between the two. As shown in Figure 38, the overall conclusion made for the average of both directions is still consistent when split between North and Southbound. We can notice however, that as was similarly observed in the travel time statistics, the southbound direction within the shared space model performed significantly better than the Northbound direction when isolated. As explained earlier, this is due to the higher vehicle volumes in this direction, and the additional cross streets which interrupt steady flow on that side of the street.

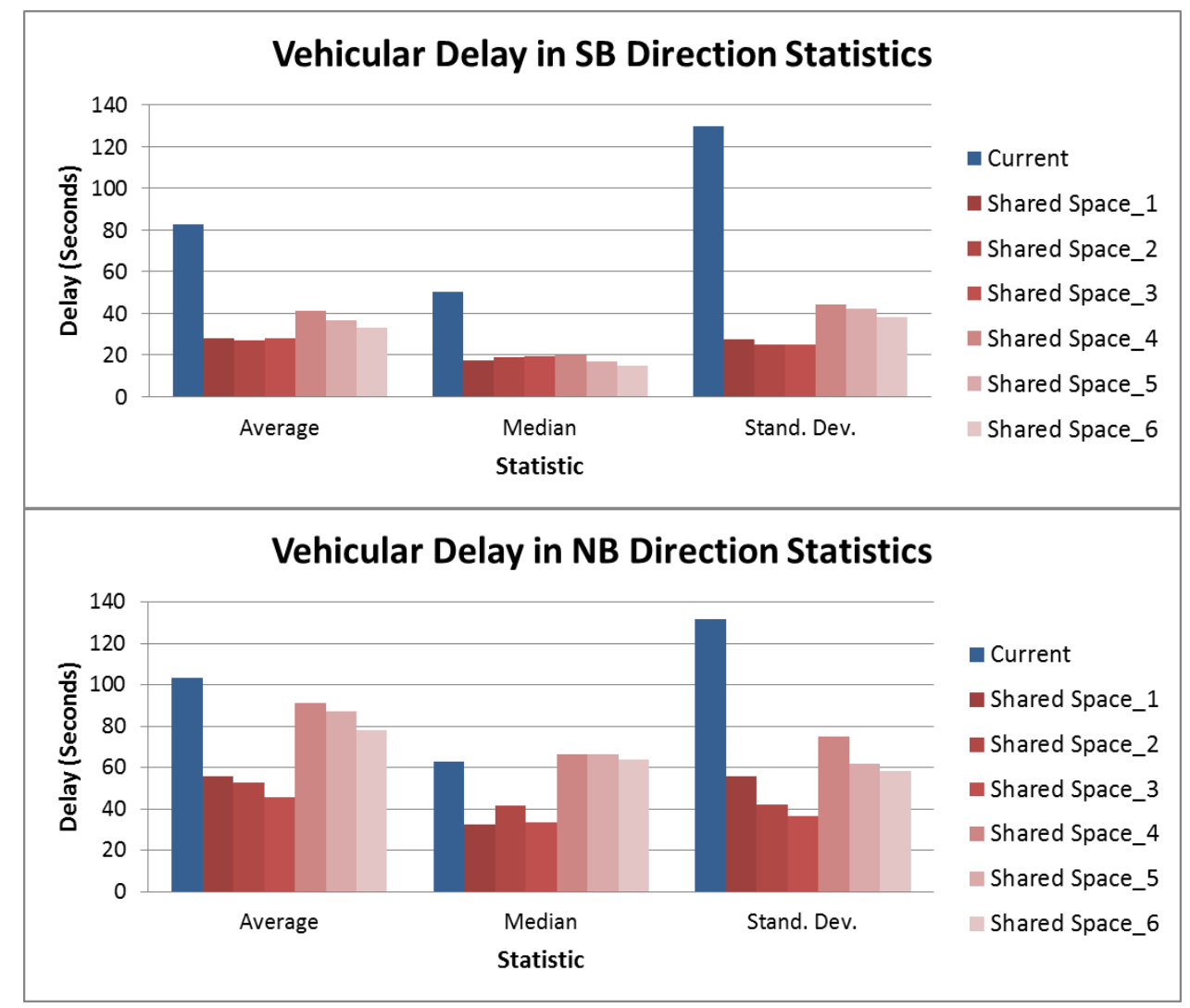

Figure 38: Vehicular Delay Statistics Split Between Northbound (NB) and Southbound (SB) Directions

None the less, the Northbound direction still performed consistently better than the current model, especially when looking at the first shared space iteration which was the primary model. We can point out that shared space models four through six did not perform as well as we 
had hoped in the Northbound direction. The reason for this is quite simple and understandable though. When making the multiple iterations of the shared space model to have resilient results, models four through six were designed to have more compact flows of pedestrians crossing University Avenue, which would be most similar to the current behavior of pedestrians. These iterations also emphasized the peak volume of pedestrians during the 15 minute period more aggressively than the other models. All of these strategies for creating additional shared space iterations were designed not only to make the results and the models more resilient, but to more importantly show the most conservative results possible. Some of the future behaviors of pedestrians is unknown, and we want to ensure that in all cases the shared space design will perform better. These strategies allow us to do so.

The PERT analysis was again performed on the delay data observed in the models. In a similar fashion to the travel time data, the delay data was also rounded up to the nearest 15 seconds before calculating the mode. For the current model the delay mode was 60 seconds, with the shared space model modes ranging between 15 and 30 seconds. Since 15 seconds is the lowest possible number, we can already see how little delay there is min the shared space design. The maximum delay for the current model was an incredible 705 seconds, or 11.75 minutes. Meanwhile, the shared space delay maximums ranged in a much more reasonable range from 165 to 345 seconds. Finally the minimum delay value for the current model was 0 seconds, and the shared space models had minimum values of either 0 or 15 seconds. With these values the PERT mean or expected delay was calculated. The current model expected delay was found to be 157.5 seconds. The six shared space models returned PERT expected delay values ranging between 37.5 and 67.5 seconds, with an average of 54.6 seconds. This represents a 102.9 second, 
or 65.34\%, drop in delay between the current and shared space designs. Again, this PERT calculation helps show the significant advantage of utilizing shared space in this scenario.

Table 3: PERT Expected Delay Calculated Values for all Seven Models

\begin{tabular}{|c|c|}
\hline VISSIM Model & PERT Expected Delay (seconds) \\
\hline Current & 157.5 \\
\hline Shared Space_1 & 50 \\
\hline Shared Space_2 & 52.5 \\
\hline Shared Space_3 & 37.5 \\
\hline Shared Space_4 & 67.5 \\
\hline Shared Space_5 & 62.5 \\
\hline Shared Space_6 & 57.5 \\
\hline
\end{tabular}

Overall the results from the delay data were very positive in favor of utilizing shared space. Across each of the models the delay was decreased significantly. The PERT analysis helped show the drastic drop in the expected delay, and by analyzing the peak and non-peak demand period, we could see the improved handling of pedestrian crossing influxes in the shared space model. These low delay volumes will also relate to shorter queuing lengths, and less impact on the surrounding areas of the transportation network, as we will see data of in the following sections.

\subsubsection{Queue Length Results}

In addition to the travel time and delay data, data on the queue length beginning at the current Grumbein's island and extending in either direction were collected. This data should mirror our findings for the delay data as the vehicles in a queue are also considered to be in a delay. Figure 39 shows the queue length looking north from Grumbein's island, and represents the drivers heading southbound. We can clearly see in this graphical representation of the queue lengths that the primary shared space model queues remain short throughout the simulation 60 
minute period, while the current queue length rapidly increases with the addition of pedestrians and takes nearly the entire hour to dissipate the queue. The fourth through sixth shared space simulations also exhibited a quick jump in maximum queue length, but unlike the current model queue, were able to quickly dissipate with the return of pedestrian volumes to their normal levels.

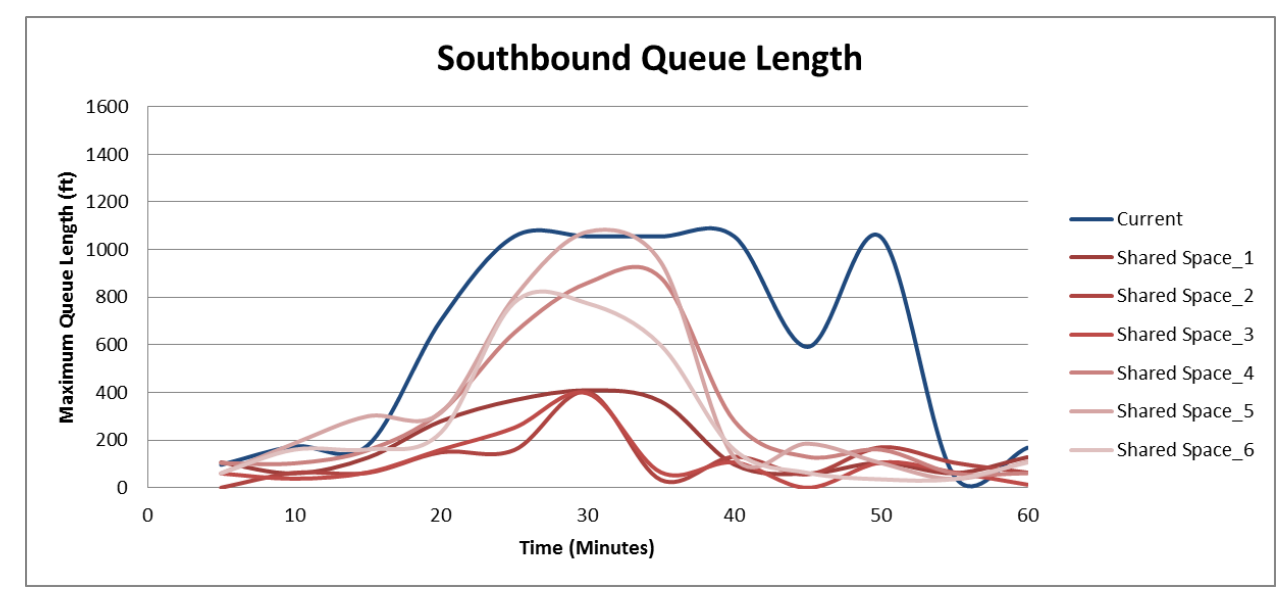

Figure 39: Maximum Queue Length in 5 Minute Intervals Measured for Vehicles Traveling in the Southbound Direction

Likewise, the northbound direction vehicle queues can be seen in Figure 40. As we discussed previously, the delay and queue lengths in the northbound direction were larger in comparison than the southbound direction due to the larger vehicular volumes and entering and exiting traffic on the two cross streets. The behavior of the queue lengths for the primary shared space models though is still consistent in nature as before, as the queues do not increase as rapidly as the current scenario, remain at a shorter level, and also dissipate more quickly. The northbound direction current queue again jumps to a high maximum length quickly and remains high throughout the majority of the remainder of the simulated 60 minutes. We do notice however that the fourth through sixth shared space simulations also reached the same maximum queue length as the current scenario. Again, this is most likely due to the more conservative 
pedestrian distribution and volumes used in this model, however none the less, those simulations still performed as well if not better than the current scenario in terms of queue length.

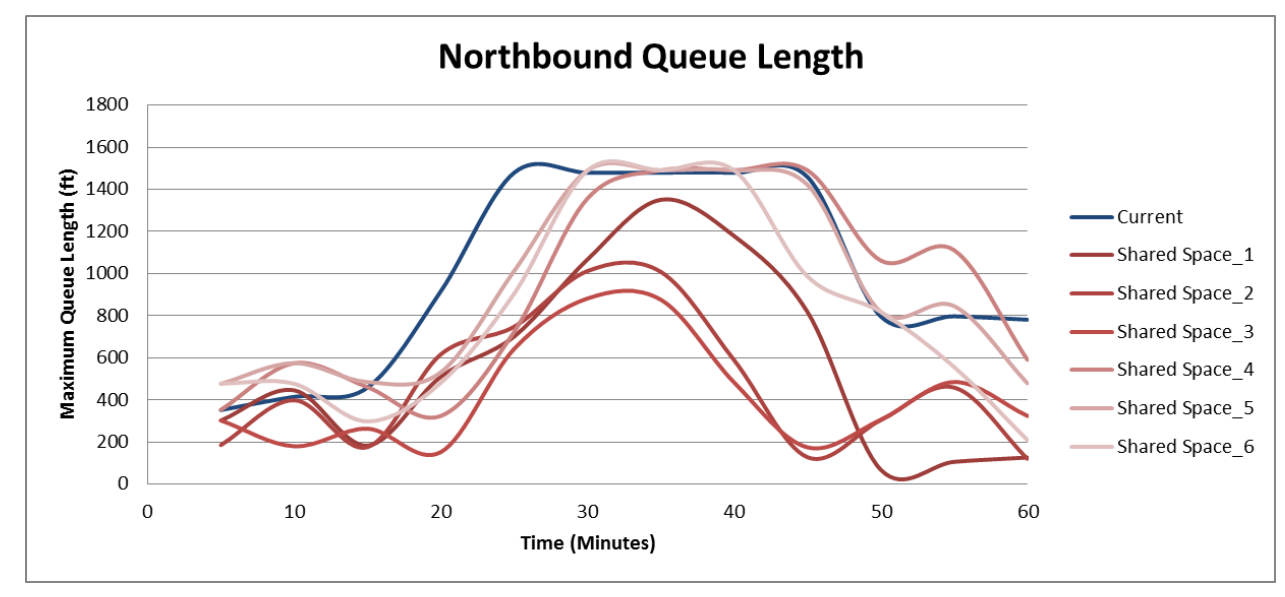

Figure 40: Maximum Queue Length in 5 Minute Intervals Measured for Vehicles Traveling in the Northbound Direction

Note that since only the maximum queue length value for each of the five minute periods was collected, the exact behavior of the queues can't be ascertained. For example, if one queue is 500 ' long for the entire five minutes, and another queue is only 500' long for a single moment within that 5 minute time span before dropping back down to a much lower level, they will both show up in these results as having the same 500' maximum queue length during that 5 minute time frame.

\subsection{Network and Economic Analysis}

Beyond the analysis of the traffic statistics within the shared space design, it was also pertinent to examine the effects the implementation of shared space in this case study would have on the surrounding traffic and the network as a whole. To accomplish these observations, additional data collection points were established within the simulation, as described in the methodology, which would collect travel time and queue length data within 300 ' of the next intersection along University Avenue at Stewart Street and North High Street. These findings can 
then not only be used to gather insights about the reach of the effects of the shared space implementation but also be used to formulate a more all-encompassing economic analysis.

The vehicular travel time and delay for vehicles traveling in the southbound direction were measured beginning at Stewart Street and continued for 300' as they approached the shared space. The results can be seen in Figure 41 below. It can be ascertained that the shared space effectively eliminated all of the impact on the traffic upstream on University Avenue north of the case location. We can see that the average and median delay were brought to a minimal amount on only two of the shared space models, and zero on the remaining four, allowing vehicles to travel at the free flow speed. The standard deviation continued to be low if not zero for the delay and travel time as well giving the drivers a very consistent experience.

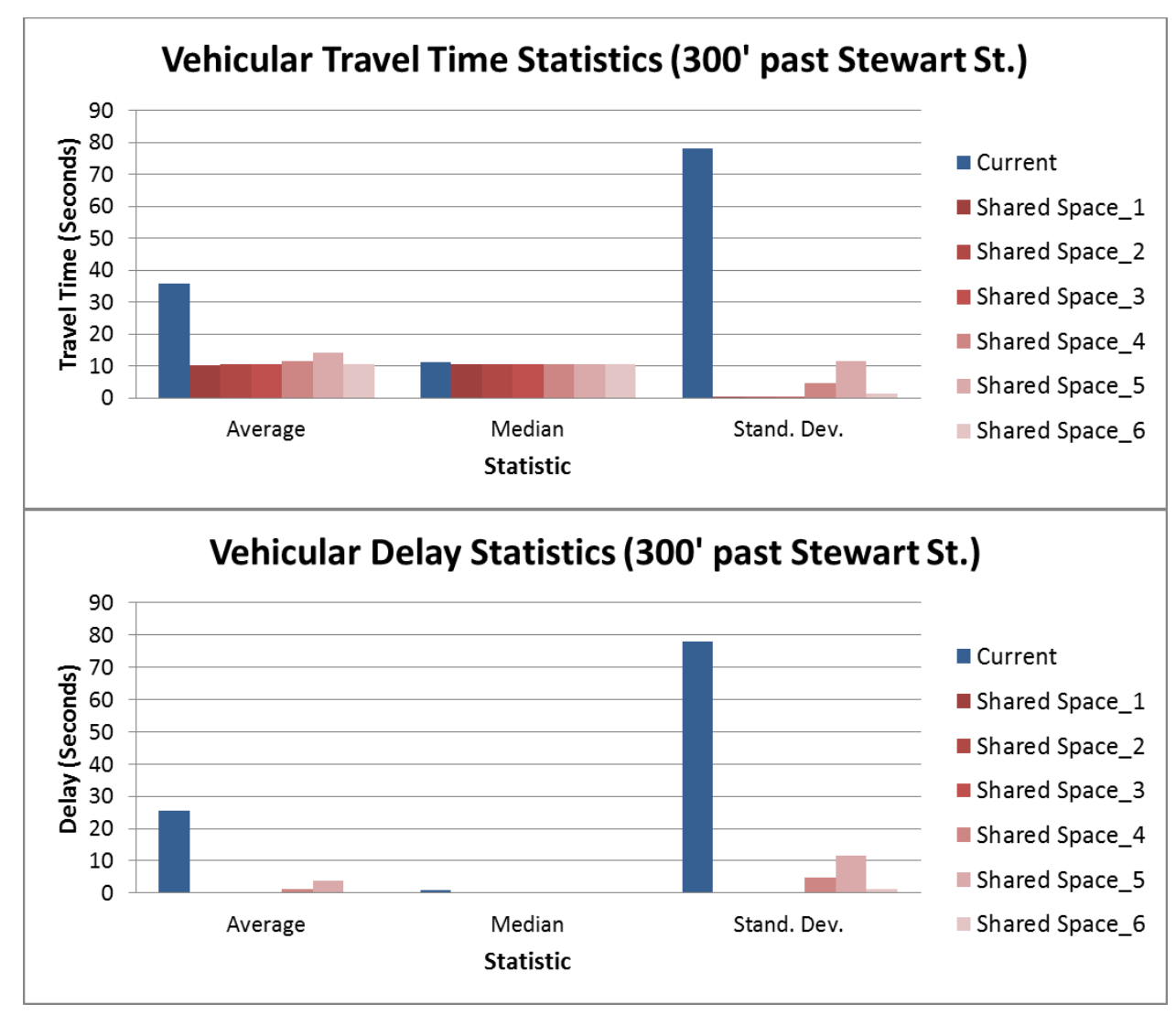

Figure 41: Vehicular Travel Time and Delay Statistics for Vehicles Traveling in the Southbound Direction from Stewart Street to 300 feet Past the Intersection 
In the northbound direction, vehicles were observed within $300^{\prime}$ of passing through the intersection on North High Street. Figure 42 shows the statistical results for those measurements. Again, the impact to the vehicles at this neighboring intersection was reduced by the shared space. We can see that this reduction in impact did not have the same intensity as the southbound direction but still exhibited a positive trend. The delay average and standard deviation both decreased on average by a little less than half for the shared space models and corresponded to an equivalent reduction in travel time average and standard deviation as well. Again, this less severe decrease can be explained by the additional vehicles and cross streets in this direction, and the less amount of distance present between the shared space and this intersection at North High Street compared to between the shared space and Stewart Street.

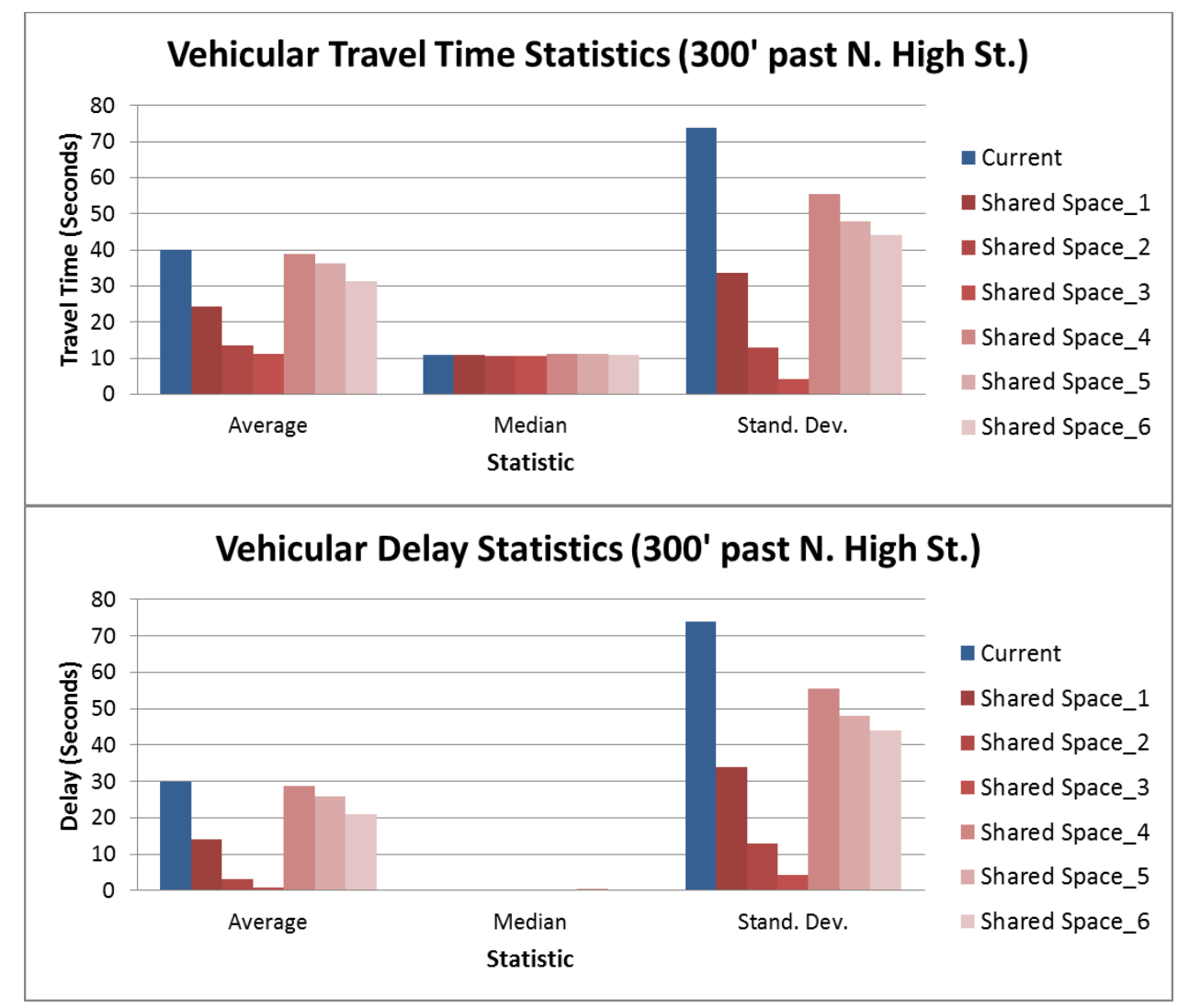

Figure 42: Vehicular Travel Time and Delay Statistics for Vehicles Traveling in the Northbound Direction from North High Street to 300 feet Past the Intersection 
To finalize this network impact portion of the study, the queue length at each intersection was measured beginning 300' away from the intersection and looking in the opposite direction of traffic towards the North High or Stewart Street intersection they just departed from. These results were also very positive as the queue in the southbound direction never formed for the primary shared space model and only briefly formed in the northbound direction for a short amount of time and of less intensity compared to the current model. See Figure 43 below for the results.

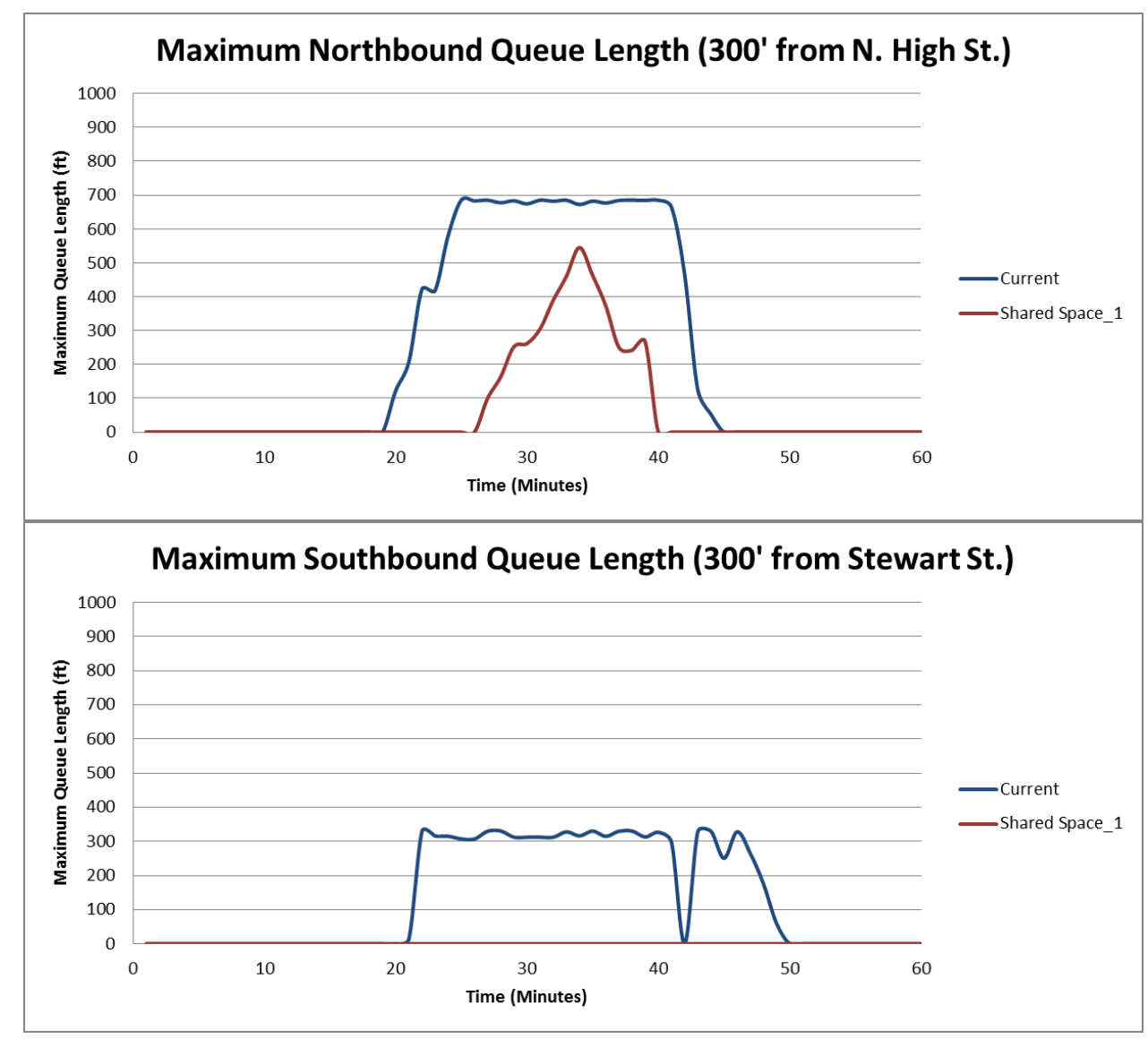

Figure 43: Queue Length 300' Away from Neighboring Intersections Along University Avenue

\subsubsection{Net Present Value and Payback Period}

Although the shared space has proved its ability to improve the case location in terms of traffic aspects, it is also important to check the economic and financial feasibility of this project. 
It has yet to be determined which public entity would hypothetically fund, operate and maintain, and oversee this project whether it is the state, city, or university officials. With the current economic and budgetary constraints within the State of West Virginia it was even more important to show the tangible economic benefits gained from this project.

The economic analysis was conducted based on the principle that citizens, students, and travelers within Morgantown will save time travelling each day due to the introduction of a shared space environment. This will effectively be saving them money as each person's time is worth money in terms of an opportunity cost. Travel time can then be traded for more time at work earning money, or more time at home performing leisure activities which bring enjoyment to the individual. Information gathered from the US Census Bureau indicated that the median annual household income in Morgantown between 2001 and 2015 was $\$ 34,090$, and the per capita annual income during that same time period was $\$ 22,101$, both in 2015 dollars. (US Census Bureau) Assuming that individuals will work approximately 2000 hours per year to earn this salary, the money value of time in Morgantown could then be estimated to be between \$11.05 and \$17.05 per hour in 2015 dollars. Between 2015 and 2017 inflation in the US dollar was approximately 3\%, meaning the range for the opportunity cost in Morgantown in 2017 would be approximately $\$ 11.38$ to $\$ 17.56$. For this study, we chose to use a value of $\$ 15$ for the opportunity cost in Morgantown. This means that for every hour saved traveling, the individual will effectively be keeping or gaining an additional $\$ 15.00$. This modest amount then compounded over an entire year, for all travelers in Morgantown, results in significant financial savings each year.

Traffic AADT data from the most recent Morgantown Monongalia Metropolitan Planning Organization traffic study was also used to estimate the daily usage in terms of vehicles 
at the case study location, Stewart St., and North High St. The value at Grumbein's island was observed to be 18,232, and only half, uni-directional, AADT values of 9,789 and 5,188 vehicles per day values were utilized for Stewart St. and North High St. respectively. Two sets of travel time saved values were used to evaluate this project. First, the average travel time reduction between the current and shared space_1 (primary) model was used. The second evaluation used the average travel time reduction between the current and average of all six shared space models. With the seconds saved per vehicle calculated from the model results, and the AADT values from the MMMPO, the total time saved per day could then be calculated. Instead of extrapolating these savings over all 365 days in a year, only 170 days were used. This value was based on the number of weekdays in a year when most WVU students are present. Since the influx of pedestrians were the cause of traffic delay, when there are no students present the shared space will not have the same travel time reductions. Again, this was another way to make a conservative estimate of this project's financial feasibility.

Other ways in which the conservative approach was maintained in the evaluation of shared space included choosing which benefits to quantify and include in the model. The monetary value of travel time saved per year within the shared space and at the neighboring intersections was the only benefit which was quantified in the financial model. Other potential benefits would include aesthetic appeal, increased student enrollment, reduced environmental impact through less idling emissions, reduced vehicle impact through improved pavement, reduced travel time throughout entire Morgantown network, and other subjective benefits which may stem from the introduction of shared space.

Table 4 below shows the calculation of the total money saved per year, as well as the payback period for both the primary shared space model, as well as the average of all six shared 
space models. We can see that using the travel time reduction values from the primary shared space model alone result in a nearly $\$ 535,000$ cost savings per year for the users of this corridor. When using the average values for all six shared space models the savings were valued at just under $\$ 425,000$ in the first year. Since the estimated cost of design and construction for this project was estimated to be $\$ 4,000,000$, the payback period would be around 7.5 and 9.5 years respectively for the two scenarios presented. For a major infrastructure project, this is a very attractive payback period.

Table 4: Total Money Saved per Year and Payback Period Financial Calculation for Primary Shared Space Model and Average of all Six Shared Space Models

\begin{tabular}{|c|c|c|c|c|}
\hline $\begin{array}{l}3 \\
0 \\
\propto 4\end{array}$ & Item & $\begin{array}{c}\text { Primary Shared } \\
\text { Space Model }\end{array}$ & $\begin{array}{l}\text { Shared Space } \\
\text { Model Average }\end{array}$ & Formula \\
\hline 1 & Seconds Saved per Vehicle & 25.41 & 24.54 & $=$ University Avenue and Stewart Street (300') \\
\hline 2 & Total Vehicles per Day & 9,789 & 9,789 & $=50 \% 2016$ AADT from MMMPO \\
\hline 3 & Total Seconds Saved per Day & 248,726 & 240,210 & $=1 \times 2$ \\
\hline 4 & Seconds Saved per Vehicle & 15.80 & 14.13 & $=$ Willey Street and High Street $\left(300^{\prime}\right)$ \\
\hline 5 & Total Vehicles per Day & 5,188 & 5,188 & $=50 \% 2016$ AADT from MMMPO \\
\hline 6 & Total Seconds Saved per Day & 81,963 & 73,299 & $=4 \times 5$ \\
\hline 7 & Seconds Saved per Vehicle & 23.24 & 15.67 & $=$ Current - shared space primary travel time \\
\hline 8 & Total Vehicles per Day & 18,232 & 18,232 & $=2016$ AADT from MMMPO \\
\hline 9 & Total Seconds Saved per Day & 423,712 & 285,695 & $=7 \times 8$ \\
\hline 10 & Net Total Seconds Saved per Day & 754,400 & 599,205 & $=3+6+9$ \\
\hline 11 & Total Hours Saved per Day & 209.56 & 166.45 & $=\mathbf{1 0} / 3600$ seconds $/$ hour \\
\hline 12 & Weekdays with student present & 170 & 170 & $=5$ days $/$ week $\times 2$ semesters $\times 17$ weeks \\
\hline 13 & Total Hours Saved per Year & 35,624 & 28,296 & $=11 \times 12$ \\
\hline 14 & Money Value of Time in Morgantown & 15.00 & 15.00 & $=$ Opportunity Cost in Morgantown \\
\hline 15 & Total Money Saved per Year & 534,367 & 424,437 & $=13 \times 14$ \\
\hline 16 & Cost of Entire Project & $4,000,000$ & $4,000,000$ & $=$ Provided by Stantec \\
\hline 17 & Payback Period (Years) & 7.49 & 9.42 & $=16 / 15$ \\
\hline
\end{tabular}

It is also important to assess the net present value (NPV) and internal rate of return (IRR) for this project as it gives a more accurate analysis of the financial feasibility of projects by including the time value of money. For the NPV and IRR analysis, a 15 year planning horizon was used with a $2 \%$ per year inflation which increased the qualitative benefit each year. Since the funding source for this project is yet to be determined, multiple minimum attractive rate of 
return (MARR) values were used, ranging between 5\% and 11\%. For the primary model, all of the MARR values used between $5 \%$ and $11 \%$ resulted in a net positive present value for the project as a whole, therefore showing that this project would be attractive to an institution with a MARR in this range. The calculation results can be seen below in Table 5 for the primary shared space model. We can see that over the range of $5 \%$ to $11 \%$, the NPV increased from $\$ 267,260$ to $\$ 2,280,844$, corresponding to a benefit cost ratio of between 1.07 and 1.57.

Table 5: NPV and IRR Analysis of Shared Space Primary Model

\begin{tabular}{|c|c|c|c|c|c|c|c|}
\hline MARR & & $5 \%$ & & $7 \%$ & & $9 \%$ & $11 \%$ \\
\hline Year & & Value & & Value & & Value & Value \\
\hline 0 & $\$$ & $(4,000,000)$ & $\$$ & $(4,000,000)$ & $\$$ & $(4,000,000)$ & $\$(4,000,000)$ \\
\hline 1 & $\$$ & 534,367 & $\$$ & 534,367 & $\$$ & 534,367 & $\$ \quad 534,367$ \\
\hline 2 & $\$$ & 545,054 & $\$$ & 545,054 & $\$$ & 545,054 & 545,054 \\
\hline 3 & $\$$ & 555,955 & $\$$ & 555,955 & $\$$ & 555,955 & $\$ \quad 555,955$ \\
\hline 4 & $\$$ & 567,074 & $\$$ & 567,074 & $\$$ & 567,074 & $\$ \quad 567,074$ \\
\hline 5 & $\$$ & 578,416 & $\$$ & 578,416 & $\$$ & 578,416 & $\$ \quad 578,416$ \\
\hline 6 & $\$$ & 589,984 & $\$$ & 589,984 & $\$$ & 589,984 & $\$ \quad 589,984$ \\
\hline 7 & $\$$ & 601,784 & $\$$ & 601,784 & $\$$ & 601,784 & $\$ \quad 601,784$ \\
\hline 8 & $\$$ & 613,819 & $\$$ & 613,819 & $\$$ & 613,819 & $\$ \quad 613,819$ \\
\hline 9 & $\$$ & 626,096 & $\$$ & 626,096 & $\$$ & 626,096 & $\$ \quad 626,096$ \\
\hline 10 & $\$$ & 638,618 & $\$$ & 638,618 & $\$$ & 638,618 & $\$ \quad 638,618$ \\
\hline 11 & $\$$ & 651,390 & $\$$ & 651,390 & $\$$ & 651,390 & $\$ \quad 651,390$ \\
\hline 12 & $\$$ & 664,418 & $\$$ & 664,418 & $\$$ & 664,418 & $\$ \quad 664,418$ \\
\hline 13 & $\$$ & 677,706 & $\$$ & 677,706 & $\$$ & 677,706 & $\$ \quad 677,706$ \\
\hline 14 & $\$$ & 691,260 & $\$$ & 691,260 & $\$$ & 691,260 & $\$ \quad 691,260$ \\
\hline 15 & $\$$ & 705,085 & $\$$ & 705,085 & $\$$ & 705,085 & $\$ \quad 705,085$ \\
\hline Net Present Costs & $\$$ & $(4,000,000)$ & $\$$ & $(4,000,000)$ & $\$$ & $(4,000,000)$ & $\$(4,000,000)$ \\
\hline Net Present Benefit & $\$$ & $6,280,844$ & $\$$ & $5,474,002$ & $\$$ & $4,813,177$ & $\$ 4,267,260$ \\
\hline NPV & $\$$ & $2,280,844$ & $\$$ & $1,474,002$ & $\$$ & 813,177 & $\$ \quad 267,260$ \\
\hline BCR & & 1.57 & & 1.37 & & 1.20 & 1.07 \\
\hline IRR & & $12 \%$ & & & & & \\
\hline
\end{tabular}

The other NPV analysis for the averaged simulation results, though more conservative, also returned positive values, however only for the MARR values less than $8 \%$. The NPV of the project ranged between $\$ 988,747$ and $-\$ 610,602$ over the same range of MARR values between 
$5 \%$ and $11 \%$. This corresponded to a benefit cost ratio (BCR) between 1.25 and 0.85 . These results are detailed below in Table 6 .

In these scenarios the IRR values were found to be $12 \%$ and $8 \%$, meaning that any MARR value up to and including $12 \%$ for the primary model, or $8 \%$ for the averaged models, would result in the project being deemed attractive and should be accepted. Since the project will be managed by some type of government entity with a lower MARR than a private institution, this is a very attractive IRR.

Table 6: NPV and IRR Analysis of Shared Space Models Average Across All Six Models

\begin{tabular}{|c|c|c|c|c|}
\hline MARR & $5 \%$ & $7 \%$ & $9 \%$ & $11 \%$ \\
\hline Year & Value & Value & Value & Value \\
\hline 0 & $\$(4,000,000)$ & $\$(4,000,000)$ & $\$(4,000,000)$ & $\$(4,000,000)$ \\
\hline 1 & 424,437 & 424,437 & 424,437 & $\$ \quad 424,437$ \\
\hline 2 & 432,925 & 432,925 & 432,925 & $\$ \quad 432,925$ \\
\hline 3 & 441,584 & 441,584 & 441,584 & $\$ \quad 441,584$ \\
\hline 4 & 450,416 & 450,416 & 450,416 & $\$ \quad 450,416$ \\
\hline 5 & 459,424 & 459,424 & 459,424 & $\$ \quad 459,424$ \\
\hline 6 & 468,612 & 468,612 & 468,612 & $\$ \quad 468,612$ \\
\hline 7 & 477,985 & 477,985 & 477,985 & $\$ \quad 477,985$ \\
\hline 8 & 487,544 & 487,544 & 487,544 & 487,544 \\
\hline 9 & 497,295 & 497,295 & 497,295 & $\$ \quad 497,295$ \\
\hline 10 & 507,241 & 507,241 & 507,241 & $\$ \quad 507,241$ \\
\hline 11 & 517,386 & 517,386 & 517,386 & $\$ \quad 517,386$ \\
\hline 12 & 527,734 & 527,734 & 527,734 & 527,734 \\
\hline 13 & 538,288 & 538,288 & 538,288 & $\$ \quad 538,288$ \\
\hline 14 & 549,054 & 549,054 & 549,054 & 549,054 \\
\hline 15 & 560,035 & 560,035 & 560,035 & $\$ \quad 560,035$ \\
\hline Net Present Costs & $\$(4,000,000)$ & $\$(4,000,000)$ & $\$(4,000,000)$ & $\$(4,000,000)$ \\
\hline Net Present Benefit & $\$ 4,988,747$ & $\$ \quad 4,347,889$ & $\$ 3,823,009$ & $\$ 3,389,398$ \\
\hline NPV & 988,747 & 347,889 & $\$ \quad(176,991)$ & $\$(610,602)$ \\
\hline BCR & 1.25 & 1.09 & 0.96 & 0.85 \\
\hline IRR & $8 \%$ & & & \\
\hline
\end{tabular}




\subsection{Discussion}

By combining the positive results of the travel time study, the even more attractive results for the delay analysis, convincing statistics for the queue length observations, and finally the financial feasibility of the project in an economic sense, it was made certain that for this case study location, a shared space design would drastically improve the traffic congestion. Certainly, for other case locations metrics and performance will all change depending on the relative volumes of vehicles and pedestrians. This methodology however could be replicated to analyze such scenarios. 


\section{CONCLUSIONS AND RECOMMENDATIONS}

This study has successfully shown how simulation software such as PTV Vissim can be utilized to simulate and analyze shared space as a proposed solution for traffic congestion problems. By taking advantage of built in functionalities within the existing PTV software package, the planner or engineer, can replicate these efforts to assess unique shared space designs. Due to the simplicity of the methodology and lack of need for additional software training or purposes, we believe this knowledge can be used to assist corporations, government entities, individual planners and engineers, and effectively in return the general citizens and users of this infrastructure.

For the case study location the analysis of the observed simulation data allowed us to see the impacts shared space will have on the selected location. By comparing the shared space results to the simulated current scenario results we were able to quantify the improvements in terms of travel time, delay, queue length, and impact on upstream and downstream intersections. It was observed that average across the six shared space models, the travel time improvements were estimated to be modest. When turning attention to the delay and queue length measurements however we could see a much more drastic improvement. Perhaps most important to be noted from this study was the reduced impact on the intersections at North High Street and Stewart Street which were upstream and downstream of the case study location. At those two intersections it was observed that by implementing a shared space design they would be impacted significantly less from traffic congestion within the case study location. We can then project this information to the larger network as a whole and know that by improving this one location, and observing improvements at two additional locations, the entire network will be incrementally improved. 
Finally, the financial analysis of the proposed project showed how the investment of $\$ 4,000,000$ could leverage increased benefits within the city. Recall that this financial analysis only included the very objective benefit of travel time savings within the shared space and at the two surrounding intersections. The subjective benefits were not included and additional travel time savings between the shared space and these two intersections as well as additional travel time savings around the network were not included. Still, the results were very positive and indicated the project would be very attractive to the government entity that would lead and finance this project.

Overall, a conservative approach was taken to analyze the abilities of shared space at this study location. Therefore it is with higher certainty that we can say it will work to improve traffic congestion at this location. It is our recommendation that shared space be implemented as a solution on the West Virginia University Downtown campus to combat the increasing traffic congestion problem. It is my personal hope that this methodology to evaluate shared space be applied to other case locations around the US to evaluate shared space as an alternative solution to their congestion concerns as well. For future research, it is recommended that the safe navigation of shared spaces by users with disabilities, the legal impacts of shared space, as well as autonomous vehicle navigation of shared space be researched in the future. 


\section{REFERENCES}

“Access.” (1998-2016). The Royal Borough of Kensington and Chelsea. 〈https://www.rbkc.gov.uk/subsites/exhibitionroad/whatschanging/access/quotes.aspx> (Feb. 10, 2016).

Arancibia, D. (2013) Cyclists, Bike Lanes and On-Street Parking: Economic Impacts. Toronto Cyling Think \& Do Tank, Toronto, Canada.

Behrens, G. (2014). "Sharing the Street: Shared Space in an American Context." University of Washington, Urban Planning.

Cabe Space. (2005) "What are we scared of? The value of risk in designing public space." < http://webarchive.nationalarchives.gov.uk/20110118095356/http:/www.cabe.org.uk/files/ what-are-we-scared-of.pdf> (March 22, 2016)

Diosdado-De-La-Pena, M. (2008) "Safety Externalities of SUVs on Passenger Cars: An Analysis of the Peltzman Effect Using FARS Data." West Virginia University College of Engineering and Mineral Resources.

Express, M. (2013). "Traders share in success of Poynton shared space scheme." Macclesfield Express, <http://www.macclesfield-express.co.uk/news/local-news/traders-share-successpoynton-shared-2526261> (Feb. 10, 2016).

Fischer, W. (2011). "Shared Space in Graz (Austria)". Eltis, <http://www.eltis.org/discover/news/shared-space-graz-austria-0> (Feb. 19, 2016).

Garman, F., Luca, O., Singureanu, O. (2012) "Coping with Congestion: Shared Spaces." Theoretical and Empirical Researches in Urban Management, 7(4), 53-62.

Gibb, S. (2015). Simulating The Streets of Tomorrow. CH2M, Bristol, UK. Issue 2.

Gilman, C., Gilman, R. (2007) "Shared-Use Streets - An Application of "Shared Space" to an American Small Town." $3^{\text {rd }}$ Urban Street Symposium, Seattle, WA.

Gillies, A. (2009). "Is the road there to share? Shared space in an Australian context." University of New South Wales, Bachelor of Planning.

Hall, A. (2008). "Accident-free zone: The German town which scrapped all traffic lights and road signs." Daily Mail, <http://www.dailymail.co.uk/news/article-1028740/Accidentfree-zone-The-German-town-scrapped-traffic-lights-road-signs.html> (Feb. 10, 2016).

Hamilton-Baillie, B. (2008). "Shared Space: Reconciling People, Places and Traffic." Built Environment (1978), 34(2), 161-181.

Hamilton-Baillie, B. (2008). “Towards shared space.” Urban Design International, 13, 130-138. 
Hawkins, H. G. Jr. PhD, PE. (2015) “The MUTCD Turns 80: Time for a Makeover." Institute of Transportation Engineers. ITE Journal, 85(11), 14-16.

James, K. (2008) “German Town's Traffic Plan: Remove Signs, Curbs.” National Public Radio, $<$ http://www.npr.org/templates/story/story.php?storyId=18217318> (Feb. 10, 2016).

Kaparias, I., Bell, M. G. H., Biagioli, T., Bellezza, L., and Mount, B. (2015). "Behavioural analysis of interactions between pedestrians and vehicles in street designs with elements of shared space.” Transportation Research, F(30), 115-127.

Kaparias, I., Bell, M. G. H., Dong, W., Sastrawinata, A., Singh, A., Wang, X., and Mount, B. (2013). "Analysis of Pedestrian-Vehicle Traffic Conflicts in Street Designs with Elements of Shared Space." Transportation Research Record: Journal of the Transportation Research Board, 2393, 21-30.

Kaparias, I., Bell, M. G. H., Miri, A., Chan, C., and Mount, B. (2012). "Analysing the perceptions of pedestrians and drivers to shared space." Transportation Research, $\mathrm{F}(15)$, 297-310.

Kirkup, A. (2013) "Poynton town centre." Institution of Civil Engineers, <https://www.ice.org.uk/disciplines-and-resources/case-studies/poynton-town-centre> (Feb. 10, 2016)

Langdon, P. (2010). "US shared space: Starting small.” Better Cities \& Towns. < http://bettercities.net/article/us-shared-space-starting-small-13673> (Feb. 19, 2016).

Leaf, W. A., and Preusser, D. F. (1999). "Literature Review on Vehicle Travel Speeds and Pedestrian Injuries Among Selected Racial/Ethnic Groups." U. S. Department of Transportation: National Highway Traffic Safety Administration, Washington, D. C.

MMMPO. (2016) “2016 Traffic Count Report (Draft).” <https://drive.google.com/file/d/0B8UM6dsVoQTINVYyUE1qeUp0dzA/view> (May 26, 2017)

Pascucci, F., Rinke, N., Schiermeyer, C., Friedrich, B., Berkhahn, V. (2015) "Modeling of shared space with multi-model traffic using a multi-layer social force approach." Transportation Research Procedia. 10. 316-326.

Resource Systems Group, Inc. (2012) Economic Impact of Bicycling and Walking in Vermont. Vermont Agency of Transportation, Montpelier, VT.

Rudloff, C., Schonauer, R., Fellendorf, M. (2013). "Comparing Calibrated Shared Space Simulation Model with Real-Life Data." Transportation Research Record. 2390. 44-52.

Schmidt, A.(2016) "Study: 'Shared Space' Slows Drivers While Letting Traffic Move Efficiently." Streetsblog USA. <http://usa.streetsblog.org/2016/02/08/study-shared-spaceslows-drivers-but-speeds-up-traffic/> (Feb. 19, 2016) 
Schonauer, R., Stubenschrott, M., Huang, W., Rudloff, C., Fellendorf, M. (2012). "Modeling Concepts for Mixed Traffic." Transportation Research Record. 2316. 114-121.

"Shared Space Implementation Sonnenfelsplatz in Graz, Austria". (2011). Recodrive. <www.recodrive.eu/PDF/generate_pdf.php?study_id=3504\&lan=en>

Shore, F., Uthayakumar, K. (2010) Shared Space: Operational Assessment, MVA Consultancy, 17 Hanover Square, London, UK.

Snyder, T. (2014). "Bikes, Cars, and People Co-Exist on Pittsburgh's Shared Streets." Streetsblog USA. < http://usa.streetsblog.org/2014/07/03/bikes-cars-and-people-co-existon-pittsburghs-shared-streets/> (Feb. 19, 2016).

Schmitz, J. (2015) "Experts say shared space, transportation free-for-all makes sense for Pittsburgh." Pittsburgh Post-Gazette.

Tawil, M., Reicher, C., Ramadan, K. Z., Jafari, M. (2014) “Towards More Pedestrian Friendly Streets in Jordan: The Case of al Medina Street in Amman." Journal of Sustainable Development. 7(2), 144-158.

"Texas A\&M University Reports Findings in Transportation Policy (Investigating Peltzman effects in adopting mandatory seat belt laws in the US: Evidence from non-occupant fatalities)." (2015) Journal of Transportation. 197

US Census Bureau. (2016) "Quick Facts." <https://www.census.gov/quickfacts/table/PST045216/00> (April 26, 2017).

Whitlock, C. (2007) “A Green Light for Common Sense.” The Washington Post Foreign Service, $<$ http://www.washingtonpost.com/wpdyn/content/article/2007/12/23/AR2007122302487.html> (Feb. 10, 2016).

"Why making streets risky improves road safety." (2012).Allianz. <https://www.allianz.com/en/about_us/open-knowledge/topics/mobility/articles/120416why-making-streets-risky-improves-road-safety.html/> (Feb. 19, 2016).

Wilkinson, M. (2014) "Britain's town centre roads need more shared spaces, expert says." The Telegraph, <http://www.telegraph.co.uk/motoring/road-safety/11072664/Britains-towncentre-roads-need-more-shared-spaces-expert-says.html> (Feb. 10, 2016).

Yoe, C. (2011) "Primer on Risk Analysis Decision Making Under Uncertainty." CRC Press. 113-153. 


\section{APPENDIX}

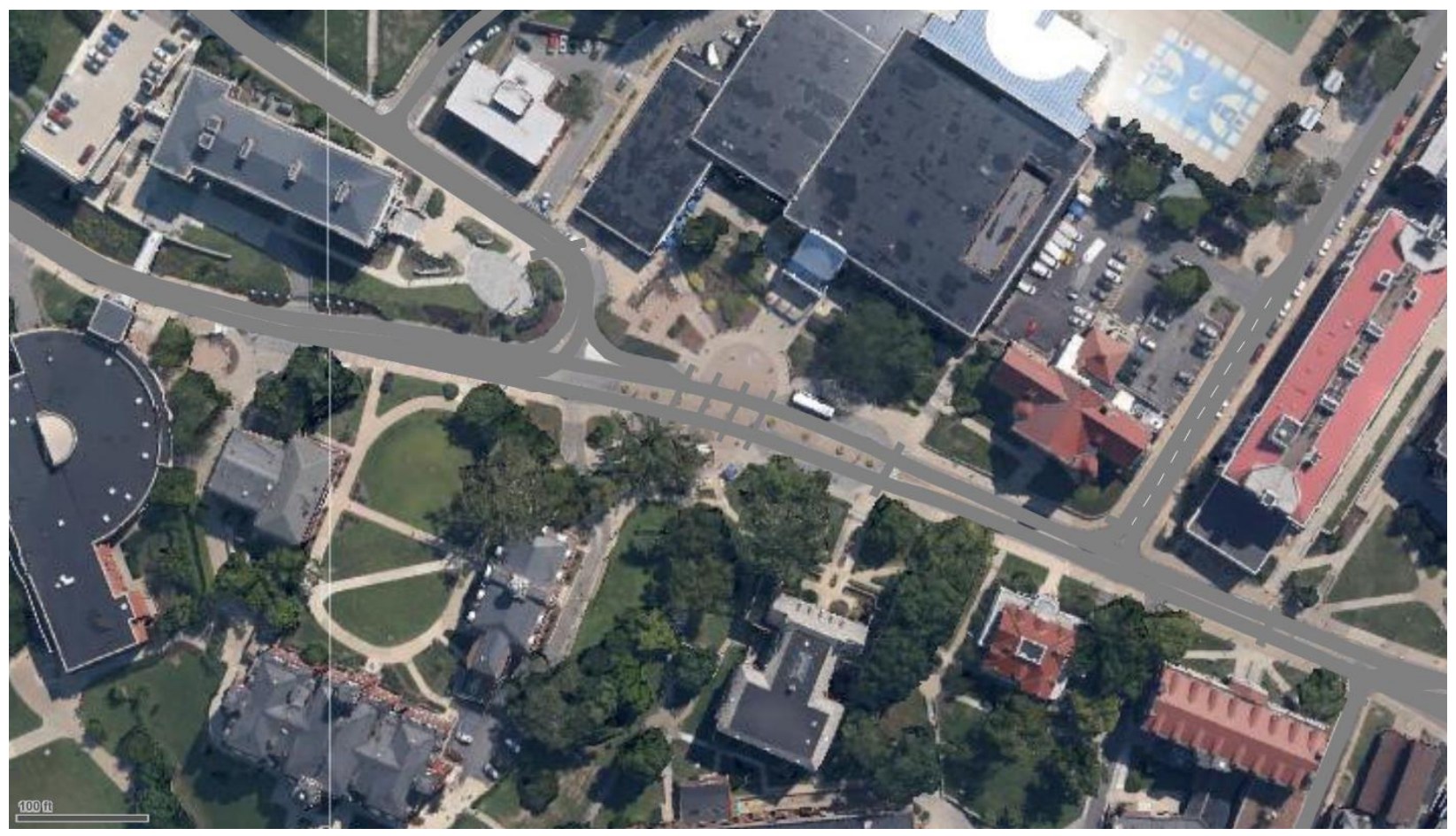

Figure 44: Pedestrian Crossing and Vehicle Travel Links in PTV Vissim Model of Current State

Table 7: Pedestrian Volumes Used in model of Current Traffic

\begin{tabular}{|c|c|c|c|c|c|c|}
\hline & From & To & $0-900$ & $900-1800$ & $1800-2700$ & $2700-3600$ \\
\hline \multirow{13}{*}{ 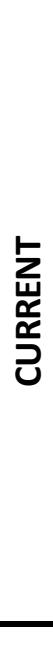 } & Clark & Stewart & 100 & 200 & 100 & 100 \\
\hline & Clark & Colson & 100 & 200 & 100 & 100 \\
\hline & Colson & St John & 100 & 200 & 100 & 100 \\
\hline & Colson & Clark & 100 & 200 & 100 & 100 \\
\hline & E Moore & Stewart & 100 & 200 & 100 & 100 \\
\hline & Lair & PRT & 800 & 1600 & 500 & 500 \\
\hline & Lair & Oglebay & 300 & 600 & 200 & 200 \\
\hline & Oglebay & Lair & 300 & 600 & 200 & 200 \\
\hline & PRT & Lair & 800 & 1600 & 500 & 500 \\
\hline & St John & Colson & 100 & 200 & 100 & 100 \\
\hline & Stewart & E Moore & 100 & 200 & 100 & 100 \\
\hline & Stewart & Clark & 100 & 200 & 100 & 100 \\
\hline & \multicolumn{2}{|c|}{ Total } & 3000 & 6000 & 2200 & 2200 \\
\hline
\end{tabular}


Table 8: Pedestrian Volumes Used in Model of Shared Space

\begin{tabular}{|c|c|c|c|c|c|c|c|c|c|c|c|c|c|c|c|c|}
\hline From & To & \multirow{35}{*}{ 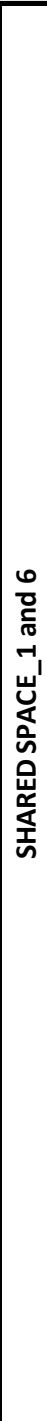 } & $0-900$ & $900-1800$ & $1800-2700$ & $2700-3600$ & \multirow{35}{*}{ 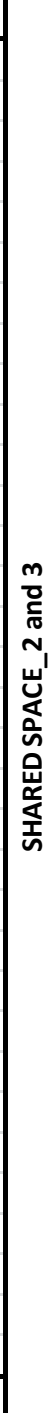 } & $0-900$ & $900-1800$ & $1800-2700$ & 2700-3600 & \multirow{35}{*}{ 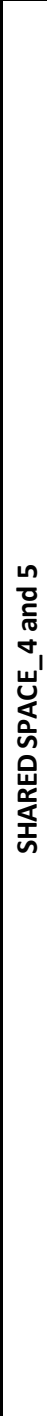 } & $0-900$ & $900-1800$ & $1800-2700$ & $2700-3600$ \\
\hline$B \& E$ & Oglebay & & 25 & 50 & 25 & 25 & & 95 & 190 & 75 & 75 & & 25 & 50 & 25 & 25 \\
\hline Clark & Stewart & & 75 & 150 & 65 & 65 & & 95 & 190 & 75 & 75 & & 75 & 150 & 50 & 50 \\
\hline Colson & Library & & 75 & 150 & 65 & 65 & & 95 & 190 & 75 & 75 & & 75 & 150 & 50 & 50 \\
\hline Colson & Library & & 25 & 50 & 25 & 25 & & 95 & 190 & 75 & 75 & & 25 & 50 & 25 & 25 \\
\hline Colson & St John/Library & & 125 & 250 & 100 & 100 & & 95 & 190 & 75 & 75 & & 100 & 200 & 75 & 75 \\
\hline E Moore & Lair-R & & 50 & 100 & 50 & 50 & & 95 & 190 & 75 & 75 & & 25 & 50 & 25 & 25 \\
\hline E Moore & Lair-R & & 50 & 100 & 50 & 50 & & 95 & 190 & 75 & 75 & & 25 & 50 & 25 & 25 \\
\hline E Moore & Stewart & & 25 & 50 & 25 & 25 & & 95 & 190 & 75 & 75 & & 25 & 50 & 25 & 25 \\
\hline Lair-C & PRT-R & & 250 & 500 & 150 & 150 & & 95 & 190 & 75 & 75 & & 275 & 625 & 225 & 225 \\
\hline Lair-C & Woodburn/Oglebay & & 250 & 500 & 150 & 150 & & 95 & 190 & 75 & 75 & & 275 & 625 & 225 & 225 \\
\hline Lair-L & Oglebay & & 75 & 150 & 65 & 65 & & 95 & 190 & 75 & 75 & & 150 & 250 & 75 & 75 \\
\hline Lair-L & Oglebay & & 225 & 450 & 150 & 150 & & 95 & 190 & 75 & 75 & & 150 & 250 & 75 & 75 \\
\hline Lair-L & Oglebay & & 200 & 400 & 125 & 125 & & 95 & 190 & 75 & 75 & & 75 & 150 & 75 & 75 \\
\hline Lair-R & E Moore & & 75 & 150 & 65 & 65 & & 95 & 190 & 75 & 75 & & 100 & 200 & 50 & 50 \\
\hline Lair-R & E Moore & & 200 & 400 & 125 & 125 & & 95 & 190 & 75 & 75 & & 100 & 200 & 50 & 50 \\
\hline Library & Colson & & 100 & 200 & 80 & 80 & & 95 & 190 & 75 & 75 & & 100 & 200 & 80 & 80 \\
\hline Oglebay & $B \& E$ & & 25 & 50 & 25 & 25 & & 95 & 190 & 75 & 75 & & 25 & 50 & 25 & 25 \\
\hline Oglebay & Lair & & 75 & 150 & 65 & 65 & & 95 & 190 & 75 & 75 & & 50 & 100 & 50 & 50 \\
\hline Oglebay & Lair-L & & 75 & 150 & 65 & 65 & & 95 & 190 & 75 & 75 & & 50 & 100 & 50 & 50 \\
\hline Oglebay & Woodburn & & 75 & 150 & 65 & 65 & & 95 & 190 & 75 & 75 & & 100 & 200 & 75 & 75 \\
\hline Oglebay & Woodburn & & 25 & 50 & 25 & 25 & & 95 & 190 & 75 & 75 & & 25 & 50 & 25 & 25 \\
\hline Oglebay & Woodburn/Lair-L & & 75 & 150 & 65 & 65 & & 95 & 190 & 75 & 75 & & 150 & 250 & 100 & 100 \\
\hline PRT-C & Lair-C & & 150 & 300 & 100 & 100 & & 95 & 190 & 75 & 75 & & 300 & 625 & 250 & 250 \\
\hline PRT-L & Lair-C & & 150 & 300 & 100 & 100 & & 95 & 190 & 75 & 75 & & 275 & 625 & 225 & 225 \\
\hline PRT-L & Oglebay/Lair-L & & 150 & 300 & 100 & 100 & & 95 & 190 & 75 & 75 & & 150 & 250 & 100 & 100 \\
\hline PRT-R & Lair-C & & 100 & 200 & 80 & 80 & & 95 & 190 & 75 & 75 & & 150 & 250 & 100 & 100 \\
\hline Purinton & Stewart & & 25 & 50 & 25 & 25 & & 95 & 190 & 75 & 75 & & 25 & 50 & 25 & 25 \\
\hline St John & Colson & & 100 & 200 & 80 & 80 & & 95 & 190 & 75 & 75 & & 50 & 100 & 25 & 25 \\
\hline Stewart & Clark/Purinton & & 75 & 150 & 65 & 65 & & 95 & 190 & 75 & 75 & & 75 & 150 & 50 & 50 \\
\hline Stewart & E Moore & & 25 & 50 & 25 & 25 & & 95 & 190 & 75 & 75 & & 25 & 50 & 25 & 25 \\
\hline Woodburn & Oglebay & & 50 & 100 & 50 & 50 & & 95 & 190 & 75 & 75 & & 25 & 50 & 25 & 25 \\
\hline Woodburn & Oglebay & & 25 & 50 & 25 & 25 & & 95 & 190 & 75 & 75 & & 25 & 50 & 25 & 25 \\
\hline Woodburn & Oglebay/Lair-L & & 100 & 200 & 80 & 80 & & 95 & 190 & 75 & 75 & & 25 & 50 & 25 & 25 \\
\hline & Total & & 3125 & 6250 & 2355 & 2355 & & 3135 & 6270 & 2475 & 2475 & & 3125 & 6250 & 2355 & 2355 \\
\hline
\end{tabular}

